quatrième série-tome $46 \quad$ fascicule 2 mars-avril 2013

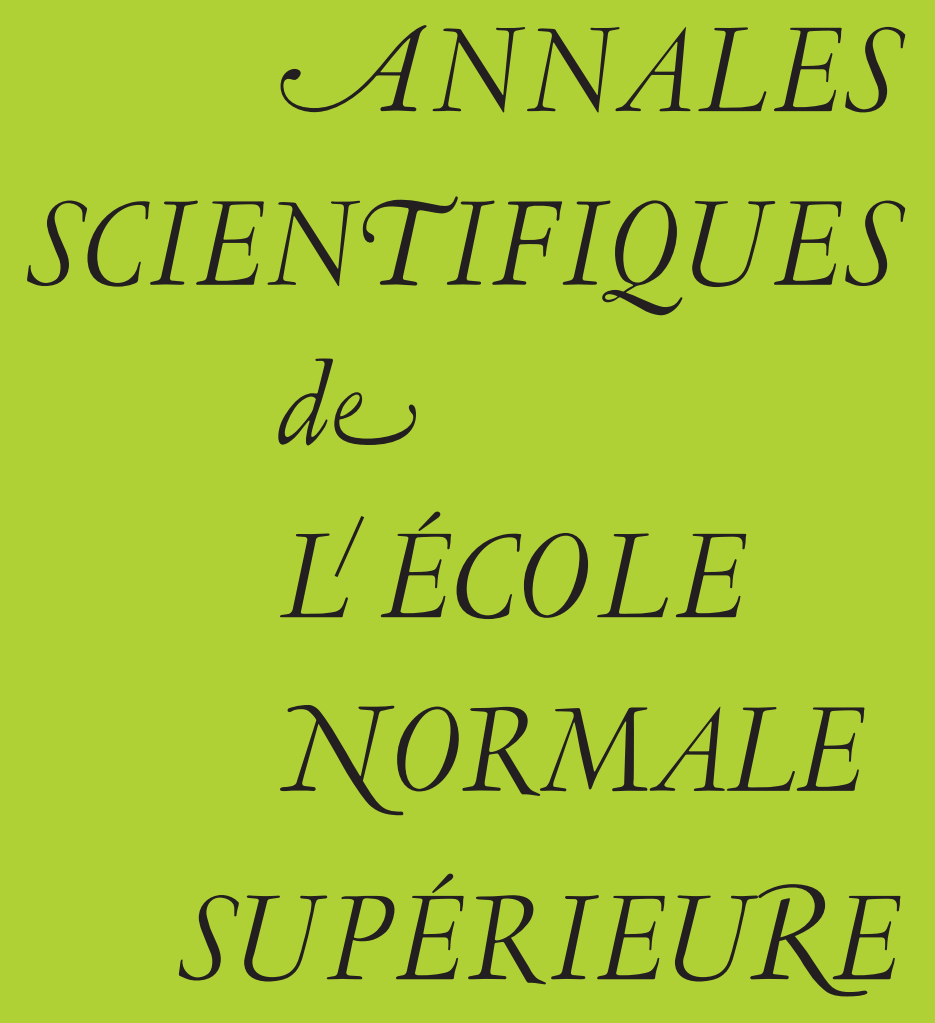

Nikolay TZVETKOV \& Nicola VISCIGLIA

Gaussian measures associated to the bigher order conservation laws of the Benjamin-Ono equation 
Ann. Scient. Éc. Norm. Sup.

$4^{\text {e }}$ série, t. 46, 2013, p. 249 à 299

\title{
GAUSSIAN MEASURES ASSOCIATED TO THE HIGHER ORDER CONSERVATION LAWS OF THE BENJAMIN-ONO EQUATION
}

\author{
BY Nikolay TZVETKOV and Nicola VISCIGLIA
}

\begin{abstract}
Inspired by the work of Zhidkov on the KdV equation, we perform a construction of weighted Gaussian measures associated to the higher order conservation laws of the BenjaminOno equation. The resulting measures are supported by Sobolev spaces of increasing regularity. We also prove a property on the support of these measures leading to the conjecture that they are indeed invariant by the flow of the Benjamin-Ono equation.

RÉSUMÉ. - Inspirés par le travail de Zhidkov sur l'équation $\mathrm{KdV}$, nous construisons des mesures gaussiennes à poids associées à une loi de conservation arbitraire de l'équation de Benjamin-Ono. Les supports de ces mesures sont constitués de fonctions de régularité de Sobolev croissantes. On démontre aussi une propriété-clé des mesures qui nous conduit à conjecturer leur invariance par le flot de l'équation.
\end{abstract}

\section{Introduction and statement of the results}

\subsection{Measures construction}

The main goal of this article is to construct weighted Gaussian measures associated with an arbitrary conservation law of the Benjamin-Ono equation (BO), and thus to extend the result of the first author [14] which deals only with the first conservation law. The analysis contains several significant elaborations with respect to [14]; it requires an understanding of the interplay between the structure of the conservation laws of the Benjamin-Ono equation and the probabilistic arguments involved in the renormalization procedure defining the measures.

Let us recall that just like the $\mathrm{KdV}$ equation, the Benjamin-Ono equation is a basic dispersive PDE describing the propagation of one directional, long, small amplitude waves. The difference between the $\mathrm{KdV}$ and $\mathrm{BO}$ equations is that the $\mathrm{KdV}$ equation describes surface waves while the Benjamin-Ono equation models the propagation of internal waves. These models have rich mathematical structure from both the algebraic and analytical viewpoints. 
In particular they have an infinite sequence of conservation laws. These aspects will be heavily exploited in the present work.

Consider now the Benjamin-Ono equation

$$
\partial_{t} u+H \partial_{x}^{2} u+u \partial_{x} u=0
$$

with periodic boundary conditions (for simplicity throughout the paper we fix the period to be equal to $2 \pi$ ). In (1.1), $H$ denotes the Hilbert transform acting on periodic distributions. Thanks to the work of Molinet [11] (1.1) is globally well-posed in $H^{s}, s \geq 0$ (see [13, 7, 5] for related results in the case when (1.1) is posed on the real line).

It is well-known that (smooth) solutions to (1.1) satisfy an infinite number of conservation laws (see e.g., $[10,1])$. More precisely for $k \geq 0$ an integer, there is a conservation law of (1.1) of the form

$$
E_{k / 2}(u)=\|u\|_{\dot{H}^{k / 2}}^{2}+R_{k / 2}(u)
$$

where $\dot{H}^{s}$ denotes the homogeneous Sobolev norm on periodic functions, and all the terms that appear in $R_{k / 2}$ are homogeneous of the order larger than or equal to three in $u$. In Section 2, we will describe in more details the structure of $R_{k / 2}$ for large $k$. Next we explicitly write the conservation laws $E_{k / 2}$ for $k=0,1,2,3,4$ :

$$
\begin{aligned}
E_{0}(u)= & \|u\|_{L^{2}}^{2} ; \\
E_{1 / 2}(u)= & \|u\|_{\dot{H}^{1 / 2}}^{2}+\frac{1}{3} \int u^{3} d x ; \\
E_{1}(u)= & \|u\|_{\dot{H}^{1}}^{2}+\frac{3}{4} \int u^{2} H\left(u_{x}\right) d x+\frac{1}{8} \int u^{4} d x \\
E_{3 / 2}(u)= & \|u\|_{\dot{H}^{3 / 2}}^{2}-\int\left[\frac{3}{2} u\left(u_{x}\right)^{2}+\frac{1}{2} u H\left(u_{x}\right)^{2}\right] d x \\
& -\int\left[\frac{1}{3} u^{3} H\left(u_{x}\right)+\frac{1}{4} u^{2} H\left(u u_{x}\right)\right] d x-\frac{1}{20} \int u^{5} d x ; \\
E_{2}(u)= & \|u\|_{\dot{H}^{2}}^{2}-\frac{5}{4} \int\left[\left(u_{x}\right)^{2} H u_{x}+2 u u_{x x} H u_{x}\right] d x \\
& +\frac{5}{16} \int\left[5 u^{2}\left(u_{x}\right)^{2}+u^{2} H\left(u_{x}\right)^{2}+2 u H\left(\partial_{x} u\right) H\left(u u_{x}\right)\right] d x \\
& +\int\left[\frac{5}{32} u^{4} H\left(u_{x}\right)+\frac{5}{24} u^{3} H\left(u u_{x}\right)\right] d x+\frac{1}{48} \int u^{6} d x
\end{aligned}
$$

where $\int$ is understood as the integral on the period $(0,2 \pi)$.

Following the work by Zhidkov [15] (see also [2, 8]), one may try to define an invariant measure for (1.1) by re-normalizing the formal measure $\exp \left(-E_{k / 2}(u)\right) d u$. This re-normalization is a delicate procedure. One possibility would be first to re-normalize $\exp \left(-\|u\|_{\dot{H}^{k / 2}}^{2}\right) d u$ as a Gaussian measure on an infinite dimensional space and then to show that the factor $\exp \left(-R_{k / 2}(u)\right)$ is integrable with respect to this measure.

Since $\exp \left(-\|u\|_{\dot{H}^{k / 2}}^{2}\right)$ factorizes as an infinite product when we express $u$ as a Fourier series, we can define the re-normalization of $\exp \left(-\|u\|_{\dot{H}^{k / 2}}^{2}\right) d u$ as the Gaussian measure 
induced by the random Fourier series

$$
\varphi_{k / 2}(x, \omega)=\sum_{n \neq 0} \frac{\varphi_{n}(\omega)}{|n|^{k / 2}} e^{\mathrm{i} n x}
$$

(one may ignore the zero Fourier mode since the mean of $u$ is conserved by the flow of (1.1)). In (1.3), $\left(\varphi_{n}(\omega)\right)_{n \neq 0}$ is a sequence of standard complex Gaussian variables defined on a probability space $(\Omega, \mathscr{G}, p)$ such that $\varphi_{n}=\overline{\varphi_{-n}}$ (since the solutions of (1.1) should be real valued) and $\left(\varphi_{n}(\omega)\right)_{n>0}$ are independent. Let us denote by $\mu_{k / 2}$ the measure induced by (1.3). One may easily check that $\mu_{k / 2}\left(H^{s}\right)=1$ for every $s<(k-1) / 2$ while $\mu_{k / 2}\left(H^{(k-1) / 2}\right)=0$.

In view of the previous discussion, one may consider $\exp \left(-R_{k / 2}(u)\right) d \mu_{k / 2}$ as a candidate of invariant measure for (1.1). There are two obstructions to do that, the first one already appears in previous works on the NLS equation (see [2, 8]) and the KdV equation (see [15]), while the second one is specific to the Benjamin-Ono equation. The first obstruction is that $\exp \left(-R_{k / 2}(u)\right)$ is not integrable with respect to $d \mu_{k / 2}(u)$. This problem may be resolved by restricting to invariant sets, which means to replace $\exp \left(-R_{k / 2}(u)\right)$ by

$$
\prod_{j=0}^{k-1} \chi_{R}\left(E_{j / 2}(u)\right) e^{-R_{k / 2}(u)}
$$

where $\chi_{R}$ is a cut-off function defined as $\chi_{R}(x)=\chi(x / R)$ with $\chi: \mathbb{R} \rightarrow \mathbb{R}$ a continuous, compactly supported function such that $\chi(x)=1$ for every $|x|<1$. In the context of $\mathrm{KdV}$ or NLS, the function defined in (1.4) is integrable with respect to the corresponding Gaussian measure. Moreover if one takes the reunion over $R>0$ of the supports of the functions (1.4), then one obtains a set containing the support of $\mu_{k / 2}$. However, in the context of the Benjamin-Ono equation, the restriction to invariant sets does not work as in (1.4) because for every $R$ the following occurs: $\chi_{R}\left(E_{(k-1) / 2}(u)\right)=0$ almost surely on the support of $\mu_{k / 2}$. One of the main points of this paper is to resolve this difficulty. This will be possible since one controls the way that $E_{(k-1) / 2}(u)$ diverges on the support of $\mu_{k / 2}$. More precisely, for $N \geq 1$ and $k \geq 2$, we introduce the function

$$
F_{k / 2, N, R}(u)=\left(\prod_{j=0}^{k-2} \chi_{R}\left(E_{j / 2}\left(\pi_{N} u\right)\right)\right) \chi_{R}\left(E_{(k-1) / 2}\left(\pi_{N} u\right)-\alpha_{N}\right) e^{-R_{k / 2}\left(\pi_{N} u\right)}
$$

where $\alpha_{N}=\sum_{n=1}^{N} \frac{1}{n}$ and $\pi_{N}$ is the Dirichlet projector on Fourier modes $n$ such that $|n| \leq N$. Here is our first result.

Theorem 1.1. - For every $k \in \mathbb{N}$ with $k \geq 2$, there exists a $\mu_{k / 2}$ measurable function $F_{k / 2, R}(u)$ such that $F_{k / 2, N, R}(u)$ converges to $F_{k / 2, R}(u)$ in $L^{q}\left(d \mu_{k / 2}\right)$ for every $1 \leq q<\infty$. In particular $F_{k / 2, R}(u) \in L^{q}\left(d \mu_{k / 2}\right)$. Moreover, if we set $d \rho_{k / 2, R} \equiv F_{k / 2, R}(u) d \mu_{k / 2}$, we have

$$
\bigcup_{R>0} \operatorname{supp}\left(\rho_{k / 2, R}\right)=\operatorname{supp}\left(\mu_{k / 2}\right) \text {. }
$$

The above result for $k=1$ was obtained by the first author in [14]. Many of the probabilistic techniques involved in the proof of Theorem 1.1 are inspired by [3]. We also refer to [4] where in the context of the $2 d$ NLS the authors use the Wick ordered $L^{2}$-cutoff, i.e., a truncation of the $L^{2}$-norm that depends on the parameter $N$. 
We conjecture that the measures $\rho_{k / 2, R}, k=2,3, \cdots$ constructed in Theorem 1.1 are invariant by the flow of the Benjamin-Ono equation established by Molinet [11], at least for even values of $k$. In the sequel, for shortness, we denote $\rho_{k / 2, R}$ by $\rho_{k / 2}$.

\subsection{A property on the support of the measures}

Let us now give our argument in support of the above-stated conjecture. For $N \geq 1$, we introduce the truncated Benjamin-Ono equation:

$$
\partial_{t} u+H \partial_{x}^{2} u+\pi_{N}\left(\left(\pi_{N} u\right) \partial_{x}\left(\pi_{N} u\right)\right)=0 .
$$

As in [6], one can define a global solution of (1.6) for every initial data $u(0) \in L^{2}\left(S^{1}\right)$. Indeed, one obtains that $\left(1-\pi_{N}\right) u(t)$ is given by the free Benjamin-Ono evolution with data $\left(1-\pi_{N}\right) u(0)$, while $\pi_{N} u(t)$ evolves under an $N$-dimensional ODE. This ODE has a welldefined global dynamics since the $L^{2}$ norm is preserved.

The main problem that appears when one tries to prove the invariance of $\rho_{k / 2}$ is that even if $E_{k / 2}$ are invariants for the Benjamin-Ono equation they are not invariant under (1.6). The invariance, however, holds in a suitable asymptotic sense as we explain below. Let us introduce the real-valued function $G_{k / 2, N}$, measuring the lack of conservation of $E_{k / 2}$ under the truncated flow (1.6), via the following relation

$$
\frac{d}{d t} E_{k / 2}\left(\pi_{N} u(t)\right)=G_{k / 2, N}\left(\pi_{N} u(t)\right)
$$

where $u(t)$ solves (1.6).

Denote by $\Phi_{N}$ the flow of (1.6) and set $d \rho_{N}(u) \equiv F_{k / 2, N, R}(u) d \mu_{k / 2}(u)$ so that by Theorem 1.1, $\rho_{N}$ converges in a strong sense to $\rho_{k / 2}$ (the densities converge in any $L^{p}\left(d \mu_{k / 2}\right)$, $p<\infty)$. By using the Liouville theorem, one shows that for every $\mu_{k / 2}$ measurable set $A$,

$$
\rho_{N}\left(\Phi_{N}(t)(A)\right)=\int_{A} e^{-\int_{0}^{t} G_{k / 2, N}\left(\pi_{N} \Phi_{N}(\tau)(u(0)) d \tau\right.} d \rho_{N}(u(0))+o(1) .
$$

Hence, a main step towards a proof of the invariance of $\rho_{k / 2}$ is to show that

$$
\int_{0}^{t} G_{k / 2, N}\left(\pi_{N} u(\tau)\right) d \tau
$$

converges to zero, where $u(\tau)$ is a solution of (1.6), with $u(0)$ on the support of $\mu_{k / 2}$. Such a property is relatively easy to be established if $u(0)$ has slightly more regularity than the typical Sobolev regularity on the support of $\mu_{k / 2}$. At the present moment, we are not able to prove such a property on the support of $\mu_{k / 2}$. We shall, however, prove it if we make a first approximation which consists of replacing $u(\tau)$ by $u(0)$ in (1.8). Here is the precise statement.

TheOREM 1.2. - For every $k \geq 6$ an even integer, we have

$$
\lim _{N \rightarrow \infty}\left\|G_{k / 2, N}\left(\pi_{N} u\right)\right\|_{L^{q}\left(d \mu_{k / 2}\right)}=0, \forall q \in[1, \infty),
$$

where $G_{k / 2, N}$ is defined by (1.7).

Let us remark that the lack of invariance of conservation laws for the corresponding truncated flows is a problem that appears also in other contexts. We refer in particular to the papers [12] and [15], where this difficulty is resolved in the cases of the DNLS and KdV equations respectively. 


\subsection{Comparison with the $\mathrm{KdV}$ equation}

Next we explain why the measures construction in the context of the Benjamin-Ono equation is much more involved compared with the case of the $\mathrm{KdV}$ equation. The main difference is that in the $\mathrm{KdV}$ equation the dispersion (of the linear part) is of lower order compared with the Benjamin-Ono equation. This fact makes the perturbative treatment of the nonlinearity more complicated. Let us recall that a similar observation applies to the Cauchy problem analysis (see $[5,7,11,13]$ ).

Now, we recall the approach of Zhidkov (see [15]) to prove the existence of invariant measures associated with the periodic $\mathrm{KdV}$ equation

$$
\partial_{t} u+\partial_{x}^{3} u+u \partial_{x} u=0
$$

This equation has a rich structure from both the algebraic and analytic viewpoint. In particular the solutions to (1.9) have an infinite sequence of conversation laws. More precisely, for every $m \geq 0$ there exists a polynomial

$$
p_{m}\left(v, \partial_{x} v, \ldots, \partial_{x}^{m} v\right)
$$

such that $\frac{d}{d t} \int p_{m}\left(u(t, x), \partial_{x} u(t, x), \ldots, \partial_{x}^{m} u(t, x)\right) d x=0$, provided that $u$ is a solution of (1.9), where $\int \cdots d x$ denotes the integral on the period. More precisely, the conservation laws have the following structure

$$
\|u\|_{\dot{H}^{m}}^{2}+\int q_{m}\left(u, . ., \partial_{x}^{m-1} u\right) d x .
$$

By using the Sobolev embedding $H^{1} \subset L^{\infty}$, it is easy to check that the function

$$
\left(\prod_{j=0}^{m-1} \chi_{R}\left(E_{j}(u)\right)\right) e^{-\int q_{m}\left(u, . ., \partial_{x}^{m-1} u\right) d x},
$$

where $\chi_{R}$ is defined as in (1.4), is not trivial and belongs to the space $L^{\infty}\left(d \mu_{m}\right)$ (where $\mu_{m}$ is the Gaussian measure induced by (1.3) for $k=2 m$ ), provided that $m$ is large enough. In particular the measure

$$
\left(\prod_{j=0}^{m-1} \chi_{R}\left(E_{j}(u)\right)\right) e^{-\int q_{m}\left(x, u, . ., \partial_{x}^{m-1} v\right) d x} d \mu_{m}
$$

is a meaningful non-trivial candidate for an invariant measure.

In order to prove the invariance of the above measure we introduce, following [15], a family of truncated problems

$$
\partial_{t} u+\partial_{x}^{3} u+\pi_{N}\left(\left(\pi_{N} u\right) \partial_{x}\left(\pi_{N} u\right)\right)=0
$$

where $\pi_{N}$ is the Dirichlet projector on the $n$ Fourier modes such that $|n| \leq N$. Once again, the main difficulty is because of the fact that the quantity

$$
\int p_{m}\left(u(t, x), \partial_{x} u(t, x), \ldots, \partial_{x}^{m} u(t, x)\right) d x
$$

is no longer invariant along the flow of the truncated problem (1.11). However if $u_{0} \in H^{m-1}$ then

$$
\lim _{N \rightarrow \infty} \frac{d}{d t} \int p_{m}\left(\pi_{N} u(t, x), \partial_{x} \pi_{N} u(t, x), \ldots, \partial_{x}^{m} \pi_{N} u(t, x)\right) d x=0
$$


where $u(t, x)$ are solutions to (1.11) with initial data $u_{0}$. Roughly speaking (1.12) means that the quantities

$$
\int p_{m}\left(\pi_{N} u(t, x), \partial_{x} \pi_{N} u(t, x), \ldots, \partial_{x}^{m} \pi_{N} u(t, x)\right) d x
$$

are asymptotically in $N$ almost conservation laws for solutions to the truncated flow. In particular for large $N$, the classical finite dimensional Liouville invariance theorem turns out to be almost true for the flow associated with (1.11), and it allows us to conclude the proof of the invariance of (1.10) along the flow associated with (1.9) via a limit argument.

Hence the main point is to prove (1.12). Following Zhidkov (see Lemma IV.3.5, page 127 in [15]) there is an explicit formula to compute the expression on the 1.h.s. in (1.12). More precisely if $u$ solves (1.11) then

$$
\begin{aligned}
\frac{d}{d t} \int p_{m}\left(\pi_{N} u(t\right. & \left., x), \partial_{x} \pi_{N} u(t, x), \ldots, \partial_{x}^{m} \pi_{N} u(t, x)\right) d x \\
& =\sum_{j=0}^{m} \int\left(\frac{\partial p_{m}}{\partial_{x}^{j} u}\right)_{\mid \partial_{x}^{j} u=\partial_{x}^{j} \pi_{>N}\left(\left(\pi_{N} u\right) \partial_{x}\left(\pi_{N} u\right)\right), \partial_{x}^{k} u=\partial_{x}^{k} \pi_{N} u \text { for } k \neq j} d x
\end{aligned}
$$

where $\pi_{>N}$ is the projector on the $n$ Fourier modes such that $|n|>N$ (for an explanation of Formula (1.13) see Section 8). It is easy to see that the most delicate term that appears in the r.h.s. above is the one coming from the cubic part of the conservation laws, i.e., $\int u\left(\partial_{x}^{m-1} u\right)^{2} d x$. More precisely we have to estimate the following term

$$
\int\left(\pi_{N} u\right) \partial_{x}^{m-1}\left(\pi_{N} u\right) \partial_{x}^{m-1} \pi_{>N}\left(\left(\pi_{N} u\right) \partial_{x}\left(\pi_{N} u\right)\right) d x,
$$

as $N \rightarrow \infty$. Notice that after developing the $(m-1)$-derivative of the product, we get an integral that involves the product of $\partial_{x}^{m-1}\left(\pi_{N} u\right)$ and $\partial_{x}^{m}\left(\pi_{N} u\right)$, and hence after a fractional integration by parts we get a derivative of order $m-1 / 2$. This is the main source of difficulty since the Gaussian measure $d \mu_{m}$ is supported on the Sobolev spaces $H^{m-1 / 2-\epsilon}$ for any $\epsilon>0$. This problem is solved by Zhidkov by using a clever integration by parts. Indeed, if we develop the $(m-1)$-derivative of the product in (1.14), using the Leibnitz rule, we get the following (bad) term

$$
\begin{aligned}
& \int\left(\pi_{N} u\right) \partial_{x}^{m-1}\left(\pi_{N} u\right) \pi_{>N}\left(\left(\pi_{N} u\right) \partial_{x}^{m}\left(\pi_{N} u\right)\right) d x \\
& =\int \pi_{>N}\left(\left(\pi_{N} u\right) \partial_{x}^{m-1}\left(\pi_{N} u\right)\right) \partial_{x}\left(\pi_{>N}\left(\left(\pi_{N} u\right) \partial_{x}^{m-1}\left(\pi_{N} u\right)\right)\right) d x \\
& \quad-\int \pi_{>N}\left(\left(\pi_{N} u\right) \partial_{x}^{m-1}\left(\pi_{N} u\right)\right) \pi_{>N}\left(\left(\pi_{N} \partial_{x} u\right) \partial_{x}^{m-1}\left(\pi_{N} u\right)\right) d x
\end{aligned}
$$

The worst term in the r.h.s. seems to be the first one, since it involves the product of a derivative of order $m$ and a derivative of order $m-1$. However this term is zero since it can be written as follows:

$$
\frac{1}{2} \int \partial_{x}\left(\pi_{>N}\left(\pi_{N} u\right) \partial_{x}^{m-1}\left(\pi_{N} u\right)\right)^{2} d x=0 .
$$

By looking at the structure of the conservation laws of the Benjamin-Ono equation, it is easy to check that the situation is a priori much worse. In fact if $E_{m}$ is the conservation law 
(for the Benjamin-Ono equation) with leading term $\|u\|_{H^{m}}^{2}$, and if we repeat the same construction as in (1.13) (where $p_{m}$ is replaced by the density of $E_{m}$ ), then the cubic part produces a contribution that involves a derivative of order $m$ which is very delicate since the Gaussian measure $d \mu_{m}$ is supported on $H^{m-1 / 2-\epsilon}$. Moreover, in $E_{m}$ the terms homogeneous of order four involve a derivative of order $m-1 / 2$ (this difficulty can be compared with the one we met above to treat the contribution coming from the cubic part of the conservation laws of $\mathrm{KdV}$ ). The second main result of this paper (Theorem 1.2) is essentially saying that we are able to find a key cancellation which eliminates the terms containing (at first glance) too many derivatives. We believe that this result is of independent interest and that it will play a role in the future analysis on the issues considered here.

Next we fix some notations.

Notation 1.3. - We shall denote by $H^{s}$ (and in some cases $H_{x}^{s}$ ) the Sobolev spaces of $2 \pi$-periodic functions;

$L^{p}$ (and in some cases $L_{x}^{p}$ ) is the $2 \pi$-periodic Lebesgue space;

$L_{\omega}^{q}$ is the Lebesgue space with respect to the probability measure $(\Omega, \mathscr{Q}, p)$, which in turn is the domain of definition of the random variables $\varphi_{n}(\omega)$ in (1.3);

if $f(x)$ is a $2 \pi$-periodic function then $\int f(x) d x=\int_{0}^{2 \pi} f(x) d x$;

the operator $H$ is the usual Hilbert transform acting on $2 \pi$-periodic functions;

for every $N \in \mathbb{N}$ the constant $\alpha_{N}$ is equal to $\sum_{n=1}^{N} \frac{1}{n}$;

for every $k \in N$ the quantities $E_{k / 2}$ and $R_{k / 2}$ are related as in (1.2), where $E_{k / 2}$ is a conservation law for the Benjamin-Ono equation.

Some other notations will be fixed in Section 2.

The remaining part of the paper is devoted to the proof of Theorems 1.1, 1.2.

\section{On the structure of the conservation laws of the Benjamin-Ono equation}

In this section, we describe the form of the Benjamin-Ono equation conservation laws which is suitable for the proof of our results announced in the introduction. Our reference in this discussion is the book by Matsuno [10].

We now fix some notations. Given any function $u(x) \in C^{\infty}\left(S^{1}\right)$, we set

$$
\begin{aligned}
& \mathscr{P}_{1}(u)=\left\{\partial_{x}^{\alpha_{1}} u, H \partial_{x}^{\alpha_{1}} u \mid \alpha_{1} \in \mathbb{N}\right\}, \\
& \mathscr{P}_{2}(u)=\left\{\partial_{x}^{\alpha_{1}} u \partial_{x}^{\alpha_{2}} u,\left(H \partial_{x}^{\alpha_{1}} u\right) \partial_{x}^{\alpha_{2}} u,\left(H \partial_{x}^{\alpha_{1}} u\right)\left(H \partial_{x}^{\alpha_{2}} u\right) \mid \alpha_{1}, \alpha_{2} \in \mathbb{N}\right\}
\end{aligned}
$$

and in general by induction

$$
\begin{aligned}
\mathscr{P}_{n}(u)=\left\{\prod_{l=1}^{k} H^{i_{l}} p_{j_{l}}(u) \mid i_{1}, \ldots, i_{k} \in\{0,1\},\right. \\
\left.\qquad \sum_{l=1}^{k} j_{l}=n, k \in\{2, \ldots, n\} \text { and } p_{j_{l}}(u) \in \mathscr{P}_{j_{l}}(u)\right\},
\end{aligned}
$$

where $H$ is again the Hilbert transform. 
ExAmple 2.1. - The elements belonging to $\mathscr{P}_{3}(u)$ are the following ones:

$$
\begin{gathered}
\partial_{x}^{\alpha_{1}} u \partial_{x}^{\alpha_{2}} u \partial_{x}^{\alpha_{3}} u, \partial_{x}^{\alpha_{1}} u \partial_{x}^{\alpha_{2}} u\left(H \partial_{x}^{\alpha_{3}} u\right), \partial_{x}^{\alpha_{1}} u H\left(\partial_{x}^{\alpha_{2}} u \partial_{x}^{\alpha_{3}} u\right), \\
\partial_{x}^{\alpha_{1}} u\left(H \partial_{x}^{\alpha_{2}} u\right)\left(H \partial_{x}^{\alpha_{3}} u\right), \partial_{x}^{\alpha_{1}} u H\left(\partial_{x}^{\alpha_{2}} u\left(H \partial_{x}^{\alpha_{3}} u\right)\right), H \partial_{x}^{\alpha_{1}} u H\left(\partial_{x}^{\alpha_{2}} u \partial_{x}^{\alpha_{3}} u\right), \\
\left(H \partial_{x}^{\alpha_{1}} u\right)\left(H \partial_{x}^{\alpha_{2}} u\right)\left(H \partial_{x}^{\alpha_{3}} u\right),\left(H \partial_{x}^{\alpha_{1}} u\right) H\left(\partial_{x}^{\alpha_{2}} u\left(H \partial_{x}^{\alpha_{3}} u\right)\right), \partial_{x}^{\alpha_{1}} u H\left(\left(H \partial_{x}^{\alpha_{2}} u\right)\left(H \partial_{x}^{\alpha_{3}} u\right)\right)
\end{gathered}
$$

where $\alpha_{1}, \alpha_{2}, \alpha_{3} \in \mathbb{N}$.

REMARK 2.2. - Roughly speaking an element in $\mathscr{P}_{n}(u)$ involves the product of $n$ derivatives $\partial_{x}^{\alpha_{1}} u, . ., \partial_{x}^{\alpha_{n}} u$ in combination with the Hilbert transform $H$ (that can appear essentially in an arbitrary way in front of the factors and eventually in front of a group of factors).

Notice that for every $n$ the simplest element belonging to $\mathscr{P}_{n}(u)$ has the following structure:

$$
\prod_{i=1}^{n} \partial_{x}^{\alpha_{i}} u, \alpha_{i} \in \mathbb{N} .
$$

In particular we can define the map

$$
\mathscr{P}_{n}(u) \ni p_{n}(u) \rightarrow \tilde{p}_{n}(u) \in \mathscr{P}_{n}(u)
$$

that associates to every $p_{n}(u) \in \mathscr{P}_{n}(u)$ the unique element $\tilde{p}_{n}(u) \in \mathscr{P}_{n}(u)$ having the structure given in (2.1) where $\partial_{x}^{\alpha_{1}} u, \partial_{x}^{\alpha_{2}} u, \ldots, \partial_{x}^{\alpha_{n}} u$ are the derivatives involved in the expression of $p_{n}(u)$ (equivalently $\tilde{p}_{n}(u)$ is obtained from $p_{n}(u)$ by erasing all the Hilbert transforms $H$ that appear in $\left.p_{n}(u)\right)$.

Next, we associate to every $p_{n}(u) \in \mathscr{P}_{n}(u)$ two integers as follows:

$$
\begin{aligned}
& \text { if } \tilde{p}_{n}(u)=\prod_{i=1}^{n} \partial_{x}^{\alpha_{i}} u \text { then } \\
&\left|p_{n}(u)\right|:=\sup _{i=1, . ., n} \alpha_{i}
\end{aligned}
$$

and

$$
\left\|p_{n}(u)\right\|:=\sum_{i=1}^{n} \alpha_{i} .
$$

We are ready to describe the structure of the conservation laws satisfied by the Benjamin-Ono equation. Given any even $k \in \mathbb{N}$, i.e., $k=2 n$, the energy $E_{k / 2}$ has the following structure:

$$
\begin{aligned}
E_{k / 2}(u)= & \|u\|_{\dot{H}^{k / 2}}^{2}+\sum_{\substack{p(u) \in \mathscr{P}_{3}(u) \text { s.t. } \\
\tilde{p}(u)=u \partial_{x}^{n-1} u \partial_{x}^{n} u}} c_{k}(p) \int p(u) d x \\
& +\sum_{\substack{p(u) \in \mathcal{P}_{j}(u) s . t . j=3, \ldots, 2 n+2 \\
\|p(u)\|=2 n-j+2 \\
|p(u)| \leq n-1}} c_{k}(p) \int p(u) d x \\
&
\end{aligned}
$$

where $c_{k}(p) \in \mathbb{R}$ are suitable real numbers. 
Similarly in the case of odd $k \in \mathbb{N}$, i.e., $k=2 n+1$, the energy $E_{k / 2}$ has the following structure:

$$
\begin{aligned}
E_{k / 2}(u)= & \|u\|_{\dot{H}^{k / 2}}^{2}+\sum_{\substack{p(u) \in \mathscr{P}_{3}(u) s . t . \\
\tilde{p}(u)=u \partial_{x}^{n} u \partial_{x}^{n} u}} c_{k}(p) \int p(u) d x \\
& +\sum_{\substack{p(u) \in \mathscr{P}_{3}(u) \text { s.t. } \\
\tilde{p}(u)=\partial_{x} u \partial_{x}^{n-1} u \partial_{x}^{n} u}} c_{k}(p) \int p(u) d x \\
& +\sum_{\substack{p(u) \in \mathscr{P}_{4}(u) \text { s.t. } \\
\tilde{p}(u)=u^{2} \partial_{x}^{n-1} u \partial_{x}^{n} u}} c_{k}(p) \int p(u) d x \\
& +\sum_{\substack{p(u) \in \mathscr{P}_{j}(u) s . t . j=3, \ldots, 2 n+3 \\
\|p(u)\|=2 n-j+3 \\
|p(u)| \leq n-1}} c_{k}(p) \int p(u) d x
\end{aligned}
$$

where $c_{k}(p) \in \mathbb{R}$ are suitable real numbers.

REMARK 2.3. - The expressions above should be compared with the explicit structure of $E_{k / 2}$ for $k=0,1,2,3,4$ (see the introduction).

\section{Preliminary estimates}

Along this section we shall use the notations $\mathscr{P}_{j}(u), p_{j}(u), \tilde{p}_{j}(u)$ introduced in Section 2. We also recall that $E_{k / 2}$ denotes the conservation law whose structure is described in (2.4) and (2.5) (respectively depending on the evenness or oddness of $k$ ). The main result of this section is the following proposition that will be very useful to prove Theorem 1.1 for $k$ an even number.

Proposition 3.1. - Let $k>0$ be a given integer. Then for every $R_{1}, R_{2}>0$ there is $C=C\left(R_{1}, R_{2}\right)>0$ such that

$$
\begin{aligned}
& \bigcap_{j=0}^{2 k}\left\{u \in H^{k}|| E_{j / 2}(u) \mid<R_{1}\right\} \cap\left\{u \in H^{k}|| E_{k+1 / 2}\left(\pi_{N} u\right)-\alpha_{N} \mid<R_{2}\right\} \\
& \quad \subset\left\{u \in H^{k} \mid\|u\|_{H^{k}}<C\right\} \cap\left\{u \in H^{k}\left|\left\|\pi_{N} u\right\|_{\dot{H}^{k+1 / 2}}^{2}-\alpha_{N}\right|<C\right\}, \forall N \in \mathbb{N} .
\end{aligned}
$$

REMARK 3.2. - The proposition above (where we choose $k=m$ ) implies that the support of the functions $F_{m+1, N, R}$ defined in (1.5) is contained in a ball of $H^{m}$ intersected with the region $\left\{u \in H^{m}\left|\left\|\pi_{N} u\right\|_{\dot{H}^{m+1 / 2}}^{2}-\alpha_{N}\right|<C\right\}$ (at least in the case $m>0$ ).

Lemma 3.3. - For every integer $m \geq 0$ there exists $C=C(m)>0$ such that

$$
\begin{aligned}
& \left|\int u \partial_{x}^{m} v \partial_{x}^{m+1} w d x\right| \leq C\left(\|u\|_{L^{\infty}}\|v\|_{H^{m+1 / 2}}\|w\|_{H^{m+1 / 2}}\right. \\
& \left.+\|v\|_{L^{\infty}}\|u\|_{H^{m+1 / 2}}\|w\|_{H^{m+1 / 2}}+\|w\|_{L^{\infty}}\|u\|_{H^{m+1 / 2}}\|v\|_{H^{m+1 / 2}}\right) .
\end{aligned}
$$


Proof. - We consider a Littlewood-Paley partition of unity $1=\sum_{N} \Delta_{N}$, where $N$ takes the dyadic values, i.e., $N=2^{j}, j=0,1,2, \cdots$. We denote by $S_{N}$ the operator $\sum_{N_{1} \leq N} \Delta_{N_{1}}$. In order to prove (3.2), one needs to evaluate the expression

$$
\sum_{N_{1}, N_{2}, N_{3}} \int \Delta_{N_{1}} u \partial_{x}^{m} \Delta_{N_{2}} v \partial_{x}^{m+1} \Delta_{N_{3}} w d x
$$

We consider three cases by distinguishing which is the smallest of $N_{1}, N_{2}$ and $N_{3}$. In the sequel we shall denote by $c, C>0$ constants that can change at each step.

Denote by $J_{1}$, the contribution of $N_{1} \leq \min \left(N_{2}, N_{3}\right)$ to (3.3). Then $N_{2} \sim N_{3}$ and

$$
\begin{aligned}
J_{1} & \leq C \sum_{N_{2} \sim N_{3}}\left|\int S_{c \min \left(N_{2}, N_{3}\right)} u \partial_{x}^{m} \Delta_{N_{2}} v \partial_{x}^{m+1} \Delta_{N_{3}} w d x\right| \\
& \leq C \sum_{N_{2} \sim N_{3}}\|u\|_{L^{\infty}} N_{2}^{m}\left\|\Delta_{N_{2}} v\right\|_{L^{2}} N_{3}^{m+1}\left\|\Delta_{N_{3}} w\right\|_{L^{2}} \\
& \leq C\|u\|_{L^{\infty}}\|v\|_{H^{m+1 / 2}}\|w\|_{H^{m+1 / 2}},
\end{aligned}
$$

where in the last line we used the Cauchy-Schwarz inequality. Next denote by $J_{2}$ the contribution of $N_{2} \leq \min \left(N_{1}, N_{3}\right)$ to (3.3). Then

$$
\begin{aligned}
J_{2} & \leq C \sum_{N_{1} \sim N_{3}}\left|\int \Delta_{N_{1}} u \partial_{x}^{m} S_{c \min \left(N_{1}, N_{3}\right)} v \partial_{x}^{m+1} \Delta_{N_{3}} w d x\right| \\
& \leq C \sum_{N_{1} \sim N_{3}}\left\|\Delta_{N_{1}} u\right\|_{L^{2}}\left(\min \left(N_{1}, N_{3}\right)\right)^{m}\|v\|_{L^{\infty}} N_{3}^{m+1}\left\|\Delta_{N_{3}} w\right\|_{L^{2}} \\
& \leq C\|v\|_{L^{\infty}}\|u\|_{H^{m+1 / 2}}\|w\|_{H^{m+1 / 2}} .
\end{aligned}
$$

Finally, we denote by $J_{3}$, the contribution of $N_{3} \leq \min \left(N_{1}, N_{2}\right)$ to (3.3). Then

$$
\begin{aligned}
J_{3} & \leq C \sum_{N_{1} \sim N_{2}}\left|\int \Delta_{N_{1}} u \partial_{x}^{m} \Delta_{N_{2}} v \partial_{x}^{m+1} S_{c \min \left(N_{1}, N_{2}\right)} w d x\right| \\
& \leq C \sum_{N_{1} \sim N_{2}}\left\|\Delta_{N_{1}} u\right\|_{L^{2}} N_{2}^{m}\left\|\Delta_{N_{2}} v\right\|_{L^{2}}\left(\min \left(N_{1}, N_{2}\right)\right)^{m+1}\|w\|_{L^{\infty}} \\
& \leq C\|w\|_{L^{\infty}}\|u\|_{H^{m+1 / 2}}\|v\|_{H^{m+1 / 2}} .
\end{aligned}
$$

This completes the proof of Lemma 3.3.

As a consequence of Lemma 3.3 we get the following useful result.

Lemma 3.4. - Let $m \geq 0$ be an integer and $p_{3}(u) \in \mathscr{P}_{3}(u)$ be such that

$$
\tilde{p}_{3}(u)=u \partial_{x}^{m} u \partial_{x}^{m+1} u
$$

Then for every $\epsilon>0,1<p<\infty$ such that $\epsilon p>1$, there exists $C=C(\epsilon, p)>0$ such that:

$$
\left|\int p_{3}(u) d x\right| \leq C\|u\|_{H^{m+1 / 2}}^{2}\|u\|_{W^{\epsilon, p}} .
$$

Proof. - Looking at the structure of the elements in $\mathscr{D}_{3}(u)$ and since we are assuming (3.4) we can deduce by Lemma 3.3 the following estimate:

$$
\left|\int p_{3}(u) d x\right| \leq C\left(\max \left\{\|u\|_{H^{m+1 / 2}},\|H u\|_{H^{m+1 / 2}}\right\}\right)^{2} \max \left\{\|u\|_{L^{\infty}},\|H u\|_{L^{\infty}}\right\}
$$


and hence by the Sobolev embedding $W^{\epsilon, p} \subset L^{\infty}$ we can continue the estimate as follows (provided that we change the constant $C$ )

$$
\cdots \leq C\|u\|_{H^{m+1 / 2}}^{2} \max \left\{\|u\|_{W^{\epsilon, p}},\|H u\|_{W^{\epsilon, p}}\right\} .
$$

The proof can be completed since the Hilbert transform $H$ is continuous in the spaces $L^{p}$ for $1<p<\infty$.

Lemma 3.5. - Let $k \geq 2$ be an integer. For every $p_{3}(u) \in \mathscr{P}_{3}(u)$ such that

$$
\tilde{p}_{3}(u)=\prod_{i=1}^{3} \partial_{x}^{\alpha_{i}} u \text { with } 0 \leq \sum_{i=1}^{3} \alpha_{i}=2 k+1 \text { and } 1 \leq \min _{i=1,2,3} \alpha_{i} \leq \max _{i=1,2,3} \alpha_{i} \leq k
$$

we have:

$$
\left|\int p_{3}(u) d x\right| \leq C\|u\|_{H^{k}}^{3}
$$

Proof. - We can assume $\alpha_{1} \geq \alpha_{2} \geq \alpha_{3}$ and also $p_{3}(u)=\prod_{i=1}^{3} \partial_{x}^{\alpha_{i}} u$ (the general case follows in a similar way).

First case: $\alpha_{1}=\alpha_{2}=k$.

In this case necessarily $\alpha_{3}=1$ and hence by the Hölder inequality we get

$$
\left|\int p_{3}(u) d x\right| \leq\|u\|_{H^{k}}^{2}\left\|\partial_{x} u\right\|_{L^{\infty}} \leq C\|u\|_{H^{k}}^{3}
$$

where we have used the Sobolev embedding $H^{1} \subset L^{\infty}$.

Second case: $\alpha_{2} \leq k-1$.

By the Hölder inequality we get

$$
\left|\int p_{3}(u) d x\right| \leq\|u\|_{H^{k}}\|u\|_{H^{k-1}}\left\|\partial_{x}^{\alpha_{3}}\right\|_{L^{\infty}}
$$

and hence by the embedding $H^{1} \subset L^{\infty}$

$$
\cdots \leq C\|u\|_{H^{k}}^{2}\|u\|_{H^{\alpha_{3}+1}} .
$$

The proof follows since $\alpha_{3}+1 \leq \alpha_{2}+1 \leq k$.

Lemma 3.6. - Let $k \geq 1$ and $j \geq 3$ be integers. For every $p_{j}(u) \in \mathscr{P}_{j}(u)$ such that

$$
\tilde{p}_{j}(u)=\prod_{i=1}^{j} \partial_{x}^{\alpha_{i}} u \text { with } 0 \leq \sum_{i=1}^{j} \alpha_{i} \leq 2 k
$$

we have:

$$
\left|\int p_{j}(u) d x\right| \leq C\|u\|_{H^{k}}^{j}
$$


Proof. - We treat explicitly the case $p_{j}(u)=\tilde{p}_{j}(u)$ (we specify shortly below how to treat the general case).

It is not restrictive to assume that

$$
\alpha_{1} \geq \alpha_{2} \geq \cdots \geq \alpha_{j}
$$

By eventually performing integrations by parts we can assume $\alpha_{1} \leq k$ and by (3.7) also $\alpha_{2} \leq k$. Moreover by the assumption we get

$$
\alpha_{i}<k, \forall i=3, \ldots, j .
$$

Hence by the Hölder inequality we get:

$$
\left|\int p_{j}(u) d x\right| \leq\left\|\partial_{x}^{\alpha_{1}} u\right\|_{L^{2}}\left\|\partial_{x}^{\alpha_{2}} u\right\|_{L^{2}} \prod_{i=3}^{j}\left\|\partial_{x}^{\alpha_{i}} u\right\|_{L^{\infty}}
$$

which due to the embedding $H^{1} \subset L^{\infty}$ and (3.8) implies (3.6) (if the Hilbert transform $H$ is involved in the expression of $p_{j}(u)$ then we are allowed to remove $H$ at the last step since $\left.\|H u\|_{H^{s}}=\|u\|_{H^{s}}\right)$.

Lemma 3.7. - Let $n \geq 0$ be an integer and $R>0$, then

$$
\exists C=C(n, R)>0 \text { s.t. } \bigcap_{j=0}^{2 n}\left\{u \in H^{n}|| E_{j / 2}(u) \mid<R\right\} \subset\left\{u \in H^{n} \mid\|u\|_{H^{n}}<C\right\} .
$$

Proof. - We use induction on $n$.

First step: $n=0$.

This is trivial since $E_{0}(u)=\|u\|_{L^{2}}^{2}$.

Second step: $n=1$.

By combining the explicit structure of $E_{1 / 2}$ (see the introduction) with the following inequality

we get

$$
\|u\|_{L^{3}} \leq\|u\|_{L^{2}}^{1 / 2}\|u\|_{L^{6}}^{1 / 2} \leq C\|u\|_{L^{2}}^{1 / 2}\|u\|_{H^{1 / 2}}^{1 / 2}
$$

(see the notation in (1.2)). Hence in the region

$$
\left\{u \in H^{1}|| E_{1 / 2}(u)|<R,| E_{0}(u) \mid<R\right\}
$$

we get

$$
\|u\|_{\dot{H}^{1 / 2}}^{2}=\left|E_{1 / 2}(u)-R_{1 / 2}(u)\right| \leq R+R^{3 / 4}\|u\|_{H^{1 / 2}}^{3 / 2}
$$

which in turn implies the existence of $C>0$ such that

$$
\|u\|_{H^{1 / 2}}<C, \forall u \in\left\{u \in H^{1}|| E_{1 / 2}(u)|<R,| E_{0}(u) \mid<R\right\} .
$$

Next, by looking at the explicit structure of $E_{1}$ (see the introduction) we get

$$
\left|R_{1}(u)\right| \leq C\|u\|_{H^{1}}\|u\|_{H^{1 / 2}}^{2}+C\|u\|_{H^{1 / 2}}^{4}
$$

(see the notation (1.2)) where we have used the Sobolev embedding $H^{1 / 2} \subset L^{4}$. Hence by (3.12) we get a suitable constant $C>0$ such that

$$
\|u\|_{\dot{H}^{1}}^{2}=\left|E_{1}(u)-R_{1}(u)\right| \leq R+C+C\|u\|_{H^{1}}
$$




$$
\forall u \in\left\{u \in H^{1}|| E_{1}(u)|<R,| E_{1 / 2}(u)|<R,| E_{0}(u) \mid<R\right\} .
$$

In turn, this implies the existence of $C>0$ such that

$$
\|u\|_{H^{1}}<C, \forall u \in\left\{u \in H^{1}|| E_{1}(u)|<R,| E_{1 / 2}(u)|<R,| E_{0}(u) \mid<R\right\} .
$$

Third step: $n=2$.

Following the argument of the previous step we get

$$
\|u\|_{H^{1}}<C, \forall u \in\left\{u \in H^{2}|| E_{1}(u)|<R,| E_{1 / 2}(u)|<R,| E_{0}(u) \mid<R\right\}
$$

for a suitable $C>0$. By combining the structure of $E_{3 / 2}$ (see the introduction) with (3.13) and the Sobolev embedding $H^{1} \subset L^{\infty}$ we get

$$
\left|R_{3 / 2}(u)\right|<C, \forall u \in\left\{u \in H^{2}|| E_{1}(u)|<R,| E_{1 / 2}(u)|<R,| E_{0}(u) \mid<R\right\} .
$$

As a consequence we deduce

$$
\begin{gathered}
\|u\|_{\dot{H}^{3 / 2}}^{2}=\left|E_{3 / 2}(u)-R_{3 / 2}(u)\right| \leq R+C \\
\forall u \in\left\{u \in H^{2} \| E_{3 / 2}(u)|<R,| E_{1}(u)|<R,| E_{1 / 2}(u)|<R,| E_{0}(u) \mid<R\right\} .
\end{gathered}
$$

By combining (3.14) with Lemma 3.4 and Lemma 3.6 we get

$$
\begin{gathered}
\left|R_{2}(u)+\frac{5}{4} \int\left(u_{x}\right)^{2} H u_{x} d x\right|<C \\
\forall u \in\left\{u \in H^{2}|| E_{3 / 2}(u)|<R,| E_{1}(u)|<R,| E_{1 / 2}(u)|<R,| E_{0}(u) \mid<R\right\}
\end{gathered}
$$

and hence

$$
\begin{aligned}
\|u\|_{H^{2}}^{2} & =E_{2}(u)+\left(R_{2}(u)+\frac{5}{4} \int\left(u_{x}\right)^{2} H u_{x} d x\right)-\frac{5}{4} \int\left(u_{x}\right)^{2} H u_{x} d x \\
& \leq R+C+\|u\|_{H^{1}}^{2}\|u\|_{H^{2}} \leq R+C+C\|u\|_{H^{2}}
\end{aligned}
$$

for all $u$ in $\bigcap_{j=0}^{4}\left\{u \in H^{2}|| E_{j / 2}(u) \mid<R\right\}$, where we have used the Hölder inequality and the Sobolev embedding $H^{1} \subset L^{\infty}$ to estimate the integral $\int\left(u_{x}\right)^{2} H u_{x} d x$. The proof can be easily concluded.

Fourth step: $n \Rightarrow n+1$ for $n \geq 2$.

Assume the conclusion is proved for $n \geq 1$, then there exists $C>0$ such that

$$
\begin{aligned}
\bigcap_{j=0}^{2(n+1)}\left\{u \in H^{n+1} \| E_{j / 2}(u) \mid<R\right\} & \subset \bigcap_{j=0}^{2 n}\left\{u \in H^{n+1}|| E_{j / 2}(u) \mid<R\right\} \\
& \subset\left\{u \in H^{n+1} \mid\|u\|_{H^{n}}<C\right\} .
\end{aligned}
$$

Next we shall use (following (1.2)) the notation

$$
E_{n+1 / 2}(u)=\|u\|_{\dot{H}^{n+1 / 2}}^{2}+R_{n+1 / 2}(u)
$$

and

$$
E_{n+1}(u)=\|u\|_{\dot{H}^{n+1}}^{2}+R_{n+1}(u)
$$

(the structure of $R_{k / 2}$, described in (2.4) and (2.5), depending on the evenness or the oddness of $k$, will be freely exploited in the sequel). 
By combining Lemma 3.6 (where we choose $k=n$ ), with (3.15) we deduce

$$
\left|R_{n+1 / 2}(u)\right|<C, \forall u \in \bigcap_{j=0}^{2(n+1)}\left\{u \in H^{n+1}|| E_{j / 2}(u) \mid<R\right\}
$$

for a suitable $C>0$, where we have used the fact that $R_{n+1 / 2}(u)$ involves terms of the type $\int p_{j}(u) d x$ with $j \geq 3$ and $\left\|p_{j}(u)\right\| \leq 2 n$ (for a definition of $\left\|p_{j}(u)\right\|$ see (2.3)). As a consequence of (3.16) and (3.18) we get

$$
\bigcap_{j=0}^{2(n+1)}\left\{u \in H^{n+1}|| E_{j / 2}(u) \mid<R\right\} \subset\left\{u \in H^{n+1} \mid\|u\|_{H^{n+1 / 2}}<C\right\} .
$$

By combining Lemma 3.4 (where we choose $m=n, \epsilon=1, p=2$ ), Lemma 3.5, Lemma 3.6 (with $k=n$ ) and (3.19) we deduce

$$
\left|R_{n+1}(u)\right|<C+C\|u\|_{H^{n+1}}, \forall u \in \bigcap_{j=0}^{2(n+1)}\left\{u \in H^{n+1}|| E_{j / 2}(u) \mid<R\right\}
$$

(where we have used the structure of $R_{n+1}$ given in (2.4)). By combining this estimate with (3.17) we get

$$
\begin{gathered}
\|u\|_{H^{n+1}}^{2} \leq\left|E_{n+1}(u)\right|+C+C\|u\|_{H^{n+1}} \leq R+C+C\|u\|_{H^{n+1}} \\
\forall u \in \bigcap_{j=0}^{2(n+1)}\left\{u \in H^{n+1}|| E_{j / 2}(u) \mid<R\right\}
\end{gathered}
$$

which in turn implies (3.10) for $n+1$.

Proof of Proposition 3.1. - By (3.10) (where we choose $n=k$ ) there exists $C>0$ such that

$$
\|u\|_{H^{k}}<C, \forall u \in \bigcap_{j=0}^{2 k}\left\{u \in H^{k}|| E_{j / 2}(u) \mid<R_{1}\right\} .
$$

We also recall the notation (see (1.2))

$$
E_{k+1 / 2}(u)=\|u\|_{\dot{H}^{k+1 / 2}}^{2}+R_{k+1 / 2}(u) .
$$

By combining (2.5) with (3.20) and Lemma 3.6 (recall that we are assuming $k>0$ ) we get that for every $R$ there exists $C=C(R)$ such that

$$
\left|R_{k+1 / 2}(u)\right|<C, \forall u \in\left\{u \in H^{k} \mid\|u\|_{H^{k}}<R\right\}
$$

which is equivalent to

$$
\left|E_{k+1 / 2}(u)-\|u\|_{\dot{H}^{k+1 / 2}}^{2}\right|<C, \forall u \in\left\{u \in H^{k} \mid\|u\|_{H^{k}}<R\right\}
$$

and hence

$$
\begin{aligned}
& \left|E_{k+1 / 2}\left(\pi_{N} u\right)-\left\|\pi_{N} u\right\|_{\dot{H}^{k+1 / 2}}^{2}\right|<C \\
& \forall u \in\left\{u \in H^{k} \mid\|u\|_{H^{k}}<R\right\}, N \in \mathbb{N} .
\end{aligned}
$$

By (3.20) we get

$$
\left|E_{k+1 / 2}\left(\pi_{N} u\right)-\left\|\pi_{N} u\right\|_{\dot{H}^{k+1 / 2}}^{2}\right|<C
$$

$4^{\text {e }}$ SÉRIE - TOME $46-2013$ - No 2 


$$
\forall u \in \bigcap_{j=0}^{2 k}\left\{u \in H^{k}|| E_{j / 2}(u) \mid<R_{1}\right\}, N \in \mathbb{N}
$$

that in turn implies (3.1).

\section{A linear Gaussian bound}

We start with the following general measure theory result which shall be frequently used in the sequel.

Proposition 4.1. - Let $F:(\Omega, \mathscr{G}, p) \rightarrow \mathbf{C}$ be measurable and $C, \alpha>0$ be such that

$$
\|F\|_{L^{q}} \leq C q^{\alpha}, \forall q \in[1, \infty) .
$$

Then

$$
p\{\omega \in \Omega|| F(\omega) \mid>\lambda\} \leq e^{-\frac{\alpha}{e}\left(\frac{\lambda}{C}\right)^{\frac{1}{\alpha}}}, \forall \lambda>0
$$

Proof. - By combining the Tchebychev inequality with (4.1) we get:

$$
p\{\omega \in \Omega|| F(\omega) \mid>\lambda\} \leq \frac{\|F\|_{q}^{q}}{\lambda^{q}} \leq C^{q}\left(\frac{q^{\alpha}}{\lambda}\right)^{q} .
$$

We conclude by choosing $q=\left(\frac{\lambda}{C}\right)^{\frac{1}{\alpha}} e^{-1}$.

Next we present, as an application of the previous result, a linear Gaussian bound which will be used in the next sections.

Proposition 4.2. - For every integer $m \geq 0,0<\epsilon<\frac{1}{2}$ and $1 \leq p<\infty$ there exists $C=C(m, \epsilon, p)>0$ such that

$$
p\left\{\omega \in \Omega \mid\left\|\varphi_{m+1}(\omega)\right\|_{W_{x}^{\epsilon, p}}>\lambda\right\} \leq C e^{-\frac{\lambda^{2}}{C}}, \forall \lambda>0
$$

where $\varphi_{m+1}(\omega)$ is the random vector in (1.3) for $k=2(m+1)$.

Proof. - It is sufficient to prove that

$$
p\left\{\omega \in \Omega \mid\left\|\sum_{k>0} \frac{\varphi_{k}(\omega)}{k^{m+1-\epsilon}} e^{i k x}\right\|_{L_{x}^{p}}>\lambda\right\} \leq C e^{-\frac{\lambda^{2}}{C}} .
$$

For every fixed $x \in(0,2 \pi)$ the random variable

$$
\sum_{k>0} \frac{\varphi_{k}(\omega)}{k^{m+1-\epsilon}} e^{i k x}
$$

is Gaussian and its distribution function is $\frac{1}{\pi K} e^{-\frac{|z|^{2}}{K}} d z$ where $K=\sum_{k>0} \frac{1}{k^{2(m+1-\epsilon)}}$.

As a consequence we get the following estimate:

$$
\begin{aligned}
\left\|\sum_{k>0} \frac{\varphi_{k}(\omega)}{k^{m+1-\epsilon}} e^{i k x}\right\|_{L_{\omega}^{q}}^{q} & =\frac{1}{\pi K} \int_{\mathbb{C}}|z|^{q} e^{-\frac{|z|^{2}}{K}} d z \\
& =2 K^{\frac{q}{2}} \int_{0}^{\infty} e^{-s^{2}} s^{q+1} d s \leq C K^{\frac{q}{2}}\left(\frac{q}{2}\right)^{\frac{q}{2}}, \forall x \in(0,2 \pi)
\end{aligned}
$$


for a suitable $C>0$ (the last inequality can be proved by integration by parts). In particular we get:

$$
\left\|\sum_{k>0} \frac{\varphi_{k}(\omega)}{k^{m+1-\epsilon}} e^{i k x}\right\|_{L_{\omega}^{q}} \leq C \sqrt{q}, \forall x \in(0,2 \pi)
$$

and hence

$$
\left\|\sum_{k>0} \frac{\varphi_{k}(\omega)}{k^{m+1-\epsilon}} e^{i k x}\right\|_{L_{x}^{p} L_{\omega}^{q}} \leq C \sqrt{q} .
$$

Due to the inequality $\|\cdot\|_{L_{\omega}^{q} L_{x}^{p}} \leq\|\cdot\|_{L_{x}^{p} L_{\omega}^{q}}$ for every $q \geq p$ we get:

$$
\left\|\sum_{k>0} \frac{\varphi_{k}(\omega)}{k^{m+1-\epsilon}} e^{i k x}\right\|_{L_{\omega}^{q} L_{x}^{p}} \leq C \sqrt{q}, \forall q \geq p
$$

(since $p(\Omega)=1$ it is easy to deduce that the estimate above is true for every $q \geq 1$ eventually with a new constant $C$ ). Hence we can conclude by using Proposition 4.1.

\section{Multilinear Gaussian bounds}

For any $p(u) \in \cup_{j=1}^{\infty} \mathscr{P}_{j}(u)$ (see Section 2) and for any $N \in \mathbb{N}$ we introduce the functions

$$
f^{p}(v)=\int p(v) d x \text { and } f_{N}^{p}(v)=\int p\left(\pi_{N} v\right) d x .
$$

We also recall that the Sobolev spaces $H^{m+1 / 2-\epsilon}$ are a support for the Gaussian measure $d \mu_{m+1}$ for every $\epsilon>0$. This fact will be used without any further comment in the sequel. The main results of this section are the following propositions.

Proposition 5.1. - Let $m \geq 0$ and $p_{3}(u) \in \mathscr{P}_{3}(u)$ be such that $\tilde{p}_{3}(u)=u \partial_{x}^{m} u \partial_{x}^{m+1} u$. Then there exists $C>0$ such that

$$
\left\|f_{N}^{p_{3}}(u)-f_{M}^{p_{3}}(u)\right\|_{L^{p}\left(d \mu_{m+1}\right)} \leq C \frac{p^{3 / 2}}{\sqrt{\min \{M, N\}}}, \forall M, N \in \mathbb{N}, p \geq 2 .
$$

In particular

$$
\exists C>0 \text { s.t. } \mu_{m+1}\left(A_{M, N}^{p_{3}, \lambda}\right) \leq e^{-\frac{1}{C}(\lambda \sqrt{\min \{N, M\}})^{2 / 3}}, \forall M, N \in \mathbb{N}, \lambda>0
$$

where

$$
A_{M, N}^{p_{3}, \lambda}=\left\{u \in H^{m+1 / 2-\epsilon}|| f_{N}^{p_{3}}(u)-f_{M}^{p_{3}}(u) \mid>\lambda\right\} .
$$

Proposition 5.2. - Let $m \geq 0$ be fixed. There exists $C>0$ such that

$$
\left\|h_{N}(u)-h_{M}(u)\right\|_{L^{p}\left(d \mu_{m+1}\right)} \leq C \frac{p}{\sqrt{\min \{M, N\}}}, \forall M, N \in \mathbb{N}, p \geq 2
$$

where $h_{K}(u)=\left\|\pi_{K} u\right\|_{\dot{H}^{m+1 / 2}}^{2}-\alpha_{K}$ for any $K \in \mathbb{N}$. In particular

$$
\exists C>0 \text { s.t. } \mu_{m+1}\left(B_{M, N}^{\lambda}\right) \leq e^{-\frac{1}{C}(\lambda \sqrt{\min \{N, M\}})}, \forall M, N \in \mathbb{N}, \lambda>0
$$

where

$$
B_{M, N}^{\lambda}=\left\{u \in H^{m+1 / 2-\epsilon}|| h_{N}(u)-h_{M}(u) \mid>\lambda\right\} .
$$


We need some preliminary lemmas. The first one concerns the orthogonality of the functions $\left\{\varphi_{i}(\omega) \varphi_{j}(\omega) \varphi_{k}(\omega)\right\}_{i, j, k \in \mathbb{Z}}$ (where $\varphi_{n}(\omega)$ are the Gaussian functions that appear in (1.3)) provided that $(i, j, k) \in \mathscr{G}$, where

$$
\mathscr{G}=\{(i, j, k) \in \mathbb{Z} \backslash\{0\} \mid i+j+k=0\} .
$$

Lemma 5.3. - Let $\left(k_{1}, k_{2}, k_{3}\right),\left(j_{1}, j_{2}, j_{3}\right) \in \mathscr{C}$ be such that

$$
\left\{k_{1}, k_{2}, k_{3}\right\} \neq\left\{j_{1}, j_{2}, j_{3}\right\} .
$$

Then

$$
\int_{\Omega} \varphi_{k_{1}} \varphi_{k_{2}} \varphi_{k_{3}} \overline{\varphi_{j_{1}} \varphi_{j_{2}} \varphi_{j_{3}}} d \omega=0
$$

Proof. - We split the proof in two cases (which in turn are splited in several subcases).

First case: $\exists i \in\{1,2,3\}$ s.t. $k_{i} \notin\left\{j_{1}, j_{2}, j_{3}\right\}$.

We can assume

$$
k_{1} \notin\left\{j_{1}, j_{2}, j_{3}\right\}
$$

Next we consider four subcases:

First subcase:

$$
k_{1} \notin\left\{k_{2}, k_{3}\right\} \text { and }-k_{1} \notin\left\{j_{1}, j_{2}, j_{3}\right\} .
$$

Notice that by definition of $\mathscr{G}$ necessarily

$$
-k_{1} \notin\left\{k_{2}, k_{3}\right\} \text {. }
$$

Hence by combining (5.9), (5.10), (5.11) and the independence assumption on $\left\{\varphi_{n}(\omega)\right\}_{n>0}$ we get:

$$
0=\int \varphi_{k_{1}} \int \varphi_{k_{2}} \varphi_{k_{3}} \overline{\varphi_{j_{1}} \varphi_{j_{2}} \varphi_{j_{3}}} d \omega=\int \varphi_{k_{1}} \varphi_{k_{2}} \varphi_{k_{3}} \overline{\varphi_{j_{1}} \varphi_{j_{2}} \varphi_{j_{3}}} d \omega
$$

Second subcase:

$$
k_{1} \in\left\{k_{2}, k_{3}\right\} \text { and }-k_{1} \notin\left\{j_{1}, j_{2}, j_{3}\right\} .
$$

It is not restrictive to assume

$$
k_{1}=k_{2}
$$

and hence by the definition of $\mathscr{G}$

$$
k_{3} \neq \pm k_{1} \text {. }
$$

Hence by combining (5.9), (5.12), (5.13), (5.14) with the independence assumption on $\left\{\varphi_{n}(\omega)\right\}_{n>0}$ we get

$$
0=\int \varphi_{k_{1}}^{2} d \omega \int \varphi_{k_{3}} \overline{\varphi_{j_{1}} \varphi_{j_{2}} \varphi_{j_{3}}} d \omega=\int \varphi_{k_{1}} \varphi_{k_{2}} \varphi_{k_{3}} \overline{\varphi_{j_{1}} \varphi_{j_{2}} \varphi_{j_{3}}} d \omega
$$

Third subcase:

$$
k_{1} \notin\left\{k_{2}, k_{3}\right\} \text { and }-k_{1} \in\left\{j_{1}, j_{2}, j_{3}\right\} .
$$

By definition of $\mathscr{G}$ we also deduce

$$
-k_{1} \notin\left\{k_{2}, k_{3}\right\}
$$


By (5.15) we can assume for simplicity either

$$
-k_{1}=j_{1} \notin\left\{j_{2}, j_{3}\right\}
$$

or

$$
-k_{1}=j_{1}=j_{2} \text {. }
$$

In the case when (5.17) occurs, we can also assume by the definition of $\mathscr{C}$ that

$$
k_{1}=-j_{1} \notin\left\{j_{2}, j_{3}\right\} \text {. }
$$

By combining (5.9), (5.15), (5.16), (5.17), (5.19) with the independence assumption on $\left\{\varphi_{n}(\omega)\right\}_{n>0}$ we get

$$
0=\int \varphi_{k_{1}}^{2} d \omega \int \varphi_{k_{2}} \varphi_{k_{3}} \overline{\varphi_{j_{2}} \varphi_{j_{3}}} d \omega=\int \varphi_{k_{1}} \varphi_{k_{2}} \varphi_{k_{3}} \overline{\varphi_{j_{1}} \varphi_{j_{2}} \varphi_{j_{3}}} d \omega
$$

(where we have also used (5.9)); in the case when (5.18) occurs, by using the definition of $\mathscr{C}$ we get

$$
\pm k_{1} \neq j_{3}
$$

Hence by combining (5.15), (5.16), (5.18), (5.20) we deduce

$$
0=\int \varphi_{k_{1}}^{3} d \omega \int \varphi_{k_{2}} \varphi_{k_{3}} \overline{\varphi_{j_{3}}} d \omega=\int \varphi_{k_{1}} \varphi_{k_{2}} \varphi_{k_{3}} \overline{\varphi_{j_{1}} \varphi_{j_{2}} \varphi_{j_{3}}} d \omega
$$

Fourth subcase:

$$
k_{1} \in\left\{k_{2}, k_{3}\right\} \text { and }-k_{1} \in\left\{j_{1}, j_{2}, j_{3}\right\} .
$$

We can assume

$$
k_{1}=k_{2}
$$

and by the definition of $\mathscr{C}$ also

$$
k_{3} \neq \pm k_{1}
$$

Moreover, we can assume that either

$$
-k_{1}=j_{1} \notin\left\{j_{2}, j_{3}\right\}
$$

or

$$
-k_{1}=j_{1}=j_{2} \text {. }
$$

In the case when (5.24) occurs we can also assume by the definition of $\mathscr{G}$ that

$$
k_{1}=-j_{1} \notin\left\{j_{2}, j_{3}\right\} .
$$

Hence by combining (5.22), (5.23), (5.24), (5.26) we get

$$
0=\int \varphi_{k_{1}}^{3} d \omega \int \varphi_{k_{3}} \overline{\varphi_{j_{2}} \varphi_{j_{3}}} d \omega=\int \varphi_{k_{1}} \varphi_{k_{2}} \varphi_{k_{3}} \overline{\varphi_{j_{1}} \varphi_{j_{2}} \varphi_{j_{3}}} d \omega
$$

in the case when (5.25) occurs we can deduce by the definition of $\mathscr{Q}$ that

$$
\pm k_{1} \neq j_{3} \text {. }
$$

4 ${ }^{\mathrm{e}}$ SÉRIE - TOME 46 - 2013 - No 2 
Hence by (5.22), (5.23), (5.25), (5.27) we get

$$
0=\int \varphi_{k_{1}}^{4} d \omega \int \varphi_{k_{3}} \overline{\varphi_{j_{3}}} d \omega=\int \varphi_{k_{1}} \varphi_{k_{2}} \varphi_{k_{3}} \overline{\varphi_{j_{1}} \varphi_{j_{2}} \varphi_{j_{3}}} d \omega
$$

Second case:

$$
k_{i} \in\left\{j_{1}, j_{2}, j_{3}\right\} \forall i=1,2,3 .
$$

Next we consider two subcases:

First subcase:

$$
k_{1} \neq k_{2}, k_{1} \neq k_{3}, k_{2} \neq k_{3} .
$$

By combining (5.28) and (5.29) it is easy to deduce that

$$
\left\{j_{1}, j_{2}, j_{3}\right\}=\left\{k_{1}, k_{2}, k_{3}\right\}
$$

which is in contradiction with (5.8).

Second subcase:

$$
\exists n, m \in\{1,2,3\} \text { s.t. } n \neq m, k_{n}=k_{m} .
$$

We can assume

$$
k_{1}=k_{2},
$$

then by the definition of $\mathscr{G}$ we deduce that

$$
k_{3}=-2 k_{1} \text {. }
$$

On the other hand by (5.28) $k_{1},-2 k_{1} \in\left\{j_{1}, j_{2}, j_{3}\right\}$. Since by the definition of $\mathscr{G}$ we have $\sum_{i=1}^{3} j_{i}=0$ we conclude that necessarily

$$
\left\{j_{1}, j_{2}, j_{3}\right\}=\left\{k_{1}, k_{1},-2 k_{1}\right\} .
$$

On the other hand by (5.31), (5.32) we get

$$
\left\{k_{1}, k_{2}, k_{3}\right\}=\left\{k_{1}, k_{1},-2 k_{1}\right\}
$$

which in conjunction with (5.33) gives $\left\{k_{1}, k_{2}, k_{3}\right\}=\left\{j_{1}, j_{2}, j_{3}\right\}$. Hence we get a contradiction with the Hypothesis (5.8).

Lemma 5.4. - Let $m \geq 0$ be an integer and $p_{3}(u) \in \mathscr{P}_{3}(u)$ such that $\tilde{p}_{3}(u)=$ $u \partial_{x}^{m} u \partial_{x}^{m+1} u$. Then there exists $C>0$ such that

$$
\left\|f_{N}^{p_{3}}(u)-f_{M}^{p_{3}}(u)\right\|_{L^{2}\left(d \mu_{m+1}\right)} \leq \frac{C}{\sqrt{\min \{N, M\}}}, \forall N, M \in \mathbb{N} .
$$

Proof. - We assume for simplicity $p_{3}(u)=u \partial_{x}^{m} u \partial_{x}^{m+1} u$ (the general case can be treated in a similar way). Next we assume $N>M$ and we shall use the parametrization (1.3) with $k=2(m+1)$ to describe our probability space. Hence we get the following representation

$$
f_{N}^{p_{3}}(\varphi(\omega))-f_{M}^{p_{3}}(\varphi(\omega))=\sum_{(i, j, k) \in \mathscr{C}_{M}^{N}} \frac{1}{|i|^{m+1}} \frac{1}{|j|} \varphi_{i}(\omega) \varphi_{j}(\omega) \varphi_{k}(\omega)
$$

where

$$
\mathscr{G}_{M}^{N}=\{(i, j, k) \in \mathscr{G}|| i|,| j|,| k \mid \leq N \text { and } \max \{|i|,|j|,|k|\}>M\}
$$


and $\mathscr{G}$ is defined as in (5.7). By Lemma 5.3 we get:

$$
\begin{aligned}
& \left\|f_{N}^{p_{3}}(\varphi(\omega))-f_{M}^{p_{3}}(\varphi(\omega))\right\|_{L_{\omega}^{2}}^{2} \\
& \quad \leq \sum_{(i, j, k) \in \tilde{\mathscr{G}}_{M}^{N}}\left[\frac{1}{|i|^{m+1}}\left(\frac{1}{|j|}+\frac{1}{|k|}\right)+\frac{1}{|j|^{m+1}}\left(\frac{1}{|i|}+\frac{1}{|k|}\right)+\frac{1}{|k|^{m+1}}\left(\frac{1}{|i|}+\frac{1}{|j|}\right)\right]^{2}
\end{aligned}
$$

where

$$
\tilde{\mathscr{C}}_{M}^{N}=\left\{(i, j, k) \in \mathscr{G}_{M}^{N} \mid i \leq j \leq k\right\} .
$$

Next notice that the following elementary property holds

$$
\left.\tilde{\mathscr{G}}_{M}^{N} \subset\{(i, j, k) \in \mathscr{G} \mid \operatorname{Card}\{|i|,|j|,|k|\} \in[M / 2, N]\} \geq 2\right\}
$$

and hence we easily get:

$$
\begin{aligned}
\sum_{(i, j, k) \in \tilde{\mathscr{G}}_{M}^{N}}\left[\frac{1}{|i|^{m+1}}\left(\frac{1}{|j|}+\frac{1}{|k|}\right)+\frac{1}{|j|^{m+1}}\left(\frac{1}{|i|}\right.\right. & \left.\left.+\frac{1}{|k|}\right)+\frac{1}{|k|^{m+1}}\left(\frac{1}{|i|}+\frac{1}{|j|}\right)\right]^{2} \\
& \leq C \sum_{(l, n) \in \mathbb{N} \times \mathbb{N} n \geq \frac{M}{2}} \frac{1}{l^{2(m+1)} n^{2}} \leq \frac{C}{M} .
\end{aligned}
$$

In the next lemma the functions $h_{K}(u)$ are the ones defined in Proposition 5.2.

Lemma 5.5. - Let $m \geq 0$ be an integer. Then there exists $C>0$ such that

$$
\left\|h_{N}(u)-h_{M}(u)\right\|_{L^{2}\left(d \mu_{m+1}\right)} \leq \frac{C}{\sqrt{\min \{N, M\}}}, \forall N, M \in \mathbb{N} .
$$

Proof. - Notice that $\|\varphi(\omega)\|_{\dot{H}^{m+1 / 2}}^{2}=\sum_{n \in \mathbb{Z} \backslash\{0\}} \frac{1}{|n|}\left|\varphi_{n}(\omega)\right|^{2}$ where $\varphi(\omega)$ is defined as in (1.3) for $m=2(k+1)$. Hence the proof follows as in [14] (see Lemma 4.7).

Proof of Proposition 5.1. - In Lemma 5.4 we have proved (5.1) for $p=2$. The case $p>2$ follows by combining the estimate for $p=2$ with the Wiener Chaos in the same spirit as the paper [14] (see the proof of Lemma 4.3 in [14]). The estimate (5.2) follows by (5.1) in conjunction with Proposition 4.1.

We refer to [9] for a background on the estimates for the Wiener Chaos.

Proof of Proposition 5.2. - By combining Lemma 5.5 with the Wiener Chaos in the spirit of [14] we get (5.4) for any $p \geq 2$ (see the proof of Lemma 4.8 in [14]). Finally (5.5) follows by combining (5.4) with Proposition 4.1.

Arguing as in the proof of Proposition 5.1 and 5.2 we can prove the following result (that will be useful in the sequel to prove Theorem 1.1 in the special case $k=2$ ).

Proposition 5.6. - There exists $C>0$ such that

$$
\begin{gathered}
\mu_{1}\left\{u \in H^{1 / 2-\epsilon}|| E_{1 / 2}\left(\pi_{N} u\right)-\alpha_{N}-E_{1 / 2}\left(\pi_{M} u\right)+\alpha_{M} \mid>\lambda\right\} \\
\leq e^{-\frac{1}{C}(\lambda \sqrt{\min \{M, N\}})^{2 / 3}}
\end{gathered}
$$

and

$$
\mu_{1}\left\{u \in H^{1 / 2-\epsilon} \mid\left\|\pi_{N} u-\pi_{M} u\right\|_{L^{2}}^{2}>\lambda\right\} \leq e^{-\frac{1}{C}(\lambda \sqrt{\min \{M, N\}})}
$$

4 ${ }^{\mathrm{e}}$ SÉRIE - TOME 46 - 2013 - No 2 


$$
\forall M, N \in \mathbb{N}, \lambda>0 .
$$

Proof. - The proof of (5.36) follows the same argument as the proof of (5.2) and (5.5) (i.e., it follows by combining Lemma 5.3, with the Wiener Chaos and Proposition 4.1). By a similar argument we can prove

$$
\mu_{1}\left\{u \in H^{1 / 2-\epsilon}|| R_{1 / 2}\left(\pi_{N} u\right)-R_{1 / 2}\left(\pi_{M} u\right) \mid>\lambda\right\} \leq e^{-\frac{1}{C}(\lambda \sqrt{\min \{M, N\}})^{2 / 3}} .
$$

By combining this estimate with (5.5) (for $m=0$ ) we get (5.35).

\section{Proof of Theorem 1.1 for $k=2(m+1), m \geq 0$}

Along this section, when it is not better specified, we shall assume that $m \geq 0$ is a given integer. We recall the following notations to describe the energies preserved by the BenjaminOno flow:

$$
\begin{aligned}
E_{m+1}(u) & =\|u\|_{\dot{H}^{m+1}}^{2}+R_{m+1}(u) \\
E_{m+1 / 2}(u) & =\|u\|_{\dot{H}^{m+1 / 2}}^{2}+R_{m+1 / 2}(u) .
\end{aligned}
$$

We also introduce the following functions

$$
\begin{aligned}
& f_{N}: H^{m+1 / 2-\epsilon} \ni u \rightarrow R_{m+1}\left(\pi_{N} u\right) ; \\
& g_{N}: H^{m+1 / 2-\epsilon} \ni u \rightarrow E_{m+1 / 2}\left(\pi_{N} u\right)-\alpha_{N}
\end{aligned}
$$

(recall that $H^{m+1 / 2-\epsilon}$ is of full measure for $\mu_{m+1}$ ). Notice that we can write the identity

$$
g_{N}(u)-h_{N}(u)=R_{m+1 / 2}\left(\pi_{N} u\right)
$$

where $h_{N}(u)$ is defined as in Proposition 5.2. For every $p_{j}(u) \in \mathscr{P}_{j}(u)$ we introduce

$$
f_{N}^{p_{j}}: H^{m+1 / 2-\epsilon} \ni u \rightarrow \int p_{j}\left(\pi_{N} u\right) d x \in \mathbb{R} .
$$

Next we split the proof of Theorem 1.1 (in the case $k=2(m+1)$ ) in several propositions.

Proposition 6.1. - Let $m \geq 0$ be an integer and $\psi \in C_{c}(\mathbb{R})$ be given. Then there exist two functions $\bar{h}(u), \bar{f}(u)$ measurable with respect to $\mu_{m+1}$ such that:

$$
\begin{aligned}
& \left.|\bar{h}(u)|,|\bar{f}(u)|<\infty, \text { a.e. (w.r.t. } \mu_{m+1}\right) u \in H^{m+1 / 2-\epsilon} \\
& \prod_{j=0}^{2 m} \psi\left(E_{j / 2}\left(\pi_{N} u\right)\right) \psi\left(E_{m+1 / 2}\left(\pi_{N} u\right)-\alpha_{N}\right) e^{-R_{m+1}\left(\pi_{N} u\right)}
\end{aligned}
$$

converges in measure to

$$
\prod_{j=0}^{2 m} \psi\left(E_{j / 2}(u)\right) \psi\left(\bar{h}(u)+R_{m+1 / 2}(u)\right) e^{-\bar{f}(u)} .
$$

Moreover

$$
\left.\left|E_{j / 2}(u)\right|,\left|R_{m+1 / 2}(u)\right|<\infty \text {, a.e. (w.r.t. } \mu_{m+1}\right) u \in H^{m+1 / 2-\epsilon} .
$$

The proof of (6.4) follows by (6.12) and (6.13) in Lemma 6.3. Hence Proposition 6.1 follows by Lemmas 6.3, 6.4 in conjunction with the following proposition. 
Proposition 6.2. - Let $m \geq 0$ be an integer and $\psi \in C_{c}(\mathbb{R})$ be given. There exist $\bar{f}(u), \bar{h}(u)$ measurable functions with respect to $\mu_{m+1}$ such that:

$$
\begin{gathered}
\left.|\bar{h}(u)|,|\bar{f}(u)|<\infty \text {, a.e. (w.r.t. } \mu_{m+1}\right) u \in H^{m+1 / 2-\epsilon} ; \\
R_{m+1}\left(\pi_{N} u\right) \text { converges in measure w.r.t. } \mu_{m+1} \text { to } \bar{f}(u) ; \\
\lim _{N \rightarrow \infty}\left\|\psi\left(g_{N}(u)\right)-\psi\left(\bar{h}(u)+R_{m+1 / 2}(u)\right)\right\|_{L^{q}\left(d \mu_{m+1}\right)}=0, \forall q \in[1, \infty) .
\end{gathered}
$$

Moreover we have

$$
\lim _{N \rightarrow \infty}\left\|\psi\left(E_{j / 2}\left(\pi_{N} u\right)\right)-\psi\left(E_{j / 2}(u)\right)\right\|_{L^{q}\left(d \mu_{m+1}\right)}=0, \forall q \in[1, \infty), j=0, \ldots, 2 m .
$$

First we prove the following lemma.

Lemma 6.3. - Let $m \geq 1$ be an integer; then the following limits exist:

$$
\left.\lim _{N \rightarrow \infty} f_{N}^{p_{j}}(u)=\int p_{j}(u) d x \in \mathbb{R} \text {, a.e. (w.r.t. } \mu_{m+1}\right) u \in H^{m+1 / 2-\epsilon}
$$

provided that

$$
j \geq 3, \tilde{p}_{j}(u)=\prod_{i=1}^{j} \partial_{x}^{\alpha_{i}} u \text { with } 0 \leq \sum_{i=1}^{j} \alpha_{i} \leq 2 m
$$

or

$$
j=3, \tilde{p}_{3}(u)=\prod_{i=1}^{3} \partial_{x}^{\alpha_{i}} u \text { with } 0 \leq \sum_{i=1}^{3} \alpha_{i}=2 m+1 \text { and } \min _{i=1,2,3} \alpha_{i} \geq 1 .
$$

In particular

$$
\left.\lim _{N \rightarrow \infty} R_{m+1 / 2}\left(\pi_{N} u\right)=R_{m+1 / 2}(u) \in \mathbb{R} \text { a.e. (w.r.t. } \mu_{m+1}\right) u \in H^{m+1 / 2-\epsilon} .
$$

Moreover we have

$$
\left|E_{j / 2}(u)\right|<\infty, \forall u \in H^{m+1 / 2-\epsilon}, j=0, \ldots, 2 m .
$$

Proof. - We assume for simplicity $p_{j}(u)=\tilde{p}_{j}(u)$ (the general case can be treated by a similar argument).

The proof of (6.9) (under the Hypothesis (6.10)) follows by Lemma 3.6.

Concerning the proof of (6.9), under the assumption (6.11), we notice that by integration by parts we can assume

$$
1 \leq \alpha_{1} \leq \alpha_{2} \leq \alpha_{3} \leq m
$$

Hence we get

$$
\left|\int p_{3}(u) d x\right| \leq C\|u\|_{H^{m}}^{2}\left\|\partial_{x}^{\alpha_{1}} u\right\|_{L^{\infty}} \leq C\|u\|_{H^{m}}^{2}\|u\|_{W^{m+\epsilon, p}}
$$

where we have used the Sobolev embedding $W^{\epsilon, p} \subset L^{\infty}$ provided that $\epsilon p>1$. On the other hand by a suitable version of Proposition 4.2 (where we replace $\|\cdot\|_{W^{\epsilon, p}}$ by $\|\cdot\|_{W^{m+\epsilon, p}}$ ) we get $u \in W^{m+\epsilon, p}$, a.e. (w.r.t. $\mu_{m+1}$ ) $u \in H^{m+1 / 2-\epsilon}$ and hence (6.9) follows.

The proof of (6.12) follows by combining the structure of $E_{m+1 / 2}$ (see (2.5)) with (6.9) (under the assumption (6.10)). The proof of (6.13) follows by a similar argument.

The next result is a suitable version of the previous lemma in the case $m=0$. 
Lemma 6.4. - The following limits exist:

$$
\begin{aligned}
& \lim _{N \rightarrow \infty} R_{1 / 2}\left(\pi_{N} u\right)=R_{1 / 2}(u) \in \mathbb{R}, \forall u \in H^{1 / 2-\epsilon} ; \\
& \lim _{N \rightarrow \infty} \int\left(\pi_{N} u\right)^{4} d x=\int u^{4} d x, \forall u \in H^{1 / 2-\epsilon} .
\end{aligned}
$$

Proof. - By looking at the explicit structure on $E_{1 / 2}$ (see the introduction) we get $R_{1 / 2}(u)=\frac{1}{3} \int u^{3} d x$. On the other hand by the Sobolev embedding $H^{1 / 2-\epsilon} \subset L^{3}$ we get $u \in L^{3}$, and hence (6.14) follows. By a similar argument we deduce (6.15).

Proof of Proposition 6.2. - By Proposition 5.1 and 5.2 there exist two functions $\tilde{f}, \bar{h} \in \cap_{q=1}^{\infty} L^{q}\left(d \mu_{m+1}\right)$ such that:

$$
\begin{aligned}
\lim _{N \rightarrow \infty}\left\|f_{N}^{p_{3}}(u)-\tilde{f}(u)\right\|_{L^{q}\left(d \mu_{m+1}\right)} & =0\left(\text { provided that } \tilde{p}_{3}(u)=u \partial_{x}^{m} u \partial_{x}^{m+1} u\right) ; \\
\lim _{N \rightarrow \infty}\left\|h_{N}(u)-\bar{h}(u)\right\|_{L^{q}\left(d \mu_{m+1}\right)} & =0 .
\end{aligned}
$$

Proof of (6.6). - If $m=0$ then it follows by (6.15), (6.16) and by looking at the explicit structure of $E_{1}$ (see the introduction).

If $m \geq 1$ then it follows by combining (6.9) (under both assumptions (6.10) and (6.11)), (6.16) and the algebraic structure of $R_{m+1}(u)$ (see (2.4)).

Proof of (6.7). - It is sufficient to prove that for every sequence $N_{k}$ there is a subsequence $N_{k_{h}}$ such that

$$
\lim _{h \rightarrow \infty}\left\|\psi\left(g_{N_{k_{h}}}(u)\right)-\psi\left(\bar{h}(u)+R_{m+1 / 2}(u)\right)\right\|_{L^{q}\left(d \mu_{m+1}\right)}=0 .
$$

Notice that by combining (6.12) (when $m \geq 1$ ) and (6.14) (when $m=0$ ) with (6.17) we get a subsequence $N_{k}$ such that

$$
\left.\lim _{k \rightarrow \infty}\left(h_{N_{k}}(u)+R_{m+1 / 2}\left(\pi_{N_{k}} u\right)\right)=\bar{h}(u)+R_{m+1 / 2}(u) \text { a.e. (w.r.t. } \mu_{m+1}\right) u \in H^{m+1 / 2-\epsilon} .
$$

Since $\sup _{u \in H^{m+1 / 2-\epsilon}}\left|\psi\left(g_{N_{k}}(u)\right)\right| \leq \sup \psi$ and $d \mu_{m+1}\left(H^{m+1 / 2-\epsilon}\right)=1$ we can apply the dominated convergence theorem to get (6.18).

Proof of (6.8). - If $m \geq 1$ then we combine (6.9) (under the assumption (6.10)) with (2.4) and (2.5) in order to get $E_{j / 2}\left(\pi_{N} u\right) \rightarrow E_{j / 2}(u)$, a.e. (w.r.t. $\left.\mu_{m+1}\right) u \in H^{m+1 / 2-\epsilon}$ for $j=0, . ., 2 m$. Hence the proof for $m \geq 1$ can be concluded as in (6.7).

The case $m=0$ is simpler since we have $\mu_{1}\left(L^{2}\right)=1$ and hence

$$
\left.\left\|\pi_{N} u\right\|_{L^{2}} \rightarrow\|u\|_{L^{2}} \text {, a.e. (w.r.t. } \mu_{1}\right) u \in L^{2} \text {. }
$$

The proof follows as above.

The next proposition allows us to deduce that the limit functions constructed in (6.3) belong to $L^{q}\left(d \mu_{m+1}\right)$.

Proposition 6.5. - Let $m \geq 0$ and $\psi \in C_{c}(\mathbb{R})$ be given. For every $q \in[1, \infty)$ we have

$$
\sup _{N}\left\|\prod_{j=0}^{2 m} \psi\left(E_{j / 2}\left(\pi_{N} u\right)\right) \psi\left(E_{m+1 / 2}\left(\pi_{N} u\right)-\alpha_{N}\right) e^{-R_{m+1}\left(\pi_{N} u\right)}\right\|_{L^{q}\left(d \mu_{m+1}\right)}<\infty .
$$


Lemma 6.6. - Let $m \geq 0$ be an integer and $p_{3}(u) \in \mathscr{P}_{3}(u)$ such that $\tilde{p}_{3}(u)=u \partial_{x}^{m} u \partial_{x}^{m+1} u$. For every $R>0$ there exists $C=C(R)>0$ such that

$$
\mu_{m+1}\left\{u \in H^{m+1 / 2-\epsilon}|| f_{N}^{p_{3}}(u)|>\lambda,| h_{N}(u) \mid<R\right\} \leq C e^{-\frac{\lambda^{2}}{C \alpha_{N}^{2}}} \forall N \in \mathbb{N}, \lambda>0 .
$$

Proof. - We fix $0<\epsilon<\frac{1}{2}$ and $1<p<\infty$ such that $\epsilon p>1$. Then by Lemma 3.4 we get

$$
\left|f_{N}^{p_{3}}(u)\right| \leq C\left\|\pi_{N} u\right\|_{H^{m+1 / 2}}^{2}\left\|\pi_{N} u\right\|_{W^{\epsilon, p}}
$$

and hence

$$
\left|f_{N}^{p_{3}}(u)\right| \leq C\left(\alpha_{N}+R\right)\left\|\pi_{N} u\right\|_{W^{\epsilon, p}} \forall u \in\left\{u \in H^{m+1 / 2-\epsilon}|| h_{N}(u) \mid<R\right\} .
$$

The proof follows by Proposition 4.2 (in fact notice that the same proof of Proposition 4.2 works in the case when the vector $\varphi(\omega)$ is replaced by $\pi_{N} \varphi(\omega)$ with uniform bounds that do not depend on $N$ ).

Next we present a modified version of Lemma 6.6 that will be useful to prove Theorem 1.1 for $k=2$ (i.e., $m=0$ following the notation introduced in this section).

REMARK 6.7. - Indeed the main difference between the case $m=0$ and $m>0$ is that Proposition 3.1 is not available for $m=0$.

Lemma 6.8. - Let $p_{3}(u) \in \mathscr{P}_{3}(u)$ be such that $\tilde{p}_{3}(u)=u^{2} \partial_{x} u$. For every $R>0$ there exists $C=C(R)>0$ such that

$$
\begin{aligned}
\mu_{1}\left\{u \in H^{1 / 2-\epsilon}|| f_{N}^{p_{3}}(u) \mid\right. & \left.>\lambda,\left\|\pi_{N} u\right\|_{L^{2}}<R,\left|E_{1 / 2}\left(\pi_{N} u\right)-\alpha_{N}\right|<R\right\} \\
& \leq C e^{-\frac{\lambda^{2}}{C \alpha_{N}^{2}}}, \forall N \in \mathbb{N}, \lambda>0 .
\end{aligned}
$$

Proof. - First notice that due to (3.11) we have the following estimate:

$$
\begin{aligned}
\left\|\pi_{N} u\right\|_{H^{1 / 2}}^{2} & \leq\left|R_{1 / 2}\left(\pi_{N} u\right)\right|+\left|E_{1 / 2}\left(\pi_{N} u\right)\right| \\
& \leq C R^{3 / 2}\left\|\pi_{N} u\right\|_{H^{\frac{1}{2}}}^{3 / 2}+\alpha_{N}+R \\
\forall u & \in\left\{u \in H^{1 / 2-\epsilon}\left|\left\|\pi_{N} u\right\|_{L^{2}}<R,\right| E_{1 / 2}\left(\pi_{N} u\right)-\alpha_{N} \mid<R\right\} .
\end{aligned}
$$

The estimate above implies

$$
\begin{gathered}
\left\|\pi_{N} u\right\|_{H^{\frac{1}{2}}}^{2} \leq C\left(\alpha_{N}+1\right) \\
\forall u \in\left\{u \in H^{1 / 2-\epsilon}\left|\left\|\pi_{N} u\right\|_{L^{2}}<R,\right| E_{1 / 2}\left(\pi_{N} u\right)-\alpha_{N} \mid<R\right\}
\end{gathered}
$$

where $C>0$ is a suitable constant. By combining Lemma 3.4 with (6.22) we get

$$
\begin{aligned}
\left|\int p_{3}\left(\pi_{N}(u)\right) d x\right| & \leq C\left\|\pi_{N} u\right\|_{H^{1 / 2}}^{2}\left\|\pi_{N} u\right\|_{W^{\epsilon, p}} \\
& \leq C\left(\alpha_{N}+1\right)\left\|\pi_{N} u\right\|_{W^{\epsilon, p}}
\end{aligned}
$$

The proof can be concluded as in Lemma 6.3.

In the sequel the sets $A_{M, N}^{p, \lambda}$ and $B_{M, N}^{\lambda}$ are the ones introduced in (5.3) and (5.6). 
Lemma 6.9. - Let $m \geq 0$ be an integer and $p_{3}(u) \in \mathscr{P}_{3}(u)$ such that $\tilde{p}_{3}(u)=u \partial_{x}^{m} u \partial_{x}^{m+1} u$. Then

$$
\begin{array}{r}
\mu_{m+1}\left\{u \in H^{m+1 / 2-\epsilon}|| f_{N}^{p_{3}}(u)|>\lambda,| h_{N}(u) \mid<R\right\} \leq d \mu_{m+1}\left(B_{M, N}^{S}\right) \\
+\mu_{m+1}\left\{u \in H^{m+1 / 2-\epsilon}|| f_{M}^{p_{3}}(u)\left|>\frac{\lambda}{2},\right| h_{M}(u) \mid<R+S\right\}+d \mu_{m+1}\left(A_{M, N}^{p_{3}, \frac{\lambda}{2}}\right), \\
\forall M, N, \lambda, R, S .
\end{array}
$$

Proof. - We have the following elementary estimates:

$$
\begin{aligned}
\mu_{m+1}\{u & \left.\in H^{m+1 / 2-\epsilon}|| f_{N}^{p_{3}}(u)|>\lambda,| h_{N}(u) \mid<R\right\} \\
\leq & \mu_{m+1}\left\{u \in H^{m+1 / 2-\epsilon}|| f_{M}^{p_{3}}(u)\left|>\frac{\lambda}{2},\right| h_{N}(u) \mid<R\right\}+\mu_{m+1}\left(A_{M, N}^{p_{3}, \frac{\lambda}{2}}\right) \\
\leq & \mu_{m+1}\left\{u \in H^{m+1 / 2-\epsilon} \backslash B_{M, N}^{S}|| f_{M}^{p_{3}}(u)\left|>\frac{\lambda}{2},\right| h_{N}(u) \mid<R\right\}+\mu_{m+1}\left(B_{M, N}^{S}\right) \\
& +\mu_{m+1}\left(A_{M, N}^{p_{3}, \frac{\lambda}{2}}\right) .
\end{aligned}
$$

On the other hand

$$
\left\{u \in H^{m+1 / 2-\epsilon} \backslash B_{M, N}^{S}|| h_{N}(u) \mid<R\right\} \subset\left\{u \in H^{m+1 / 2-\epsilon}|| h_{M}(u) \mid<R+S\right\}
$$

and hence we get (6.23).

Next we propose a modified version of Lemma 6.9 that will be useful to prove Theorem 1.1 for $k=2$ (i.e., $m=0$ following the notation introduced in this section). See Remark 6.7 to understand the difference between the case $m=0$ and $m>0$.

Lemma 6.10. - Let $p_{3}(u) \in \mathscr{P}_{3}(u)$ such that $\tilde{p}_{3}(u)=u^{2} \partial_{x} u$. Then

$$
\begin{aligned}
\mu_{1}\{u & \left.\in H^{1 / 2-\epsilon}|| f_{N}^{p_{3}}(u)\left|>\lambda,\left\|\pi_{N} u\right\|_{L^{2}}<R,\right| E_{1 / 2}\left(\pi_{N} u\right)-\alpha_{N} \mid<R\right\} \\
\leq & \mu_{1}\left\{u \in H^{1 / 2-\epsilon}|| E_{1 / 2}\left(\pi_{N} u\right)-\alpha_{N}-E_{1 / 2}\left(\pi_{M} u\right)+\alpha_{M} \mid>S\right\} \\
& +\mu_{1}\left\{u \in H^{\frac{1}{2}-\epsilon}|| f_{M}^{p_{3}}(u)\left|>\frac{\lambda}{2},\left\|\pi_{M} u\right\|_{L^{2}}<R+S,\right| E_{1 / 2}\left(\pi_{M} u\right)-\alpha_{M} \mid<R+S\right\} \\
& +\mu_{1}\left(A_{M, N}^{p_{3}, \frac{\lambda}{2}}\right)+\mu_{1}\left\{u \in H^{1 / 2-\epsilon} \mid\left\|\pi_{M} u-\pi_{N} u\right\|_{L^{2}}>S\right\}, \forall M, N, \lambda, R, S .
\end{aligned}
$$

Proof. - It is similar to the proof of Lemma 6.9.

Proof of Proposition 6.5. - We have to prove (6.19).

Claim. - It is sufficient to prove (6.19) with $f_{N}$ replaced by $f_{N}^{p_{3}}$ where

$$
\tilde{p}_{3}(u)=u \partial_{x}^{m} u \partial_{x}^{m+1} u \text {. }
$$

To prove the claim first notice that due to the factor $\prod_{j=0}^{2 m} \psi\left(E_{j / 2}\left(\pi_{N} u\right)\right)$ in (6.19) and due to (3.10), we deduce that the $L^{q}\left(d \mu_{m+1}\right)$ norm in (6.19) can be computed on a sub-region $\Omega_{N} \subset H^{m+1 / 2-\epsilon}$ such that

$$
\left.\Omega_{N}=\left\{u \in H^{m+1 / 2-\epsilon} \mid\left\|\pi_{N} u\right\|_{H^{m}}\right\}<C\right\}
$$

with $C$ that does not depend on $N$. Next we prove the claim. 
First case: $m \geq 2$.

By combining Lemma 3.5 and Lemma 3.6, and by looking at the structure of $E_{m+1}$ in (2.4) we deduce that

$$
\sup _{N}\left\|R_{m+1}\left(\pi_{N} u\right)-\sum_{\substack{p_{3}(u) \in \mathscr{\mathcal { P }}_{3}(u) \text { s.t. } \\ \tilde{p}_{3}(u)=u \partial_{x}^{m} u \partial_{x}^{m+1} u}} c_{2(m+1)}(p) \int p_{3}\left(\pi_{N} u\right) d x\right\|_{L^{\infty}\left(\Omega_{N}\right)}<\infty .
$$

This implies the claim for $m \geq 2$.

Second case: $m=0$.

In the case $m=0$ we have

$$
f_{N}(u)=\frac{3}{4} \int\left(\pi_{N} u\right)^{2} H\left(\pi_{N} \partial_{x} u\right) d x+\frac{1}{8} \int\left(\pi_{N} u\right)^{4} d x
$$

and hence we get the claim due to the positivity of the last term.

Third case: $m=1$.

By looking at the structure of $E_{2}$ and by using Lemma 3.6 we deduce that the claim follows provided that we prove

$$
\sup _{N}\left\|e^{q\left|f^{p}\left(\pi_{N} u\right)\right|}\right\|_{L^{1}\left(\Omega_{N}, d \mu_{2}\right)}<\infty
$$

where $\Omega_{N}$ is defined in (6.25) and $p(u) \in \mathscr{P}_{3}(u)$ is such that $\tilde{p}(u)=\left(\partial_{x} u\right)^{3}$. For simplicity we treat the case $p(u)=\left(\partial_{x} u\right)^{3}$ (the general case can be treated in a similar way). We have the following estimate

$$
\left|f^{p}\left(\pi_{N} u\right)\right| \leq C\left\|\pi_{N} u\right\|_{H^{1}}^{2}\left\|\partial_{x} \pi_{N} u\right\|_{L^{\infty}} \leq C\left\|\pi_{N} u\right\|_{W^{1+\epsilon, p}}
$$

provided that $u \in \Omega_{N}$ and $\epsilon p>1$. Hence we get

$$
\left\{u \in \Omega_{N}|| f^{p}\left(\pi_{N} u\right) \mid>\lambda\right\} \subset\left\{u \in \Omega_{N} \mid\left\|\pi_{N}(u)\right\|_{W^{1+\epsilon, p}}>\lambda C^{-1}\right\}
$$

which in turn implies

$$
\mu_{2}\left\{u \in \Omega_{N}|| f^{p}\left(\pi_{N} u\right) \mid>\lambda\right\} \leq C e^{-\frac{\lambda^{2}}{C}}
$$

where we have used an adapted version of Proposition 4.2 (i.e., we choose $\varphi(\omega)$ as in (1.3) with $k=4$ and the norm $W^{\epsilon, p}$ is replaced by $\left.W^{1+\epsilon, p}\right)$. As a consequence we get

$$
\int_{\Omega_{N}} e^{q\left|f^{p}\left(\pi_{N} u\right)\right|} d \mu_{2} \leq \int_{0}^{\infty} e^{q \lambda} d \mu_{2}\left\{u \in \Omega_{N}|| f^{p}\left(\pi_{N} u\right) \mid>\lambda\right\} d \lambda<\infty .
$$

Next we shall prove (6.19) where $f_{N}$ is replaced by $f_{N}^{p_{3}}$ with $p_{3}(u)$ that satisfy (6.24), and it will complete the proof.

We split the proof in two subcases.

First case: $m>0$.

Since $\psi$ is compactly supported there exists $R>0$ such that

$$
\left.0 \leq \psi\left(E_{j / 2}\left(\pi_{N} u\right)\right) \leq \chi_{\left\{u \in H^{m+1 / 2-\epsilon}|| E_{j / 2}\left(\pi_{N} u\right) \mid<R\right\}} \text { a.e. (w.r.t. } \mu_{m+1}\right) u, \forall j=0, \ldots, 2 m
$$

and also

$$
\left.0 \leq \psi\left(g_{N}(u)\right) \leq \chi_{\left\{u \in H^{m+1 / 2-\epsilon}|| g_{N}(u) \mid<R\right\}}, \text { a.e. (w.r.t. } \mu_{m+1}\right) u
$$


where $\chi_{A}$ denotes in general the characteristic function of $A$. In particular $0 \leq \prod_{j=0}^{2 m} \psi\left(E_{j / 2}(u)\right) \psi\left(g_{N}(u)\right) \leq \chi_{\bigcap_{j=0}^{2 m}\left\{u \in H^{m+1 / 2-\epsilon}|| E_{j / 2}\left(\pi_{N} u\right) \mid<R\right\} \cap\left\{u \in H^{m+1 / 2-\epsilon}|| g_{N}(u) \mid<R\right\}}$ which due to Proposition 3.1 implies

$$
0 \leq \prod_{j=0}^{2 m} \psi\left(E_{j / 2}\left(\pi_{N} u\right)\right) \psi\left(g_{N}(u)\right) \leq \chi_{\left\{u \in H^{m+1 / 2-\epsilon}|| h_{N}(u) \mid<R\right\}}
$$

for a suitable $R>0$ that can be different from the previous one. Hence it is sufficient to prove

$$
\sup _{N} \int_{\left\{u \in H^{m+1 / 2-\epsilon}|| h_{N}(u) \mid<R\right\}} e^{q\left|f_{N}^{p_{3}}(u)\right|} d \mu_{m+1}<\infty, \forall N \in \mathbb{N}
$$

where $\tilde{p}_{3}(u)$ satisfies (6.24). The estimate above is equivalent to:

$$
\sup _{N} \int_{0}^{\infty} \mu_{m+1}\left\{u \in H^{m+1 / 2-\epsilon}|| f_{N}^{p_{3}}(u)|>\lambda,| h_{N}(u) \mid<R\right\} e^{q \lambda} d \lambda<\infty .
$$

In turn it follows by the following ones:

$$
\sup _{N} \int_{\sqrt{N}}^{\infty} \mu_{m+1}\left\{u \in H^{m+1 / 2-\epsilon}|| f_{N}^{p_{3}}(u)|>\lambda,| h_{N}(u) \mid<R\right\} e^{q \lambda} d \lambda<\infty
$$

and

$$
\sup _{N} \int_{0}^{\sqrt{N}} \mu_{m+1}\left\{u \in H^{m+1 / 2-\epsilon}|| f_{N}^{p_{3}}(u)|>\lambda,| h_{N}(u) \mid<R\right\} e^{q \lambda} d \lambda<\infty .
$$

By (6.20) we get:

$$
\begin{aligned}
\sup _{N} & \int_{\sqrt{N}}^{\infty} \mu_{m+1}\left\{u \in H^{m+1 / 2-\epsilon}|| f_{N}^{p_{3}}(u)|>\lambda,| h_{N}(u) \mid<R\right\} e^{q \lambda} d \lambda \\
& \leq \sup _{N} \int_{\sqrt{N}}^{\infty} C e^{-\frac{\lambda^{2}}{C \alpha_{N}^{2}}} e^{q \lambda} d \lambda=C \sup _{N} e^{\frac{C q^{2} \alpha_{N}^{2}}{4}} \int_{\sqrt{N}}^{\infty} e^{-\left(\frac{\lambda}{\sqrt{C} \alpha_{N}}-\frac{q}{2} \sqrt{C} \alpha_{N}\right)^{2}} d \lambda \\
& =C \sup _{N} e^{\frac{C q^{2} \alpha_{N}^{2}}{4}} \int_{\frac{\sqrt{N}}{\sqrt{C} \alpha_{N}}-\frac{q}{2} \sqrt{C} \alpha_{N}}^{\infty} e^{-\lambda^{2} \sqrt{C} \alpha_{N} d \lambda}
\end{aligned}
$$

which due to the bound $e^{-r^{2}}<e^{-r}$ for every $r>1$ can be estimated by

$$
\cdots \leq C^{\frac{3}{2}} \sup _{N}\left(\alpha_{N} e^{\frac{C q^{2} \alpha_{N}^{2}}{4}} e^{-\frac{\sqrt{N}}{\sqrt{C} \alpha_{N}}+\frac{q}{2} \sqrt{C} \alpha_{N}}\right)
$$

which implies (6.27).

In order to prove (6.28) we use (6.23) where we fix $M=[\lambda]^{2}$ (here $[\lambda]$ is the integer part of $\lambda$ ) and $S>0$ will be chosen later in a suitable way. By recalling also (5.2), (5.5) and (6.20) we get:

$$
\begin{aligned}
& \int_{0}^{\sqrt{N}} \mu_{m+1}\left\{u \in H^{m+1 / 2-\epsilon}|| f_{N}^{p_{3}}(u)|>\lambda,| h_{N}(u) \mid<R\right\} e^{q \lambda} d \lambda \\
& \leq \int_{0}^{\sqrt{N}} C e^{-\frac{\lambda^{2}}{C \alpha_{[\lambda]^{2}}^{2}}+q \lambda}+C\left(e^{-\frac{[\lambda] S}{C}+q \lambda}+e^{-\frac{1}{C}\left(\frac{\lambda}{2}[\lambda]\right)^{\frac{2}{3}}+q \lambda}\right) d \lambda
\end{aligned}
$$


where $C>0$ are uniform constant that can change at each step. Notice that if we choose $S$ large enough compared with $q$ then

$$
\begin{aligned}
& \sup _{N} \int_{0}^{\sqrt{N}} C e^{-\frac{\lambda^{2}}{C \alpha_{[\lambda]^{2}}^{2}}+q \lambda}+C\left(e^{-\frac{[\lambda] S}{C}+q \lambda}+e^{-\frac{1}{C}\left(\frac{\lambda}{2}[\lambda]\right)^{\frac{2}{3}}+q \lambda}\right) d \lambda \\
& \leq \int_{0}^{\infty} C e^{-\frac{\lambda^{2}}{C \alpha_{[\lambda]^{2}}^{2}}+q \lambda}+C\left(e^{-\frac{[\lambda] S}{C}+q \lambda}+e^{-\frac{1}{C}\left(\frac{\lambda}{2}[\lambda]\right)^{\frac{2}{3}}+q \lambda}\right) d \lambda<\infty
\end{aligned}
$$

which implies (6.28).

Second case: $m=0$.

The main difference with the case $m>0$ is that we cannot apply Proposition 3.1. Due to the cut-off function $\psi$ it is sufficient to prove

$$
\sup _{N} \int_{\left\{u \in H^{1 / 2-\epsilon}|| \pi_{N} u \|_{L^{2}}<R,\left|E_{1 / 2}\left(\pi_{N} u\right)-\alpha_{N}\right|<R\right\}} e^{q\left|f_{N}^{p_{3}}(u)\right|} d \mu_{1}<\infty, \forall N \in \mathbb{N}
$$

where $\tilde{p}_{3}(u)$ satisfies $\tilde{p}_{3}(u)=u^{2} \partial_{x} u$ and $R>0$ is a suitable constant. The estimate above is equivalent to:

$$
\sup _{N} \int_{0}^{\infty} \mu_{1}\left(\mathscr{C}_{\lambda, N}\right) e^{q \lambda} d \lambda<\infty
$$

where

$$
\mathscr{C}_{\lambda, N}=\left\{u \in H^{1 / 2-\epsilon}|| f_{N}^{p_{3}}(u)\left|>\lambda,\left\|\pi_{N} u\right\|_{L^{2}}<R,\right| E_{1 / 2}\left(\pi_{N} u\right)-\alpha_{N} \mid<R\right\}
$$

and in turn it follows by the following ones:

$$
\sup _{N} \int_{N^{\beta}}^{\infty} \mu_{1}\left(\mathscr{C}_{\lambda, N}\right) e^{q \lambda} d \lambda<\infty
$$

and

$$
\sup _{N} \int_{0}^{N^{\beta}} \mu_{1}\left(\mathscr{C}_{\lambda, N}\right) e^{q \lambda} d \lambda<\infty
$$

where $\beta>0$ will be fixed later. By Lemma 6.8 we get:

$$
\begin{aligned}
\sup _{N} \int_{N^{\beta}}^{\infty} \mu_{1}\left(\mathscr{C}_{\lambda, N}\right) e^{q \lambda} d \lambda & \leq \sup _{N} \int_{N^{\beta}}^{\infty} C e^{-\frac{\lambda^{2}}{C \alpha_{N}^{2}}} e^{q \lambda} d \lambda=C \sup _{N} e^{\frac{C q^{2} \alpha_{N}^{2}}{4}} \int_{N^{\beta}}^{\infty} e^{-\left(\frac{\lambda}{\sqrt{C} \alpha_{N}}-\frac{q}{2} \sqrt{C} \alpha_{N}\right)^{2}} d \lambda \\
& =C \sup _{N} e^{\frac{C q^{2} \alpha_{N}^{2}}{4}} \int_{\frac{N^{\beta}}{\sqrt{C} \alpha_{N}}-\frac{q}{2} \sqrt{C} \alpha_{N}}^{\infty} e^{-\lambda^{2} \sqrt{C} \alpha_{N} d \lambda}
\end{aligned}
$$

which due to the bound $e^{-r^{2}}<e^{-r}$ for every $r>1$ can be estimated by

$$
\cdots \leq C^{\frac{3}{2}} \sup _{N}\left(\alpha_{N} e^{\frac{C q^{2} \alpha_{N}^{2}}{4}} e^{-\frac{N^{\beta}}{\sqrt{C} \alpha_{N}}+\frac{q}{2} \sqrt{C} \alpha_{N}}\right)
$$

which implies (6.29) for every $\beta>0$.

4 ${ }^{\text {e }}$ SÉRIE - TOME $46-2013$ - No 2 
In order to prove (6.30) we use Lemma 6.10 where we fix $M=[\lambda]^{1 / \beta}$ (here $[\lambda]$ is the integer part of $\lambda$ ) and $S=1$. By recalling also (5.2), (5.35), (5.36) and (6.21) we get

$$
\begin{aligned}
& \sup _{N} \int_{0}^{N^{\beta}} \mu_{1}\left(\mathscr{C}_{\lambda, N}\right) e^{q \lambda} d \lambda \leq \sup _{N} \int_{0}^{N^{\beta}}\left[C e^{-\frac{\lambda^{2}}{C^{\prime} \alpha^{2}}[\lambda]^{1 / \beta}}+q \lambda\right. \\
& \left.+C\left(e^{-\frac{1}{C}\left(\frac{\lambda}{2}[\lambda]^{1 /(2 \beta)}\right)^{2 / 3}+q \lambda}+e^{-\frac{1}{C}\left(\lambda[\lambda]^{1 /(2 \beta)}\right)+q \lambda}\right)\right] d \lambda
\end{aligned}
$$

where $C>0$ denotes uniform constants which can change at each step. Notice that if we choose $\beta<1$ then we can continue the estimate as follows:

$$
\cdots \leq \sup _{N} \int_{0}^{\infty} e^{-C \lambda^{1+\epsilon_{0}}+q \lambda} d \lambda<\infty
$$

for suitable $C, \epsilon_{0}>0$. Hence we get (6.30).

Proposition 6.11. - Let $\bar{f}(u), \bar{h}(u)$ be as in Proposition 6.1 and $\chi_{R}$ as in Theorem 1.1. Then

$$
\left.\bigcup_{R>0} \operatorname{supp}\left(\prod_{j=0}^{2 m} \chi_{R}\left(E_{j / 2}(u)\right) \chi_{R}(\bar{h}(u))+R_{m+1 / 2}(u)\right) e^{-\bar{f}(u)}\right)=\operatorname{supp}\left(\mu_{m+1}\right) .
$$

Proof. - Due to (6.2) and (6.4) we get

$$
\mu_{m+1}\left\{u \in H^{m+1 / 2-\epsilon}|| \bar{h}(u) \mid=\infty\right\}=\mu_{m+1}\left\{u \in H^{m+1 / 2-\epsilon}|| R_{m+1 / 2}(u) \mid=\infty\right\}=0
$$

and

$$
\mu_{m+1}\left\{u \in H^{m+1 / 2-\epsilon} \mid e^{-\bar{f}(u)}=0\right\}=0 .
$$

Moreover by (6.4) we also get

$$
\mu_{m+1}\left\{u \in H^{m+1 / 2-\epsilon}|| E_{j / 2}(u) \mid=\infty\right\}=0, \forall j=0, \ldots, 2 m .
$$

As a consequence of (6.31) and (6.33), and by noticing that $\chi_{R}(t) \rightarrow 1$ as $R \rightarrow \infty$, we deduce:

$$
\left.\lim _{R \rightarrow \infty} \prod_{j=0}^{2 m} \chi_{R}\left(E_{j / 2}(u)\right) \chi_{R}\left(\bar{h}(u)+R_{m+1 / 2}(u)\right)=1 \text { a.e. (w.r.t. } \mu_{m+1}\right) u \in H^{m+\frac{1}{2}-\epsilon}
$$

and hence by the Egoroff Theorem we get

$$
\begin{gathered}
\forall \delta>0 \exists \Omega_{\delta} \subset H^{m+1 / 2-\epsilon}, \tilde{R}>0 \text { s.t. } \\
\mu_{m+1}\left(\Omega_{\delta}\right)>1-\delta \text { and } \prod_{j=0}^{2 m} \chi_{R}\left(E_{j / 2}(u)\right) \chi_{R}\left(\bar{h}(u)+R_{m+1 / 2}(u)\right)>1-\delta \\
\text { a.e. (w.r.t. } \left.\mu_{m+1}\right) u \in \Omega_{\delta} \text { and } \forall R>\tilde{R} .
\end{gathered}
$$

By combining this fact with (6.32) we deduce

$$
\mu_{m+1}\left\{\bigcup_{R>0} \operatorname{supp}\left(\prod_{j=0}^{2 m} \chi_{R}\left(E_{j / 2}(u)\right) \chi_{R}\left(\bar{h}(u)+R_{m+1 / 2}(u)\right) e^{-\bar{f}(u)}\right)\right\}=1 .
$$

Proof of Theorem 1.1 for $k=2(m+1)$. - It follows by combining Propositions 6.1, 6.5, 6.11. 


\section{Proof of Theorem 1.1 for $k=2 m+1, m>0$}

In this section we briefly describe how to adapt the proof of Theorem 1.1 given for $k=2(m+1)$ to the case $k=2 m+1$. We do not give the details of the proofs, however we underline the points where they have to be modified compared to the case $k=2(m+1)$. The following is an adapted version of Proposition 3.1.

Proposition 7.1. - Let $k \geq 2$ be a fixed integer. Then for every $R_{1}, R_{2}>0$ there is $C=C\left(R_{1}, R_{2}\right)>0$ such that

$$
\begin{aligned}
& \bigcap_{j=0}^{2 k-1}\left\{u \in H^{k-1 / 2}|| E_{j / 2}(u) \mid<R_{1}\right\} \cap\left\{u \in H^{k-1 / 2}|| E_{k}\left(\pi_{N} u\right)-\alpha_{N} \mid<R_{2}\right\} \\
& \subset\left\{u \in H^{k-\frac{1}{2}} \mid\|u\|_{H^{k-1 / 2}}<C\right\} \cap\left\{u \in H^{k-1 / 2}\left|\left\|\pi_{N} u\right\|_{\dot{H}^{k}}^{2}-\alpha_{N}\right|<C\right\}, \forall N \in \mathbb{N} .
\end{aligned}
$$

Proof. - It is similar to the proof of Proposition 3.1, hence we skip it.

By looking at (2.5), in analogy with our argument used to treat the conservation laws $E_{m+1}$, the most delicate terms to be treated in $E_{m+1 / 2}$ are of the type $\int p_{3}(u) d x$ where

$$
p_{3}(u) \in \mathscr{P}_{3}(u) \text { and } \tilde{p}_{3}(u)=u \partial_{x}^{m} u \partial_{x}^{m} u
$$

Next we present an adapted version of Propositions 5.1 and 5.2 in the case when $p_{3}(u)$ satisfies (7.2). We recall that the Sobolev spaces $H^{m-\epsilon}$ are a support for the measure $\mu_{m+1 / 2}$ for every $\epsilon>0$. This fact will be used in the sequel without any further comment.

Proposition 7.2. - Let $m \geq 1$ be a given integer and $p_{3}(u) \in \mathscr{P}_{3}(u)$ be such that

$$
\tilde{p}_{3}(u)=u \partial_{x}^{m} u \partial_{x}^{m} u \text {. }
$$

Then for every $\alpha \in\left(0, \frac{1}{2}\right)$ there exists $C=C(\alpha)>0$ such that

$$
\left\|f^{p_{3}}\left(\pi_{N} u\right)-f^{p_{3}}\left(\pi_{M} u\right)\right\|_{L^{p}\left(d \mu_{m+1 / 2}\right)} \leq C \frac{p^{3 / 2}}{(\min \{M, N\})^{\alpha}}, \forall M, N \in \mathbb{N}, p \geq 2
$$

where $f^{p_{3}}(v)=\int p_{3}(v) d x$. In particular

$$
\exists C>0 \text { s.t. } \mu_{m+1 / 2}\left(A_{M, N}^{p_{3}, \lambda}\right) \leq e^{-\frac{1}{C}\left(\lambda \min \{N, M\}^{\alpha}\right)^{2 / 3}}, \forall M, N \in \mathbb{N}, \lambda>0
$$

where

$$
A_{M, N}^{p_{3}, \lambda}=\left\{u \in H^{m-\epsilon}|| f^{p_{3}}\left(\pi_{N} u\right)-f^{p_{3}}\left(\pi_{M} u\right) \mid>\lambda\right\} .
$$

Proposition 7.3. - Let $m \geq 1$ be a given integer. There exists $C>0$ such that

$$
\left\|h_{N}\left(\pi_{N} u\right)-h_{M}\left(\pi_{M} u\right)\right\|_{L^{p}\left(d \mu_{m+1 / 2}\right)} \leq C \frac{p}{\sqrt{\min \{M, N\}}}, \forall M, N \in \mathbb{N}, p \geq 2
$$

where $h_{K}(v)=\|v\|_{\dot{H}^{m}}^{2}-\alpha_{K}$ for any $K \in \mathbb{N}$. In particular

$$
\exists C>0 \text { s.t. } \mu_{m+1 / 2}\left(B_{M, N}^{\lambda}\right) \leq e^{-\frac{1}{C}(\lambda \sqrt{\min \{N, M\}})}, \forall M, N \in \mathbb{N}, \lambda>0
$$

where

$$
B_{M, N}^{\lambda}=\left\{u \in H^{m-\epsilon}|| h_{N}\left(\pi_{N} u\right)-h_{M}\left(\pi_{N} u\right) \mid>\lambda\right\}
$$


Sketch of the proof. - The proof of Proposition 7.3 is identical to the proof of Proposition 5.2. Concerning the proof of Proposition 7.2 notice that (following the proof of Proposition 5.1) it is sufficient to prove (7.3) for $p=2$. By using the parametrization (1.3) (for $k=2 m+1$ ) we have to estimate

$$
\left\|\sum_{(i, j, k) \in \mathscr{G}_{M}^{N}} \frac{1}{\sqrt{|i|}|j|^{m+\frac{1}{2}} \sqrt{|k|}} \varphi_{i}(\omega) \varphi_{j}(\omega) \varphi_{k}(\omega)\right\|_{L_{\omega}^{2}}^{2}
$$

where $\mathscr{C}_{M}^{N}$ is the set defined in (5.34). By using Lemma 5.3 and arguing as in Lemma 5.4 we can estimate the quantity above by

$$
\sum_{(i, j) \in \mathbb{Z} \backslash\{0\}, i+j \neq 0,|i|>\frac{M}{2}} \frac{1}{|i||i+j|^{2 m+1}|j|} \leq \frac{C}{M^{\alpha}}
$$

for every $\alpha \in(0,1)$. The last estimate can be deduced by looking at the argument in [14] (see end of page 500).

Next we present a lemma allowing us to treat all the terms that appear in the expression of $E_{m+1 / 2}$ except the ones with the structure (7.2) (see (2.5)).

Lemma 7.4. - Let $m \geq 1$ be an integer and $p_{3}(u) \in \mathscr{P}_{3}(u)$ such that

$$
\tilde{p}_{3}(u)=\prod_{i=1}^{3} \partial_{x}^{\alpha_{i}} \text { with } \sum_{i=1}^{3} \alpha_{i}=2 m \text { and } 1 \leq \min _{i=1,2,3} \alpha_{i} \leq \max _{i=1,2,3} \alpha_{i} \leq m .
$$

Then for every $\epsilon>0, p \in[1, \infty)$ such that $\epsilon p>1$ there exists $C=C(\epsilon, p)>0$ such that:

$$
\left|\int p_{3}(u) d x\right| \leq C\|u\|_{H^{m-1 / 2}}^{2}\|u\|_{W^{m-1+\epsilon, p}} .
$$

Sketch of the proof. - We treat for simplicity the case $p_{3}(u)=\tilde{p}_{3}(u)$ (the general case can be treated by a similar argument). Next we also assume $\alpha_{1} \geq \alpha_{2} \geq \alpha_{3}$. Notice that by an integration by parts argument we can always reduce to the following two cases.

First case: $\alpha_{1}=m, \alpha_{2}=m-1, \alpha_{3}=1$.

In this case combine Lemma 3.3 with the Sobolev embedding $W^{\epsilon, p} \subset L^{\infty}$.

Second case: $\alpha_{1} \leq m-1$.

In this case we combine the Cauchy-Schwartz inequality with the Sobolev embedding $W^{\epsilon, p} \subset L^{\infty}$ and we get

$$
\left|\int p_{3}(u) d x\right| \leq\|u\|_{H^{m-1}}^{2}\left\|\partial_{x}^{m-1} u\right\|_{W^{\epsilon, p}}
$$

Lemma 7.5. - Let $m \geq 1$ be an integer and $p_{4}(u) \in \mathscr{P}_{4}(u)$ such that

$$
\tilde{p}_{4}(u)=u^{2} \partial_{x}^{m} u \partial_{x}^{m-1} u .
$$

Then for every $\epsilon>0, p \in[1, \infty)$ such that $\epsilon p>1$ there exists $C=C(\epsilon, p)>0$ such that:

$$
\left|\int p_{4}(u) d x\right| \leq C\|u\|_{H^{m-1 / 2}}\left(\|u\|_{H^{1}}^{2}\|u\|_{W^{m-1+\epsilon, p}}+\|u\|_{H^{m-1 / 2}}\|u\|_{H^{1}}^{2}\right) .
$$


Proof. - We treat for simplicity the case $p_{4}(u)=u^{2} \partial_{x}^{m} u \partial_{x}^{m-1} u$. Indeed in this specific case we could get the estimate as a direct application Lemma 3.3. However we propose a different and more robust proof that can be generalized for any $p_{4}(u)$ as in the assumptions. We start by the following inequality:

$$
\left|\int\left(\partial_{x} v_{1}\right) v_{2} d x\right| \leq\left\|v_{1}\right\|_{H^{1 / 2}}\left\|v_{2}\right\|_{H^{1 / 2}} .
$$

By using the above estimate, where we choose $v_{1}=\partial_{x}^{m-1} u$ and $v_{2}=u^{2} \partial_{x}^{m-1} u$, in conjunction with the following one:

$$
\left\|w_{1} w_{2}\right\|_{H^{1 / 2}} \leq C\left(\left\|w_{1}\right\|_{H^{1 / 2}}\left\|w_{2}\right\|_{L^{\infty}}+\left\|w_{1}\right\|_{L^{\infty}}\left\|w_{2}\right\|_{H^{1 / 2}}\right)
$$

we get

$$
\begin{aligned}
\left|\int p_{4}(u) d x\right| \leq C\left\|\partial_{x}^{m-1} u\right\|_{H^{1 / 2}}\left\|u^{2} \partial_{x}^{m-1} u\right\|_{H^{1 / 2}} \\
\quad \leq C\|u\|_{H^{m-1 / 2}}\left(\left\|u \partial_{x}^{m-1}\right\|_{H^{1 / 2}}\|u\|_{L^{\infty}}+\left\|u \partial_{x}^{m-1} u\right\|_{L^{\infty}}\|u\|_{H^{1 / 2}}\right) .
\end{aligned}
$$

By using again (7.12) in conjunction with the Sobolev embedding $W^{\epsilon, p} \subset L^{\infty}$ we get the result.

Lemma 7.6. - Let $m \geq 3$ be an integer and $p_{j}(u) \in \mathscr{P}_{j}(u)$ such that

$$
\tilde{p}_{j}(u)=\prod_{i=1}^{j} \partial_{x}^{\alpha_{i}} u \text { with } \sum_{i=1}^{j} \alpha_{i} \leq 2 m-1 \text { and } \max _{i=1, \ldots, j} \alpha_{i} \leq m-1 .
$$

Then there exists $C>0$ such that:

$$
\left|\int p_{j}(u) d x\right| \leq C\|u\|_{H^{m-1}}^{j} .
$$

Sketch of the proof. - We suppose $p_{j}(u)=\prod_{i=1}^{j} \partial_{x}^{\alpha_{i}} u$ with $\alpha_{1} \geq \cdots \geq \alpha_{j}$ (the general case works with a similar argument). By integration by parts we can reduce to two cases.

First case: $\alpha_{1}=m-1, \alpha_{2}=m-1$.

In this case by assumption we get $\alpha_{i} \leq 1$ for every $i=3, \ldots, j$. Hence by using the Cauchy-Schwartz inequality and the Sobolev embedding $H^{1} \subset L^{\infty}$ we get

$$
\left|\int p_{j}(u) d x\right| \leq\left\|\partial_{x}^{m-1} u\right\|_{L^{2}}^{2} \prod_{i=3}^{j}\|u\|_{H^{2}}
$$

Second case: $\alpha_{i} \leq m-2 \forall i=1, \ldots, j$.

By using the Sobolev embedding $H^{1} \subset L^{\infty}$ we get

$$
\left|\int p_{j}(u) d x\right| \leq \prod_{i=1}^{j}\left\|\partial_{x}^{\alpha_{i}} u\right\|_{H^{1}}
$$

and hence we conclude.

Next we give an adapted version of Lemma 6.6. Recall that the functions $h_{N}(u)$ and $f^{p_{3}}(u)$ are the ones introduced in Propositions 7.2 and 7.3. 
Lemma 7.7. - Let $m \geq 1$ be an integer and $p_{3}(u) \in \mathscr{P}_{3}(u)$ be such that

$$
\tilde{p}_{3}(u)=u \partial_{x}^{m} u \partial_{x}^{m} u \text {. }
$$

For every $R>0$ there exists $C=C(R)>0$ such that

$$
\mu_{m+1 / 2}\left\{u \in H^{m-\epsilon}|| f^{p_{3}}\left(\pi_{N} u\right)|>\lambda,| h_{N}\left(\pi_{N} u\right) \mid<R\right\} \leq C e^{-\frac{\lambda^{2}}{C \alpha_{N}^{2}}} \forall N \in \mathbb{N}, \lambda>0
$$

Sketch of the proof. - We have the following inequality

$$
\left|\int p_{3}\left(\pi_{N} u\right) d x\right| \leq C\left\|\pi_{N} u\right\|_{W^{\epsilon, p}}\left\|\pi_{N} u\right\|_{H^{m}}^{2} \leq C\left(\alpha_{N}+R\right)\left\|\pi_{N} u\right\|_{W^{\epsilon, p}}
$$

provided that $u$ belongs to the region on 1.h.s. of (7.14). The proof can be concluded by using the following estimate

$$
\mu_{m+1 / 2}\left\{u \in H^{m-\epsilon} \mid\|u\|_{W^{\epsilon, p}}>\lambda\right\} \leq C e^{-\frac{\lambda^{2}}{C}}, \forall \lambda>0
$$

whose proof is similar to the proof of Proposition 4.2 (the unique difference is to use along the proof the random vector $\varphi(\omega)=\sum_{n \neq 0} \frac{\varphi_{n}(\omega)}{|n|^{m+1 / 2}} e^{\mathbf{i} n x}$ instead of $\left.\varphi(\omega)=\sum_{n \neq 0} \frac{\varphi_{n}(\omega)}{|n|^{m+1}} e^{\mathbf{i} n x}\right)$.

The following version of Proposition 6.1 can be easily proved. Hence we skip its proof.

Proposition 7.8. - Let $m \geq 1$ and $\psi \in C_{c}(\mathbb{R})$ be given. Then there exist two functions $\bar{h}(u), \bar{f}(u)$ measurable with respect to $\mu_{m+1 / 2}$ such that:

$$
\begin{gathered}
|\bar{h}(u)|,|\bar{f}(u)|<\infty, \text { a.e. } u \in H^{m-\epsilon} \\
\prod_{j=0}^{2 m-1} \psi\left(E_{j / 2}\left(\pi_{N} u\right)\right) \psi\left(E_{m}\left(\pi_{N} u\right)-\alpha_{N}\right) e^{-R_{m+1 / 2}\left(\pi_{N} u\right)}
\end{gathered}
$$

converges in measure to

$$
\prod_{j=0}^{2 m-1} \psi\left(E_{j / 2}(u)\right) \psi\left(\bar{h}(u)+R_{m}(u)\right) e^{-\bar{f}(u)}
$$

Moreover

$$
\left.\left|E_{j / 2}(u)\right|,\left|R_{m}(u)\right|<\infty \text {, a.e. (w.r.t. } \mu_{m+1 / 2}\right) u \in H^{m-\epsilon} \forall j=0, \ldots, 2 m-1 .
$$

The proof of Proposition 6.11 can be easily adapted to give the following result.

Proposition 7.9. - Let $\bar{f}(u), \bar{h}(u)$ be as in Proposition 7.8 and $\chi_{R}$ as in Theorem 1.1. Then

$$
\left.\bigcup_{R>0} \operatorname{supp}\left(\prod_{j=0}^{2 m-1} \chi_{R}\left(E_{j / 2}(u)\right) \chi_{R}(\bar{h}(u))+R_{m}(u)\right) e^{-\bar{f}(u)}\right)=\operatorname{supp}\left(\mu_{m+1 / 2}\right) .
$$

The last step we need in order to prove Theorem 1.1 in the case $k=2 m+1$ is the following version of Proposition 6.5.

Proposition 7.10. - Let $m \geq 1$ and $\psi \in C_{c}(\mathbb{R})$ be given. For every $q \in[1, \infty)$ we have

$$
\sup _{N}\left\|\prod_{j=0}^{2 m-1} \psi\left(E_{j / 2}\left(\pi_{N} u\right)\right) \psi\left(E_{m}\left(\pi_{N} u\right)-\alpha_{N}\right) e^{-R_{m+1 / 2}\left(\pi_{N} u\right)}\right\|_{L^{q}\left(d \mu_{m+1 / 2}\right)}<\infty
$$


Sketch of the proof. - First case: $m \geq 2$.

Arguing as in the proof of Proposition 6.5 (as in the case $m>0$ ) and by using Lemma 7.7, Proposition 7.2 and 7.3 we can prove (7.15) provided that $R_{m+1 / 2}$ is replaced by $f^{p_{3}}$ with $p_{3}(u)$ that satisfy (7.2).

Second case: $m=1$.

Arguing as in the proof of Proposition 6.5 (as in the case $m=0$ ) and by using an adapted version of Lemma 7.7 (in the same spirit as Lemma 6.10) we can prove (7.15) provided that $R_{1+1 / 2}$ is replaced by $f^{p_{3}}$ with $p_{3}(u)$ that satisfy (7.2) with $m=1$.

Hence the proof of (7.15) follows provided that we prove the following claim (see the analogous claim stated along the proof of Proposition 6.5).

ClaIm. - It is sufficient to prove (7.15) with $R_{m+1 / 2}\left(\pi_{N} u\right)$ replaced by $f^{p_{3}}\left(\pi_{N} u\right)$ where

$$
\tilde{p}_{3}(u)=u \partial_{x}^{m} u \partial_{x}^{m} u \text {. }
$$

Due to Proposition 7.1 and due to the cut-off function $\psi$ we deduce that the $L^{q}$ norms (that appear in (7.15)) are actually computed in the region $\Omega_{N}$ given by the condition

$$
\Omega_{N}=\left\{u \in H^{m-\epsilon} \mid\left\|\pi_{N} u\right\|_{H^{m-1 / 2}}<C\right\}
$$

where $C>0$ is independent on $N$.

Next we prove the claim.

First case: $m \geq 3$.

By looking at (2.5) it is sufficient to prove that

$$
\sup _{N}\left\|e^{q\left|f^{p_{j}}\left(\pi_{N} u\right)\right|}\right\|_{L^{1}\left(\Omega_{N}, d \mu_{m+1 / 2}\right)}<\infty
$$

where $p_{j}(u)$ satisfy (7.9), (7.10) and (7.13). Notice that if $p_{j}(u)$ satisfies (7.13) then in the region $\Omega_{N}$ (see (7.17)) we get $\sup _{N}\left\|f^{p_{j}}\left(\pi_{N} u\right)\right\|_{L^{\infty}\left(\Omega_{N}\right)}<\infty$ (where we have used Lemma 7.6) and hence we deduce (7.18). Next we treat the case when $p_{3}(u)$ satisfies (7.9). In this case by Lemma 7.4 we get $\left|f^{p_{3}}\left(\pi_{N} u\right)\right| \leq C\left\|\pi_{N} u\right\|_{W^{m-1+\epsilon, p}}$, provided that $u \in \Omega_{N}$. In particular

$$
\left\{u \in \Omega_{N} \| f^{p_{3}}\left(\pi_{N} u\right) \mid>\lambda\right\} \subset\left\{u \in \Omega_{N} \mid\left\|\pi_{N} u\right\|_{W^{m-1+\epsilon, p}}>\lambda C^{-1}\right\}
$$

and hence (by using a suitable version of Proposition 4.2)

$$
\left.\mu_{m+1 / 2}\left\{u \in \Omega_{N}|| f^{p_{3}}\left(\pi_{N} u\right) \mid>\lambda\right\}\right) \leq C e^{-\frac{\lambda^{2}}{C}}
$$

for a suitable $C>0$. As a consequence we get

$$
\begin{gathered}
\sup _{N} \int_{\Omega_{N}} e^{q \mid f^{p_{3}\left(\pi_{N} u\right) \mid} d \mu_{m+1 / 2}} \\
=\sup _{N} \int_{0}^{\infty} e^{q \lambda} d \mu_{m+1 / 2}\left\{u \in \Omega_{N}|| f^{p_{3}}\left(\pi_{N} u\right) \mid>\lambda\right\} d \lambda<\infty .
\end{gathered}
$$

With a similar argument we can prove $\sup _{N}\left\|e^{q\left|f^{p}\left(\pi_{N} u\right)\right|}\right\|_{L^{1}\left(\Omega_{N}, d \mu_{m+1 / 2}\right)}<\infty$ with $p(u)$ as in (7.10).

Second case: $m=1$.

Looking at the structure of $E_{3 / 2}$ (see the introduction) we have to show that $\sup _{N}\left\|e^{q\left|f^{p}\left(\pi_{N} u\right)\right|}\right\|_{L^{1}\left(\Omega_{N}, d \mu_{3 / 2}\right)}<\infty$ where $p(u)=u^{3} H \partial_{x} u, p(u)=u^{2} H\left(u \partial_{x} u\right), p(u)=u^{5}$. 
Notice that by the Sobolev embedding $H^{1 / 2} \subset L^{5}$ we get

$$
\left|\int\left(\pi_{N} u\right)^{5} d x\right| \leq C\left\|\pi_{N} u\right\|_{H^{1 / 2}}^{5}<C, \forall u \in \Omega_{N}
$$

(see (7.17) for $m=1$ ) and hence we get the desired bound when $p(u)=u^{5}$.

Next we treat the term $p(u)=u^{3} H \partial_{x} u$ (the term $p(u)=u^{2} H\left(u \partial_{x} u\right)$ can be treated in a similar way). By using (7.11) (used along the proof of Lemma 7.5) in conjunction with the estimate

$$
\left\|v_{1} v_{2}\right\|_{H^{1 / 2}} \leq C\left(\left\|v_{1}\right\|_{H^{1 / 2}}\left\|v_{2}\right\|_{L^{\infty}}+\left\|v_{2}\right\|_{H^{1 / 2}}\left\|v_{1}\right\|_{L^{\infty}}\right)
$$

we get

$$
\left|\int\left(\pi_{N} u\right)^{3}\left(H \partial_{x} \pi_{N} u\right) d x\right| \leq C\left\|\pi_{N} u\right\|_{H^{1 / 2}}^{2}\left\|\pi_{N} u\right\|_{L^{\infty}}^{2} \leq C\left\|\pi_{N} u\right\|_{L^{\infty}}^{2}, \forall u \in \Omega_{N}
$$

and hence by Sobolev embedding $H^{\frac{2}{3}} \subset L^{\infty}$ we get

$$
\cdots \leq C\left\|\pi_{N} u\right\|_{H^{2 / 3}}^{2} \leq C\left\|\pi_{N} u\right\|_{H^{1 / 2}}\left\|\pi_{N} u\right\|_{H^{5 / 6}}, \forall u \in \Omega_{N} .
$$

Then we deduce

$$
\left\{u \in \Omega_{N}|| f^{p}\left(\pi_{N} u\right) \mid>\lambda\right\} \subset\left\{u \in \Omega_{N} \mid\left\|\pi_{N} u\right\|_{H^{5 / 6}}>\lambda C^{-1}\right\}
$$

and hence (by using a suitable version of Proposition 4.2)

$$
\mu_{3 / 2}\left\{u \in \Omega_{N}|| f^{p}\left(\pi_{N} u\right) \mid>\lambda\right\} \leq C e^{-\frac{\lambda^{2}}{C}} .
$$

In particular

$$
\sup _{N}\left\|e^{q\left|f^{p}\left(\pi_{N} u\right)\right|}\right\|_{L^{1}\left(\Omega_{N}, d \mu_{3 / 2}\right)} \leq \sup _{N} \int_{0}^{\infty} e^{q \lambda} e^{-\frac{\lambda^{2}}{C}} d \lambda<\infty
$$

Third case: $m=2$.

The bound $\sup _{N}\left\|e^{q\left|f^{p}\left(\pi_{N} u\right)\right|}\right\|_{L^{1}\left(\Omega_{N}, d \mu_{5 / 2}\right)}<\infty$ follows by Lemma 7.4 in conjunction with a suitable version of Proposition 4.2 (used in the same spirit as above), in the case $p(u) \in \mathscr{P}_{3}(u)$ (but $p(u)$ does not satisfy (7.16)). If $p(u) \in \mathscr{P}_{4}(u)$ satisfies (7.10) then we can conclude by using Lemma 7.5 in conjunction with a suitable version of Proposition 4.2.

Next we treat the case

$$
p_{4}(u) \in \mathscr{P}_{4}(u) \text { such that } \tilde{p}_{4}(u)=u\left(\partial_{x} u\right)^{3} .
$$

By combining the Hölder inequality with the Sobolev embedding $W^{\epsilon, p} \subset L^{\infty}$ (provided that $\epsilon p>1$ ) we get

$$
\begin{gathered}
\left|\int p_{4}\left(\pi_{N} u\right) d x\right| \leq C\left\|\pi_{N} u\right\|_{H^{1}}^{2}\left\|\partial_{x} \pi_{N} u\right\|_{L^{\infty}}\left\|\pi_{N} u\right\|_{L^{\infty}} \\
\leq C\left\|\pi_{N} u\right\|_{H^{1}}^{3}\left\|\partial_{x} \pi_{N} u\right\|_{W^{\epsilon, p}} \leq C\left\|\pi_{N} u\right\|_{W^{1+\epsilon, p}}, \forall u \in \Omega_{N} .
\end{gathered}
$$

Hence we can conclude as in the previous cases by using a suitable version of Proposition 4.2.

Finally notice that by using the Sobolev embedding $H^{1} \subset L^{\infty}$ for any $p \in[1, \infty)$ we get $\sup _{N} e^{q \mid f^{p}\left(\pi_{N}(u) \mid\right.}<\infty$ in the cases $p(u) \in \mathscr{P}_{5}(u)$ and $\tilde{p}(u)=\left(\partial_{x} u\right)^{2} u^{3}, p(u) \in \mathscr{P}_{6}(u)$ and $\tilde{p}(u)=u^{5} \partial_{x} u, p(u)=u^{7}$.

The proof of the claim is concluded. 


\section{Computation of $\frac{d}{d t} E_{m+1}\left(\pi_{N} u(t, x)\right)$}

In this section we shall use the notations introduced in Section 2. Our aim is to construct for every $N \in \mathbb{N}$ and for every fixed $m \in \mathbb{N}$ a function

$$
G_{m+1, N}: \mathcal{T}_{N} \rightarrow \mathbb{R}
$$

where

$$
\mathcal{J}_{N}=\left\{\sum_{|j| \in(0, N]} c_{j} e^{\mathbf{i} j x} \mid \bar{c}_{j}=c_{-j}\right\}
$$

and such that

$$
\frac{d}{d t} E_{m+1}\left(\pi_{N} u(t, x)\right)=G_{m+1, N}\left(\pi_{N} u(t, x)\right)
$$

where $u(t, x)$ are solutions to the truncated Benjamin-Ono Equation (1.6).

First we introduce some preliminary notations.

To $p(u) \in \cup_{n=2}^{\infty} \mathscr{P}_{n}(u)$ we associate a new object dependent on $N \in \mathbb{N}$ that will be denoted by $p_{N}^{*}(u)$.

Let $p(u)$ be such that

$$
\tilde{p}(u)=\prod_{i=1}^{n} \partial_{x}^{\alpha_{i}} u
$$

for suitable $0 \leq \alpha_{1} \leq \cdots \leq \alpha_{n}$ and $\alpha_{i} \in \mathbb{N}$. First we define $p_{i, N}^{*}(u)$ as the function obtained by $p(u)$ replacing $\partial_{x}^{\alpha_{i}}(u)$ by $\partial_{x}^{\alpha_{i}}\left(\pi_{>N}\left(u \partial_{x} u\right)\right)$, i.e.,

$$
p_{i, N}^{*}(u)=p(u)_{\mid \partial_{x}^{\alpha_{i}} u=\partial_{x}^{\alpha_{i}}\left(\pi_{>N}\left(u \partial_{x} u\right)\right)}, \forall i=1, . ., n
$$

where

$$
\pi_{>N}\left(\sum c_{j} e^{\mathbf{i} j x}\right)=\sum_{|j|>N} c_{j} e^{\mathbf{i} j x}
$$

We now define $p_{N}^{*}(u)$ as follows:

$$
p_{N}^{*}(u)=\sum_{i=1}^{n} p_{i, N}^{*}(u) .
$$

EXAmple 8.1. - In order to clarify the definition of $p_{N}^{*}(u)$ we give an example.

Assume

$$
p(u)=\partial_{x}^{\alpha} u H\left(\partial_{x}^{\beta} u\left(H \partial_{x}^{\gamma} u\right)\right)
$$

then

$$
\begin{aligned}
p_{N}^{*}(u)= & \partial_{x}^{\alpha}\left(\pi_{>N}\left(u \partial_{x} u\right)\right) H\left(\partial_{x}^{\beta} u\left(H \partial_{x}^{\gamma} u\right)\right) \\
& +\partial_{x}^{\alpha} u H\left(\partial_{x}^{\beta}\left(\pi_{>N}\left(u \partial_{x} u\right)\right)\left(H \partial_{x}^{\gamma} u\right)\right)+\partial_{x}^{\alpha} u H\left(\partial_{x}^{\beta} u\left(H \partial_{x}^{\gamma}\left(\pi_{>N}\left(u \partial_{x} u\right)\right)\right)\right)
\end{aligned}
$$

REMARK 8.2. - Notice that if $p(u) \in \mathscr{P}_{n}(u)$ (i.e., $p(u)$ is homogeneous of order $n$ w.r.t. $u$ ) then $p_{N}^{*}(u)$ is a function homogeneous of order $n+1$ for every $N \in \mathbb{N}$.

We are now able to describe the function $G_{m+1, N}$ (see (1.7)). 
Proposition 8.3. - For every fixed integer $m \geq 0$ and for every $N \in \mathbb{N}$ we have:

$$
\begin{aligned}
\frac{d}{d t} E_{m+1}\left(\pi_{N} u(t)\right)= & \sum_{\substack{p(u) \in \mathscr{P}_{3}(u) s . t . \\
\tilde{p}(u)=u \partial_{x}^{m} u \partial_{x}^{m+1} u}} c_{2(m+1)}(p) \int p_{N}^{*}\left(\pi_{N} u(t)\right) d x \\
& +\sum_{\substack{p(u) \in \mathscr{P}_{j}(u) s . t . j=3, \ldots, 2 m+4 \\
\|p(u)\|=2 m-j+4 \\
|p(u)| \leq m}} c_{2(m+1)}(p) \int p_{N}^{*}\left(\pi_{N} u(t)\right) d x \\
&
\end{aligned}
$$

where $u(t, x)$ solves (1.6) and $c_{2(m+1)}(p)$ are the same constants that appear in (2.4) for $k=2(m+1)$.

Sketch of the proof. - We follow [15] (Lemma IV.3.5 page 127).

Let $\left.p(u) \in \mathscr{P}_{h}(u)\right)$ be such that $\tilde{p}(u)=\prod_{i=1}^{h} \partial_{x}^{\alpha_{i}} u$. Then by elementary calculus

$$
\frac{d}{d t} \int p(u(t, x)) d x=\sum_{i=1}^{h} \int p(u)_{\mid \partial_{x}^{\alpha_{i}} u=\partial_{x}^{\alpha_{i}} \partial_{t} u} d x
$$

where $u(t, x)$ is any regular time-dependent function. Motivated by the identity above we introduce

$$
p_{t}(u)=\sum_{i=1}^{h} p(u)_{\mid \partial_{x}^{\alpha_{i}} u=\partial_{x}^{\alpha_{i}} \partial_{t} u} .
$$

By looking at the structure of $E_{m+1}$ (see (2.4)) we get

$$
\begin{aligned}
\frac{d}{d t} E_{m+1} u(t, x)= & 2 \int \partial_{x}^{m+1} u \partial_{x}^{m+1} \partial_{t} u d x+\sum_{\substack{p(u) \in \mathscr{P}_{3}(u) s . t . \\
\tilde{p}(u)=u \partial_{x}^{m-1} u \partial_{x}^{m} u}} c_{2(m+1)}(p) \int p_{t}(u) d x \\
& +\sum_{\substack{p(u) \in \mathscr{P}_{j}(u) s . t . j=3, \ldots, 2 m+4 \\
\|p(u)\|=2 m-j+4 \\
|p(u)| \leq m}} c_{2(m+1)}(p) \int p_{t}(u) d x \\
&
\end{aligned}
$$

where $u(t, x)$ is any given time dependent function.

Next notice that if $u(t, x)$ solves (1.6) then (due to the properties $\pi_{N}^{2}=\pi_{N}$ and $\left.\pi_{N}+\pi_{>N}=\mathrm{Id}\right)$

$$
\partial_{t} \pi_{N} u+H \partial_{x}^{2} \pi_{N} u+\left(\left(\pi_{N} u\right) \partial_{x}\left(\pi_{N} u\right)\right)=\pi_{>N}\left(\left(\pi_{N} u\right) \partial_{x}\left(\pi_{N} u\right)\right)
$$

and hence if we choose in (8.4) $u(t, x)=\pi_{N} u(t, x)$ then we can replace the derivative $\partial_{t} \pi_{N} u(t, x)$, that appears on the r.h.s. of (8.4), by the expression

$$
-H \partial_{x}^{2} \pi_{N} u-\left(\left(\pi_{N} u\right)\left(\partial_{x} \pi_{N} u\right)\right)+\pi_{>N}\left(\left(\pi_{N} u\right)\left(\partial_{x} \pi_{N} u\right)\right) .
$$

Notice that if we replace $\partial_{t}\left(\pi_{N} u\right)$ by the term $-H \partial_{x}^{2} \pi_{N} u-\left(\left(\pi_{N} u\right)\left(\partial_{x} \pi_{N} u\right)\right)$ then we get zero on the r.h.s. of (8.4) (in fact in this way we are dealing with $\pi_{N} u(t, x)$ as with an exact solution of the Benjamin-Ono equation). However the contribution that we get when we replace $\partial_{t}\left(\pi_{N} u\right)$ by the term $\pi_{>N}\left(\left(\pi_{N} u\right) \partial_{x}\left(\pi_{N} u\right)\right)$ is not trivial (in fact looking at (8.5) this term reflects how far is $\pi_{N} u(t, x)$ from being a precise solution of the Benjamin-Ono equation). 
Hence we deduce (8.3) once we notice that in the construction above there is no contribution coming from the quadratic part of $E_{m+1}$. In fact this contribution is given by the following quantity

$$
\int \partial_{x}^{m+1}\left(\pi_{N} u\right) \partial_{x}^{m+1} \pi_{>N}\left(\left(\pi_{N} u\right) \partial_{x}\left(\pi_{N} u\right)\right) d x
$$

which is zero by orthogonality ( $\pi_{N} u$ is localized on the $n$ modes with $|n| \leq N$ and $\pi_{>N}\left(\left(\pi_{N} u\right) \partial_{x}\left(\pi_{N} u\right)\right)$ is localized in the complementary modes).

\section{Some algebraic identities}

The results of this section will be useful along the proof of Theorem 1.2. We recall the notation $\pi_{>N}=\operatorname{Id}-\pi_{N}$. Moreover given a function $u(x)$ we define

$$
u^{+}=\pi_{+} u \text { and } u^{-}=\pi_{-} u
$$

where $\pi_{+}$(resp. $\pi_{-}$) is the projector on the positive (resp. the negative) frequencies. We recall also that

$$
H\left(\sum_{j \in \mathbb{Z} \backslash\{0\}} c_{j} e^{\mathbf{i} j x}\right)=-\mathbf{i} \sum_{j>0} c_{j} e^{\mathbf{i} j x}+\mathbf{i} \sum_{j<0} c_{j} e^{\mathbf{i} j x}
$$

and $\mathcal{J}_{N}$ is defined by (8.1).

Lemma 9.1. - Let $u \in \mathcal{T}_{N}$ be such that $\int u d x=0$. Then the following identities occur:

$$
\begin{aligned}
\int u\left(H \partial_{x}^{m} \pi_{>N}\left(u \partial_{x} u\right)\right) \partial_{x}^{m+1} u d x= & \sum_{j=1}^{m} a_{j}\left[\int \pi_{>N}\left(\partial_{x}^{j} u^{+} \partial_{x}^{m-j+1} u^{+}\right) \pi_{>N}\left(u^{-} \partial_{x}^{m+1} u^{-}\right)\right. \\
& \left.-\pi_{>N}\left(\partial_{x}^{j} u^{-} \partial_{x}^{m-j+1} u^{-}\right) \pi_{>N}\left(u^{+} \partial_{x}^{m+1} u^{+}\right)\right]
\end{aligned}
$$

for suitable coefficient $a_{j} \in \mathbb{C}$;

$$
\begin{aligned}
\int u\left(H \partial_{x}^{m} u\right) \partial_{x}^{m+1} \pi_{>N}\left(u \partial_{x} u\right) d x= & \sum_{j=1}^{m} b_{j}\left[\int \pi_{>N}\left(\partial_{x}^{j} u^{+} \partial_{x}^{m-j+1} u^{+}\right) \pi_{>N}\left(u^{-} \partial_{x}^{m+1} u^{-}\right)\right. \\
& \left.-\pi_{>N}\left(\partial_{x}^{j} u^{-} \partial_{x}^{m-j+1} u^{-}\right) \pi_{>N}\left(u^{+} \partial_{x}^{m+1} u^{+}\right)\right]
\end{aligned}
$$

for suitable coefficient $b_{j} \in \mathbb{C}$.

REMARK 9.2. - Notice that the 1.h.s. of (9.1) and (9.2) involve at first insight (after developing the $m$-derivative of the product) a term that contains the product of two derivatives of order $m+1$, which is quite dangerous (see the end of Section 1.3). The main point of the lemma above is that on the r.h.s. of (9.1) and (9.2) this bad term is disappeared.

Proof. - We prove (9.1). Due to the following identity

$$
\int\left(\pi_{>N} f\right) g d x=\int\left(\pi_{>N} f\right)\left(\pi_{>N} g\right) d x
$$

we get:

$$
\int u\left(H \partial_{x}^{m} \pi_{>N}\left(u \partial_{x} u\right)\right) \partial_{x}^{m+1} u d x=\int\left(H \partial_{x}^{m} \pi_{>N}\left(u \partial_{x} u\right)\right) \pi_{>N}\left(u \partial_{x}^{m+1} u\right) d x .
$$

4 e SÉRIE - TOME $46-2013$ - No 2 
On the other hand if $v(x), w(x)$ are trigonometric polynomials of degree $N$ we have

$$
\pi_{>N}\left(v^{+} w^{-}\right)=0
$$

and in particular

$$
\pi_{>N}(v w)=\pi_{>N}\left(v^{+} w^{+}\right)+\pi_{>N}\left(v^{-} w^{-}\right) .
$$

As a consequence we continue the identity above as follows

$$
\begin{aligned}
\cdots= & -\mathbf{i} \int \partial_{x}^{m} \pi_{>N}\left(u^{+} \partial_{x} u^{+}\right) \pi_{>N}\left(u^{-} \partial_{x}^{m+1} u^{-}\right) d x \\
& +\mathbf{i} \int \partial_{x}^{m} \pi_{>N}\left(u^{-} \partial_{x} u^{-}\right) \pi_{>N}\left(u^{+} \partial_{x}^{m+1} u^{+}\right) d x
\end{aligned}
$$

where we have used the definition of the Hilbert transform $H$,

$$
\begin{aligned}
\cdots= & -\mathbf{i} \int \pi_{>N}\left(u^{+} \partial_{x}^{m+1} u^{+}\right) \pi_{>N}\left(u^{-} \partial_{x}^{m+1} u^{-}\right)+\mathbf{i} \int \pi_{>N}\left(u^{-} \partial_{x}^{m+1} u^{-}\right) \pi_{>N}\left(u^{+} \partial_{x}^{m+1} u^{+}\right) d x \\
& -\mathbf{i} \int \pi_{>N}\left(\partial_{x}^{m}\left(u^{+} \partial_{x} u^{+}\right)-u^{+} \partial_{x}^{m+1} u^{+}\right) \pi_{>N}\left(u^{-} \partial_{x}^{m+1} u^{-}\right) d x \\
& +\mathbf{i} \int \pi_{>N}\left(\partial_{x}^{m}\left(u^{-} \partial_{x} u^{-}\right)-u^{-} \partial_{x}^{m+1} u^{-}\right) \pi_{>N}\left(u^{+} \partial_{x}^{m+1} u^{+}\right) d x .
\end{aligned}
$$

We can conclude by the Leibniz rule since the first two terms above cancel. Concerning (9.2) notice that by using (9.3) and (9.4) we get:

$$
\begin{aligned}
\int u\left(H \partial_{x}^{m} u\right) \partial_{x}^{m+1} \pi_{>N}\left(u \partial_{x} u\right) d x= & \mathbf{i} \int \partial_{x}^{m+1} \pi_{>N}\left(u^{+} \partial_{x} u^{+}\right) \pi_{>N}\left(u^{-} \partial_{x}^{m} u^{-}\right) d x \\
& -\mathbf{i} \int \partial_{x}^{m+1} \pi_{>N}\left(u^{-} \partial_{x} u^{-}\right) \pi_{>N}\left(u^{+} \partial_{x}^{m} u^{+}\right) d x
\end{aligned}
$$

and by integration by parts

$\cdots=-\mathbf{i} \int \partial_{x}^{m} \pi_{>N}\left(u^{+} \partial_{x} u^{+}\right) \pi_{>N} \partial_{x}\left(u^{-} \partial_{x}^{m} u^{-}\right)+\mathbf{i} \int \partial_{x}^{m} \pi_{>N}\left(u^{-} \partial_{x} u^{-}\right) \pi_{>N} \partial_{x}\left(u^{+} \partial_{x}^{m} u^{+}\right) d x$ which in turn gives

$$
\begin{aligned}
\cdots= & -\mathbf{i} \int \partial_{x}^{m} \pi_{>N}\left(u^{+} \partial_{x} u^{+}\right) \pi_{>N}\left(\partial_{x} u^{-} \partial_{x}^{m} u^{-}\right)+\mathbf{i} \int \partial_{x}^{m} \pi_{>N}\left(u^{-} \partial_{x} u^{-}\right) \pi_{>N}\left(\partial_{x} u^{+} \partial_{x}^{m} u^{+}\right) d x \\
& -\mathbf{i} \int \partial_{x}^{m} \pi_{>N}\left(u^{+} \partial_{x} u^{+}\right) \pi_{>N}\left(u^{-} \partial_{x}^{m+1} u^{-}\right)+\mathbf{i} \int \partial_{x}^{m} \pi_{>N}\left(u^{-} \partial_{x} u^{-}\right) \pi_{>N}\left(u^{+} \partial_{x}^{m+1} u^{+}\right) d x .
\end{aligned}
$$

Notice that the last two integrals above

$$
-\mathbf{i} \int \partial_{x}^{m} \pi_{>N}\left(u^{+} \partial_{x} u^{+}\right) \pi_{>N}\left(u^{-} \partial_{x}^{m+1} u^{-}\right)+\mathbf{i} \int \partial_{x}^{m} \pi_{>N}\left(u^{-} \partial_{x} u^{-}\right) \pi_{>N}\left(u^{+} \partial_{x}^{m+1} u^{+}\right) d x
$$

can be treated as in (9.5). Hence we have to deal with the remaining terms in the identity above:

$$
-\mathbf{i} \int \partial_{x}^{m} \pi_{>N}\left(u^{+} \partial_{x} u^{+}\right) \pi_{>N}\left(\partial_{x} u^{-} \partial_{x}^{m} u^{-}\right)+\mathbf{i} \int \partial_{x}^{m} \pi_{>N}\left(u^{-} \partial_{x} u^{-}\right) \pi_{>N}\left(\partial_{x} u^{+} \partial_{x}^{m} u^{+}\right) d x .
$$

Those integrals can be easily handled by using the Leibniz rule. 
In the same spirit as in Lemma 9.1 one can prove that if $u \in \mathcal{T}_{N}$ is such that $\int u d x=0$, then the following identities occur:

$$
\begin{aligned}
\int u\left(\partial_{x}^{m} \pi_{>N}\left(u \partial_{x} u\right)\right) \partial_{x}^{m+1}(H u) d x= & \sum_{j=1}^{m} c_{j}\left[\int \pi_{>N}\left(\partial_{x}^{j} u^{+} \partial_{x}^{m-j+1} u^{+}\right) \pi_{>N}\left(u^{-} \partial_{x}^{m+1} u^{-}\right)\right. \\
& \left.-\pi_{>N}\left(\partial_{x}^{j} u^{-} \partial_{x}^{m-j+1} u^{-}\right) \pi_{>N}\left(u^{+} \partial_{x}^{m+1} u^{+}\right)\right]
\end{aligned}
$$

for suitable $c_{j} \in \mathbb{C}$;

$$
\begin{aligned}
\int u \partial_{x}^{m} u\left(\partial_{x}^{m+1} H \pi_{>N}\left(u \partial_{x} u\right)\right) d x= & \sum_{j=1}^{m} d_{j}\left[\int \pi_{>N}\left(\partial_{x}^{j} u^{+} \partial_{x}^{m-j+1} u^{+}\right) \pi_{>N}\left(u^{-} \partial_{x}^{m+1} u^{-}\right)\right. \\
& \left.-\pi_{>N}\left(\partial_{x}^{j} u^{-} \partial_{x}^{m-j+1} u^{-}\right) \pi_{>N}\left(u^{+} \partial_{x}^{m+1} u^{+}\right)\right]
\end{aligned}
$$

for suitable $d_{j} \in \mathbb{C}$;

(9.8)

$$
\begin{aligned}
\int H u\left(\partial_{x}^{m} \pi_{>N} H\left(u \partial_{x} u\right)\right)\left(\partial_{x}^{m+1} H u\right) d x= & \sum_{j=1}^{m} e_{j}\left[\int \pi_{>N}\left(\partial_{x}^{j} u^{+} \partial_{x}^{m-j+1} u^{+}\right) \pi_{>N}\left(u^{-} \partial_{x}^{m+1} u^{-}\right)\right. \\
& \left.-\pi_{>N}\left(\partial_{x}^{j} u^{-} \partial_{x}^{m-j+1} u^{-}\right) \pi_{>N}\left(u^{+} \partial_{x}^{m+1} u^{+}\right)\right]
\end{aligned}
$$

for suitable $e_{j} \in \mathbb{C}$;

$$
\begin{aligned}
\int H u\left(\partial_{x}^{m} H u\right)\left(\partial_{x}^{m+1} \pi_{>N} H\left(u \partial_{x} u\right)\right) d x= & \sum_{j=1}^{m} f_{j}\left[\int \pi_{>N}\left(\partial_{x}^{j} u^{+} \partial_{x}^{m-j+1} u^{+}\right) \pi_{>N}\left(u^{-} \partial_{x}^{m+1} u^{-}\right)\right. \\
& \left.-\pi_{>N}\left(\partial_{x}^{j} u^{-} \partial_{x}^{m-j+1} u^{-}\right) \pi_{>N}\left(u^{+} \partial_{x}^{m+1} u^{+}\right)\right]
\end{aligned}
$$

for suitable $f_{j} \in \mathbb{C}$;

$$
\begin{aligned}
\int H u\left(\partial_{x}^{m} \pi_{>N}\left(u \partial_{x} u\right)\right)\left(\partial_{x}^{m+1} u\right) d x= & \sum_{j=1}^{m} g_{j}\left[\int \pi_{>N}\left(\partial_{x}^{j} u^{+} \partial_{x}^{m-j+1} u^{+}\right) \pi_{>N}\left(u^{-} \partial_{x}^{m+1} u^{-}\right)\right. \\
& \left.-\pi_{>N}\left(\partial_{x}^{j} u^{-} \partial_{x}^{m-j+1} u^{-}\right) \pi_{>N}\left(u^{+} \partial_{x}^{m+1} u^{+}\right)\right]
\end{aligned}
$$

for suitable $g_{j} \in \mathbb{C}$;

$$
\begin{aligned}
\int H u\left(\partial_{x}^{m} u\right)\left(\partial_{x}^{m+1} \pi_{>N}\left(u \partial_{x} u\right)\right) d x= & \sum_{j=1}^{m} h_{j}\left[\int \pi_{>N}\left(\partial_{x}^{j} u^{+} \partial_{x}^{m-j+1} u^{+}\right) \pi_{>N}\left(u^{-} \partial_{x}^{m+1} u^{-}\right)\right. \\
& \left.-\pi_{>N}\left(\partial_{x}^{j} u^{-} \partial_{x}^{m-j+1} u^{-}\right) \pi_{>N}\left(u^{+} \partial_{x}^{m+1} u^{+}\right)\right]
\end{aligned}
$$

for suitable $h_{j} \in \mathbb{C}$. 
Lemma 9.3. - Let $u \in \mathcal{T}_{N}$ be such that $\int u d x=0$. Then the following identities occur:

$$
\begin{aligned}
& \int u\left(H \partial_{x}^{m} \pi_{>N}\left(u \partial_{x} u\right)\right)\left(\partial_{x}^{m+1} H u\right) d x+\int u\left(H \partial_{x}^{m} u\right)\left(\partial_{x}^{m+1} \pi_{>N} H\left(u \partial_{x} u\right)\right) d x \\
= & -\int \partial_{x}^{m}\left(\pi_{>N}\left(u^{+} \partial_{x} u^{+}\right)\right) \pi_{>N}\left(\partial_{x} u^{-} \partial_{x}^{m} u^{-}\right) d x-\int \partial_{x}^{m}\left(\pi_{>N}\left(u^{-} \partial_{x} u^{-}\right)\right) \pi_{>N}\left(\partial_{x} u^{+} \partial_{x}^{m} u^{+}\right) d x .
\end{aligned}
$$

REMARK 9.4. - To understand the interest of Lemma 9.3, see Remark 9.2.

Proof. - By combining (9.3) with (9.4) we get:

$$
\begin{aligned}
& \int u\left(H \partial_{x}^{m} \pi_{>N}\left(u \partial_{x} u\right)\right)\left(\partial_{x}^{m+1} H u\right) d x+\int u\left(H \partial_{x}^{m} u\right)\left(\partial_{x}^{m+1} H\left(\pi_{>N}\left(u \partial_{x} u\right)\right)\right) d x \\
& =\int\left(\pi_{>N} \partial_{x}^{m}\left(u^{+} \partial_{x} u^{+}\right)\right) \pi_{>N}\left(u^{-} \partial_{x}^{m+1} u^{-}\right) d x+\int\left(\pi_{>N} \partial_{x}^{m}\left(u^{-} \partial_{x} u^{-}\right)\right) \pi_{>N}\left(u^{+} \partial_{x}^{m+1} u^{+}\right) d x \\
& \quad+\int \partial_{x}^{m+1}\left(\pi_{>N}\left(u^{+} \partial_{x} u^{+}\right)\right) \pi_{>N}\left(u^{-} \partial_{x}^{m} u^{-}\right) d x+\int\left(\pi_{>N} \partial_{x}^{m+1}\left(u^{-} \partial_{x} u^{-}\right)\right) \pi_{>N}\left(u^{+} \partial_{x}^{m} u^{+}\right) d x
\end{aligned}
$$

On the other hand by integration by parts in the second term we get:

$$
\begin{aligned}
& \int\left(\pi_{>N} \partial_{x}^{m}\left(u^{+} \partial_{x} u^{+}\right)\right) \pi_{>N}\left(u^{-} \partial_{x}^{m+1} u^{-}\right)+\int \partial_{x}^{m+1}\left(\pi_{>N}\left(u^{+} \partial_{x} u^{+}\right)\right) \pi_{>N}\left(u^{-} \partial_{x}^{m} u^{-}\right) \\
& =\int\left(\pi_{>N} \partial_{x}^{m}\left(u^{+} \partial_{x} u^{+}\right)\right) \pi_{>N}\left(u^{-} \partial_{x}^{m+1} u^{-}\right)-\int \partial_{x}^{m}\left(\pi_{>N}\left(u^{+} \partial_{x} u^{+}\right)\right) \pi_{>N} \partial_{x}\left(u^{-} \partial_{x}^{m} u^{-}\right) .
\end{aligned}
$$

By developing the derivative $\partial_{x}\left(u^{-} \partial_{x}^{m} u^{-}\right)=\partial_{x} u^{-} \partial_{x}^{m} u^{-}+u^{-} \partial_{x}^{m+1} u^{-}$and by replacing it in the last integral, we get

$$
\cdots=-\int \partial_{x}^{m}\left(\pi_{>N}\left(u^{+} \partial_{x} u^{+}\right)\right) \pi_{>N}\left(\partial_{x} u^{-} \partial_{x}^{m} u^{-}\right)
$$

By using integration by parts in the second integral we get

$$
\begin{aligned}
& \int\left(\pi_{>N} \partial_{x}^{m}\left(u^{-} \partial_{x} u^{-}\right)\right) \pi_{>N}\left(u^{+} \partial_{x}^{m+1} u^{+}\right)+\int \pi_{>N}\left(\partial_{x}^{m+1}\left(u^{-} \partial_{x} u^{-}\right)\right) \pi_{>N}\left(u^{+} \partial_{x}^{m} u^{+}\right) \\
& =-\int \pi_{>N}\left(\partial_{x}^{m}\left(u^{-} \partial_{x} u^{-}\right)\right) \pi_{>N}\left(\partial_{x} u^{+} \partial_{x}^{m} u^{+}\right)
\end{aligned}
$$

The proof follows by combining (9.13), (9.14), (9.15).

By a similar argument it is possible to prove that if $u(x)$ is as in Lemma 9.3 then the following identities occur: 


$$
\begin{aligned}
& \int H u\left(\partial_{x}^{m} \pi_{>N}\left(u \partial_{x} u\right)\right) \partial_{x}^{m+1} H u d x+\int H u\left(\partial_{x}^{m} u\right) \partial_{x}^{m+1}\left(\pi_{>N} H\left(u \partial_{x} u\right)\right) d x \\
& =-\int \partial_{x}^{m}\left(\pi_{>N}\left(u^{+} \partial_{x} u^{+}\right)\right) \pi_{>N}\left(\partial_{x} u^{-} \partial_{x}^{m} u^{-}\right) d x \\
& \quad-\int \partial_{x}^{m}\left(\pi_{>N}\left(u^{-} \partial_{x} u^{-}\right)\right) \pi_{>N}\left(\partial_{x} u^{+} \partial_{x}^{m} u^{+}\right) d x . \\
& \int H u\left(\partial_{x}^{m} \pi_{>N} H\left(u \partial_{x} u\right)\right) \partial_{x}^{m+1} u d x+\int H u\left(\partial_{x}^{m} H u\right) \partial_{x}^{m+1}\left(\pi_{>N}\left(u \partial_{x} u\right)\right) d x \\
& =-\int \partial_{x}^{m}\left(\pi_{>N}\left(u^{+} \partial_{x} u^{+}\right)\right) \pi_{>N}\left(\partial_{x} u^{-} \partial_{x}^{m} u^{-}\right) d x \\
& \quad-\int \partial_{x}^{m}\left(\pi_{>N}\left(u^{-} \partial_{x} u^{-}\right)\right) \pi_{>N}\left(\partial_{x} u^{+} \partial_{x}^{m} u^{+}\right) d x . \\
& \int u\left(\partial_{x}^{m} \pi_{>N}\left(u \partial_{x} u\right)\right) \partial_{x}^{m+1} u d x+\int u\left(\partial_{x}^{m} u\right) \partial_{x}^{m+1} \pi_{>N}\left(u \partial_{x} u\right) d x \\
& =-\int \partial_{x}^{m}\left(\pi_{>N}\left(u^{+} \partial_{x} u^{+}\right)\right) \pi_{>N}\left(\partial_{x} u^{-} \partial_{x}^{m} u^{-}\right) \\
& \quad-\int \partial_{x}^{m}\left(\pi_{>N}\left(u^{-} \partial_{x} u^{-}\right)\right) \pi_{>N}\left(\partial_{x} u^{+} \partial_{x}^{m} u^{+}\right) .
\end{aligned}
$$

\section{Some calculus inequalities}

Next we present some useful results related to the convergence of suitable numerical series.

Lemma 10.1. - The following estimate occurs :

$$
\sum_{\substack{|n+m|>N \\ 0<|n|,|m| \leq N}} \frac{1}{n^{2}} \frac{1}{|m|}=O\left(\frac{\ln N}{N}\right) \text { as } N \rightarrow \infty .
$$

Proof. - We have the identity

$$
\sum_{\substack{|n+m|>N \\ 0<|n|,|m| \leq N}} \frac{1}{n^{2}} \frac{1}{|m|}=2 \sum_{\substack{n+m>N \\ 0<n, m \leq N}} \frac{1}{n^{2}} \frac{1}{m}
$$

where we have used

$$
\begin{aligned}
\{(n, m) \in \mathbb{Z} \times \mathbb{Z}|0<| n|,| m \mid \leq N & |n+m|>N\} \\
=\{(n, m) \in \mathbb{Z} \times \mathbb{Z}|0<n, m \leq N,| n+m \mid>N\} & \\
& \cup\{(n, m) \in \mathbb{Z} \times \mathbb{Z}|-N \leq n, m<0,| n+m \mid>N\} .
\end{aligned}
$$

Next we continue the identity above

$$
\cdots=2 \sum_{0<n \leq N} \frac{1}{n^{2}}\left(\sum_{N-n<m \leq N} \frac{1}{m}\right) \leq 2 \sum_{0<n \leq N} \frac{1}{n^{2}} \frac{n}{N-n}=\frac{2}{N} \sum_{0<n \leq N}\left(\frac{1}{n}+\frac{1}{N-n}\right) .
$$

The proof follows since $\sum_{0<n \leq N} \frac{1}{n}=O(\ln N)$. 
Lemma 10.2. - The following estimate occurs:

$$
\sum_{\substack{|n+m+l|>N \\ 0<|n|,|m|,|l| \leq N}} \frac{1}{n^{2} m^{2}|l|}=O\left(\frac{\ln N}{N}\right) \text { as } N \rightarrow \infty .
$$

Proof. - We split the sum as follows:

$$
\sum_{\substack{|n+m+l|>N \\ 0<|n|,|m|,|l| \leq N}} \frac{1}{n^{2} m^{2}|l|} \leq \sum_{\substack{|n+l|>\frac{N}{2} \\ 0<|n|,|m|,|l| \leq N}} \frac{1}{n^{2} m^{2}|l|}+\sum_{\substack{|m|>\frac{N}{2} \\ 0<|n|,|m|,|l| \leq N}} \frac{1}{n^{2} m^{2}|l|}=I_{N}+I I_{N} .
$$

By using Lemma 10.1 we get

$$
I_{N}=O\left(\frac{\ln N}{N}\right)
$$

Concerning $I I_{N}$ we have

$$
I I_{N} \leq\left(\sum_{\frac{N}{2}<|m| \leq N} \frac{1}{m^{2}}\right)\left(\sum_{0<|l| \leq N} \frac{1}{|l|}\right)\left(\sum_{0<|n| \leq N} \frac{1}{|n|^{2}}\right) \leq C \frac{\ln N}{N} .
$$

\section{Proof of Theorem 1.2}

Along this section we shall write $\varphi_{N}(\omega)=\pi_{N}(\varphi(\omega)), \varphi_{N}^{ \pm}(\omega)=\pi_{ \pm}\left(\pi_{N} \varphi(\omega)\right.$ ) (where $\pi_{ \pm}$ are the projectors on the positive and negative frequencies) and

$$
\varphi(\omega)=\sum_{n \in \mathbb{Z} \backslash\{0\}} \frac{\varphi_{n}(\omega)}{|n|^{m+1}} e^{\mathrm{i} n x} .
$$

Moreover for any given $p(u) \in \cup_{n=1}^{\infty} \mathscr{P}_{n}(u)$ and $N \in \mathbb{N}, p_{N}^{*}(u)$ is defined in Section 8 .

Notice that due to the Hölder inequality the standard Gaussian variables $\left\{\varphi_{k}(\omega)\right\}_{k}$ satisfy:

$$
\forall q \in[1, \infty), k \in \mathbb{N} \exists C=C(k, q)>0 \text { s.t. } \sup _{j_{1}, \ldots, j_{k} \in \mathbb{Z} \backslash\{0\}}\left\|\varphi_{j_{1}} \cdots \varphi_{j_{k}}\right\|_{L_{\omega}^{q}} \leq C .
$$

This fact will be freely used in the sequel.

Lemma 11.1. - Let $m \geq 2$ be an integer and $p(u) \in \mathscr{P}_{3}(u)$ such that $\tilde{p}(u)=u \partial_{x}^{m} u \partial_{x}^{m+1} u$. Then for every $q \in[1, \infty)$ we have the following

$$
\lim _{N \rightarrow \infty}\left\|\int p_{N}^{*}\left(\pi_{N} u\right) d x\right\|_{L^{q}\left(d \mu_{m+1}\right)}=0 .
$$

Proof. - By using elementary properties of the Hilbert transform (i.e., $H^{2}=-\mathrm{Id}$, $\left.\int(H v) w d x=\int v(H w) d x\right)$ it is easy to check that if $p(u)$ is like in the assumptions then 
the quantities $\int p(u) d x$ can be always reduced to the following ones:

$$
\begin{aligned}
& \pm \int u \partial_{x}^{m} u \partial_{x}^{m+1} u d x, \pm \int(H u)\left(\partial_{x}^{m} H u\right)\left(\partial_{x}^{m+1} H u\right) d x \\
& \pm \int u\left(\partial_{x}^{m} H u\right)\left(\partial_{x}^{m+1} H u\right) d x, \pm \int(H u) \partial_{x}^{m} u \partial_{x}^{m+1} u d x \\
& \pm \int u \partial_{x}^{m} u\left(\partial_{x}^{m+1} H u\right) d x, \pm \int(H u)\left(\partial_{x}^{m} H u\right) \partial_{x}^{m+1} u d x \\
& \pm \int u\left(\partial_{x}^{m} H u\right)\left(\partial_{x}^{m+1} u\right) d x, \pm \int(H u)\left(\partial_{x}^{m} u\right)\left(\partial_{x}^{m+1} H u\right) d x
\end{aligned}
$$

First case: $p(u)=u \partial_{x}^{m} u \partial_{x}^{m+1} u$.

In this case we can write explicitly

$$
\left.p_{N}^{*}(u)=\pi_{>N}\left(u \partial_{x} u\right) \partial_{x}^{m} u \partial_{x}^{m+1} u+u \partial_{x}^{m}\left(\pi_{>N}\left(u \partial_{x} u\right)\right) \partial_{x}^{m+1} u+u \partial_{x}^{m} u \partial_{x}^{m+1}\left(\pi_{>N}\left(u \partial_{x} u\right)\right)\right) .
$$

Hence we get

$$
\int p_{N}^{*}\left(\pi_{N}(\varphi(\omega))\right) d x=I_{N}(\omega)+I I_{N}(\omega)
$$

where

$$
I_{N}(\omega)=\int \pi_{>N}\left(\varphi_{N}(\omega) \partial_{x}\left(\varphi_{N}(\omega)\right)\right) \partial_{x}^{m} \varphi_{N}(\omega) \partial_{x}^{m+1} \varphi_{N}(\omega) d x
$$

and

$$
\begin{aligned}
& I I_{N}(\omega)=\int \varphi_{N}(\omega)\left(\partial_{x}^{m} \pi_{>N}\left(\varphi_{N}(\omega)\left(\partial_{x} \varphi_{N}(\omega)\right)\right) \partial_{x}^{m+1} \varphi_{N}(\omega)\right) \\
&+\varphi_{N}(\omega)\left(\partial_{x}^{m} \varphi_{N}(\omega)\right) \partial_{x}^{m+1}\left(\pi_{>N}\left(\varphi_{N}(\omega) \partial_{x}\left(\varphi_{N}(\omega)\right)\right) d x\right.
\end{aligned}
$$

In order to estimate $I_{N}$ notice that

$$
\begin{aligned}
I_{N}(\omega) & =\int\left(\pi_{>N} \varphi_{N}(\omega) \partial_{x} \varphi_{N}(\omega)\right) \partial_{x}^{m} \varphi_{N}(\omega) \partial_{x}^{m+1} \varphi_{N}(\omega) d x \\
& =\sum_{\substack{0<\left|j_{1}\right|,\left|j_{2}\right|,\left|j_{3}\right|,\left|j_{4}\right| \leq N \\
\left|j_{1}+j_{2}\right|>N \\
j_{1}+j_{2}+j_{3}+j_{4}=0}} \frac{\varphi_{j_{1}}(\omega)}{\left|j_{1}\right|^{m+1}} \frac{\varphi_{j_{2}}(\omega)}{\left|j_{2}\right|^{m}} \frac{\varphi_{j_{3}}(\omega)}{\left|j_{3}\right|} \varphi_{j_{4}}(\omega) \\
y_{1} &
\end{aligned}
$$

and hence by the Minkowski inequality

$$
\begin{aligned}
\left\|I_{N}(\omega)\right\|_{L_{\omega}^{q}} \leq C \sum_{\substack{0<\left|j_{1}\right|,\left|j_{2}\right|,\left|j_{3}\right|,\left|j_{4}\right| \leq N \\
\left|j_{1}+j_{2}\right|>N \\
j_{1}+j_{2}+j_{3}+j_{4}=0}} \frac{1}{\left|j_{1}\right|^{m+1}\left|j_{2}\right|^{m}\left|j_{3}\right|} \\
\leq C\left(\sum_{0<\left|j_{3}\right| \leq N} \frac{1}{\left|j_{3}\right|}\right)\left(\sum_{\substack{0<\left|j_{1}\right|,\left|j_{2}\right| \leq N \\
\left|j_{1}+j_{2}\right|>N}} \frac{1}{\left|j_{1}\right|^{m+1}\left|j_{2}\right|^{m}}\right)=O\left(\frac{\ln ^{2} N}{N}\right)
\end{aligned}
$$

where we have used Lemma 10.1. 
Next we estimate $I I_{N}(\omega)$ (see (11.2)). Due to the identity (9.18) we are reduced to estimate the following quantities:

$$
\begin{aligned}
& I I_{N}^{\prime}(\omega)=-\int \partial_{x}^{m}\left(\pi_{>N}\left(\varphi_{N}^{+}(\omega) \partial_{x} \varphi_{N}^{+}(\omega)\right)\right) \pi_{>N}\left(\partial_{x} \varphi_{N}^{-}(\omega) \partial_{x}^{m} \varphi_{N}^{-}(\omega)\right) d x \\
& I I_{N}^{\prime \prime}(\omega)=-\int \partial_{x}^{m}\left(\pi_{>N}\left(\varphi_{N}^{-}(\omega) \partial_{x} \varphi_{N}^{-}(\omega)\right)\right) \pi_{>N}\left(\partial_{x} \varphi_{N}^{+}(\omega) \partial_{x}^{m} \varphi_{N}^{+}(\omega)\right) d x .
\end{aligned}
$$

Next we estimate $I I_{N}^{\prime}(\omega)$ (a similar argument works for $I I_{N}^{\prime \prime}(\omega)$ ). By the Leibnitz formula it is sufficient to prove that:

$$
\begin{gathered}
\left\|\int \pi_{>N}\left(\partial_{x}^{j} \varphi_{N}^{+}(\omega) \partial_{x}^{m-j+1} \varphi_{N}^{+}(\omega)\right) \pi_{>N}\left(\partial_{x} \varphi_{N}^{-}(\omega) \partial_{x}^{m} \varphi_{N}^{-}(\omega)\right) d x\right\|_{L_{\omega}^{q}}=o(1) \\
\text { as } N \rightarrow \infty \forall j=0,1, . ., m .
\end{gathered}
$$

Indeed the most delicate cases are $j=0, m$. All the other cases can be treated in the same way. In the case $j=0$ we are reduced to prove

$$
\lim _{N \rightarrow \infty}\left\|\int \pi_{>N}\left(\varphi_{N}^{+}(\omega) \partial_{x}^{m+1} \varphi_{N}^{+}(\omega)\right) \pi_{>N}\left(\partial_{x} \varphi_{N}^{-}(\omega) \partial_{x}^{m} \varphi_{N}^{-}(\omega)\right) d x\right\|_{L_{\omega}^{q}}=0 .
$$

For that purpose, we write

$$
\begin{aligned}
\limsup _{N \rightarrow \infty} \| & \sum_{\substack{0<\left|j_{1}\right|,\left|j_{2}\right|,\left|j_{3}\right|,\left|j_{4}\right| \leq N \\
j_{1}, j_{2}>0, j_{3}, j_{4}<0 \\
\left|j_{1}+j_{2}\right|>N \\
j_{1}+j_{2}+j_{3}+j_{4}=0}} \frac{\varphi_{j_{1}}(\omega)}{\left|j_{1}\right|^{m+1}} \varphi_{j_{2}}(\omega) \frac{\varphi_{j_{3}}(\omega)}{\left|j_{3}\right|^{m}} \frac{\varphi_{j_{4}}(\omega)}{\left|j_{4}\right|} \|_{L_{\omega}^{q}} \\
& \leq \limsup _{N \rightarrow \infty} C \sum_{\substack{0<\left|j_{1}\right|,\left|j_{3}\right|,\left|j_{4}\right| \leq N \\
\left|j_{3}+j_{4}\right|>N}} \frac{1}{\left|j_{1}\right|^{m+1}\left|j_{3}\right|^{m}\left|j_{4}\right|} \\
& \leq C \limsup _{N \rightarrow \infty}\left(\sum_{0<\left|j_{1}\right| \leq N} \frac{1}{\left|j_{1}\right|^{m+1}}\right) \sum_{\substack{0<\left|j_{3}\right|,\left|j_{4}\right| \leq N \\
\left|j_{3}+j_{4}\right|>N}} \frac{1}{\left|j_{3}\right|^{m}\left|j_{4}\right|}=O\left(\frac{\ln N}{N}\right)
\end{aligned}
$$

where we have used Lemma 10.1 at the last step.

To prove (11.3) for $j=m$ we have to show

$$
\lim _{N \rightarrow \infty}\left\|\int\left(\pi_{>N}\left(\partial_{x}^{m} \varphi_{N}^{+}(\omega) \partial_{x} \varphi_{N}^{+}(\omega)\right)\right) \pi_{>N}\left(\partial_{x} \varphi_{N}^{-}(\omega) \partial_{x}^{m} \varphi_{N}^{-}(\omega)\right)\right\|_{L_{\omega}^{q}}=0 .
$$

Indeed arguing as above (i.e., we replace the random vector $\varphi(\omega)$ by its random Fourier series and we apply the Minkowski inequality) we are reduced to prove that

$$
\lim _{N \rightarrow \infty} \sum_{\substack{0<\left|j_{1}\right|,\left|j_{2}\right|,\left|j_{3}\right|,\left|j_{2}\right| \leq N \\ j_{1}, j_{2}>0, j_{3}, j_{4}<0 \\\left|j_{1}+j_{2}\right|>N \\ j_{1}+j_{2}+j_{3}+j_{4}=0}} \frac{1}{\left|j_{1}\right|\left|j_{2}\right|^{m}\left|j_{3}\right|^{m}\left|j_{4}\right|}=0
$$

This estimate follows by combining the inequality

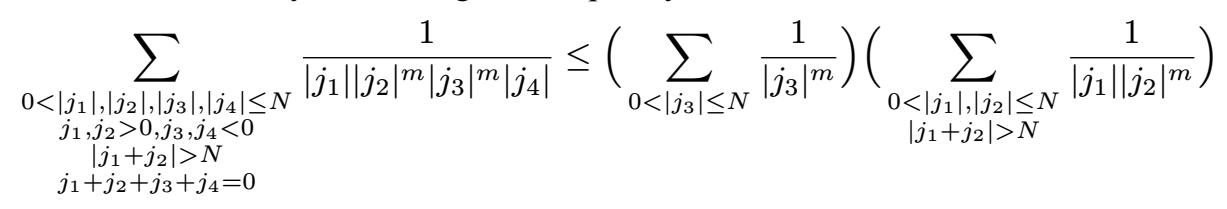


with Lemma 10.1.

Second case: $p(u)=u \partial_{x}^{m} H u \partial_{x}^{m+1} H u, H u \partial_{x}^{m} u \partial_{x}^{m+1} H u, H u \partial_{x}^{m} H u \partial_{x}^{m+1} u$.

All those cases can be treated as the previous one provided that we use (9.12), (9.16), (9.17) instead of (9.18) in the argument above.

Third case: $p(u)=u \partial_{x}^{m} u\left(\partial_{x}^{m+1} H u\right)$.

By definition we get:

$$
\int p_{N}^{*}\left(\pi_{N}(\varphi(\omega))\right) d x=I_{N}(\omega)+I I_{N}(\omega)+I I I_{N}(\omega)
$$

where

$$
\begin{aligned}
I_{N}(\omega) & =\int\left(\pi_{>N}\left(\varphi_{N}(\omega) \partial_{x} \varphi_{N}(\omega)\right)\right)\left(\partial_{x}^{m} \varphi_{N}(\omega)\right)\left(\partial_{x}^{m+1} H \varphi_{N}(\omega)\right) d x \\
I I_{N}(\omega) & =\int \varphi_{N}(\omega) \partial_{x}^{m}\left(\pi_{>N}\left(\varphi_{N}(\omega) \partial_{x} \varphi_{N}(\omega)\right)\right) \partial_{x}^{m+1} H \varphi_{N}(\omega) d x \\
I I I_{N}(\omega) & =\int \varphi_{N}(\omega) \partial_{x}^{m} \varphi_{N}(\omega) \partial_{x}^{m+1} H\left(\pi_{>N}\left(\varphi_{N}(\omega) \partial_{x} \varphi_{N}(\omega)\right)\right) d x .
\end{aligned}
$$

The term $I_{N}(\omega)$ can be estimated in the same way as (11.1) in the first case.

Concerning $I I_{N}(\omega)$ we use (9.6) and we get

$$
\begin{aligned}
I I_{N}(\omega)= & \sum_{j=1}^{m} c_{j}\left[\int \pi_{>N}\left(\partial_{x}^{j} \varphi_{N}^{+}(\omega) \partial_{x}^{m-j+1} \varphi_{N}^{+}(\omega)\right) \pi_{>N}\left(\varphi_{N}^{-}(\omega) \partial_{x}^{m+1} \varphi_{N}^{-}(\omega)\right)\right. \\
& \left.-\pi_{>N}\left(\partial_{x}^{j} \varphi_{N}^{-}(\omega) \partial_{x}^{m-j+1} \varphi_{N}^{-}(\omega)\right) \pi_{>N}\left(\varphi_{N}^{+}(\omega) \partial_{x}^{m+1} \varphi_{N}^{+}(\omega)\right)\right]
\end{aligned}
$$

Hence it is sufficient to show that

$$
\begin{gathered}
\limsup _{N \rightarrow \infty}\left\|\int \pi_{>N}\left(\partial_{x}^{j} \varphi_{N}^{+}(\omega) \partial_{x}^{m-j+1} \varphi_{N}^{+}(\omega)\right) \pi_{>N}\left(\varphi_{N}^{-}(\omega) \partial_{x}^{m+1} \varphi_{N}^{-}(\omega)\right)\right\|_{L_{\omega}^{q}}=0 \\
\forall j=1, \ldots, m .
\end{gathered}
$$

Indeed the most delicate cases are $j=1, m$ (that in turn can be treated in a similar way). First we focus on (11.4) for $j=m$. More precisely we have to prove

$$
\limsup _{N \rightarrow \infty}\left\|\int \pi_{>N}\left(\partial_{x}^{m} \varphi_{N}^{+}(\omega) \partial_{x} \varphi_{N}^{+}(\omega)\right) \pi_{>N}\left(\varphi_{N}^{-}(\omega) \partial_{x}^{m+1} \varphi_{N}^{-}(\omega)\right) d x\right\|_{L_{\omega}^{q}}=0 .
$$

By replacing the random vector $\varphi(\omega)$ by its Fourier randomized series we get:

$$
\begin{aligned}
& \int \pi_{>N}\left(\partial_{x}^{m} \varphi_{N}^{+}(\omega) \partial_{x} \varphi_{N}^{+}(\omega)\right) \pi_{>N}\left(\varphi_{N}^{-}(\omega) \partial_{x}^{m+1} \varphi_{N}^{-}(\omega)\right) d x \\
& =\sum_{\substack{0<\left|j_{1}\right|,\left|j_{2}\right|,\left|j_{3}\right|,\left|j_{4}\right| \leq N \\
j_{1}, j_{2}>0, j_{3}, j_{j}<0 \\
\left|j_{1}+j_{2}\right|>N \\
j_{1}+j_{2}+j_{3}+j_{4}=0}} \frac{\varphi_{j_{1}}(\omega)}{\left|j_{1}\right|} \frac{\varphi_{j_{2}}(\omega)}{\left|j_{2}\right|^{m}} \frac{\varphi_{j_{3}}(\omega)}{\left|j_{3}\right|^{m+1}} \varphi_{j_{4}}(\omega) .
\end{aligned}
$$

$4^{\text {e }}$ SÉRIE - TOME $46-2013-\mathrm{N}^{\circ} 2$ 
Hence by the Minkowski inequality we get:

$$
\begin{aligned}
& \limsup _{N \rightarrow \infty} \| \int \pi_{>N}\left(\partial_{x}^{m} \varphi_{N}^{+}(\omega) \partial_{x} \varphi_{N}^{+}(\omega)\right) \pi_{>N}\left(\varphi_{N}^{-}(\omega) \partial_{x}^{m+1} \varphi_{N}^{-}(\omega)\right) d x \|_{L_{\omega}^{q}} \\
& \leq C \limsup _{N \rightarrow \infty}\left(\sum_{0<\left|j_{3}\right| \leq N} \frac{1}{\left|j_{3}\right|^{m+1}}\right)\left(\sum_{\substack{0<\left|j_{1}\right|,\left|j_{2}\right|,\left|j_{3}\right| \leq N \\
\left|j_{1}+j_{2}\right|>N}} \frac{1}{\left|j_{1}\right|\left|j_{2}\right|^{m}}\right)=O\left(\frac{\ln N}{N}\right)
\end{aligned}
$$

where we have used Lemma 10.1 .

Concerning the estimate (11.4) for $j=1$ we can argue as above and we are reduced to prove that

$$
\lim _{N \rightarrow \infty} \sum_{\substack{0<\left|j_{1}\right|,\left|j_{2}\right|,\left|j_{3}\right|,\left|j_{4}\right| \leq N \\ j_{1}, j_{2}>0, j_{3}, j_{4}<0 \\\left|j_{1}+j_{2}\right|>N}} \frac{1}{\left|j_{1}\right|^{m}\left|j_{2}\right|\left|j_{3}\right|^{m+1}}=0
$$

that follows by Lemma 10.1. The estimate for $I I I_{N}(\omega)$ is similar to the one of $I I_{N}(\omega)$ provided that (9.7) is used instead of (9.6).

Fourth case: $p(u)=u\left(\partial_{x}^{m} H u\right)\left(\partial_{x}^{m+1} u\right), H u \partial_{x}^{m} H u \partial_{x}^{m+1} H u d x, H u \partial_{x}^{m} u \partial_{x}^{m+1} u$

They can be treated as in the third case provided that (9.1), (9.2) (resp. (9.8), (9.9) and (9.10),(9.11)) are used instead of (9.6) and (9.7).

Lemma 11.2. - Let $m \geq 2$ be an integer and $p(u) \in \mathscr{P}_{3}(u)$ such that $\tilde{p}(u)=\partial_{x}^{\alpha} u \partial_{x}^{\beta} u \partial_{x}^{\gamma} u$ with

$$
\alpha+\beta+\gamma=2 m+1,0 \leq \alpha \leq \beta \leq \gamma \text { and } \max \{\alpha, \beta, \gamma\} \leq m
$$

Then we have

$$
\lim _{N \rightarrow \infty}\left\|\int p_{N}^{*}\left(\pi_{N} u\right) d x\right\|_{L^{q}\left(d \mu_{m+1}\right)}=0, \forall q \in[1, \infty) .
$$

Proof. - We treat for simplicity the case $p=\partial_{x}^{\alpha} u \partial_{x}^{\beta} u \partial_{x}^{\gamma} u$ (the general case can be studied with a similar argument). Hence we get

$$
p_{N}^{*}\left(\varphi_{N}(\omega)\right)=I_{N}(\omega)+I I_{N}(\omega)+I I I_{N}(\omega)
$$

where

$$
\begin{aligned}
I_{N}(\omega) & =\int \partial_{x}^{\alpha}\left(\pi_{>N}\left(\varphi_{N}(\omega) \partial_{x} \varphi_{N}(\omega)\right)\right) \partial_{x}^{\beta} \varphi_{N}(\omega) \partial_{x}^{\gamma} \varphi_{N}(\omega) d x \\
I I_{N}(\omega) & =\int \partial_{x}^{\alpha} \varphi_{N}(\omega) \partial_{x}^{\beta}\left(\pi_{>N}\left(\varphi_{N}(\omega) \partial_{x} \varphi_{N}(\omega)\right)\right) \partial_{x}^{\gamma} \varphi_{N}(\omega) d x \\
I I I_{N}(\omega) & =\int \partial_{x}^{\alpha} \varphi_{N}(\omega) \partial_{x}^{\beta} \varphi_{N}(\omega) \partial_{x}^{\gamma}\left(\pi_{>N}\left(\varphi_{N}(\omega) \partial_{x} \varphi_{N}(\omega)\right)\right) d x
\end{aligned}
$$

We shall prove that

$$
\lim _{N \rightarrow \infty}\left\|I_{N}(\omega)\right\|_{L_{\omega}^{q}}=0
$$

(and in a similar way we can treat $I I_{N}(\omega)$ and $I I I_{N}(\omega)$ ). By the Leibnitz formula it is sufficient to prove

$$
\begin{gathered}
\lim _{N \rightarrow \infty}\left\|\int \pi_{>N}\left(\partial_{x}^{j} \varphi_{N}(\omega) \partial_{x}^{\alpha-j+1} \varphi_{N}(\omega)\right) \partial_{x}^{\beta} \varphi_{N}(\omega) \partial_{x}^{\gamma} \varphi_{N}(\omega) d x\right\|_{L_{\omega}^{q}}=0 \\
\forall j=0, \ldots, \alpha .
\end{gathered}
$$


We shall treat the case $j=0$ and all the other cases can be treated in a similar way. More precisely we shall prove that

$$
\lim _{N \rightarrow \infty}\left\|\int \pi_{>N}\left(\varphi_{N}(\omega) \partial_{x}^{\alpha+1} \varphi_{N}(\omega)\right) \partial_{x}^{\beta} \varphi_{N}(\omega) \partial_{x}^{\gamma} \varphi_{N}(\omega) d x\right\|_{L_{\omega}^{q}}=0 .
$$

Notice that we have

$$
\begin{aligned}
\int \pi_{>N}\left(\varphi_{N}(\omega) \partial_{x}^{\alpha+1} \varphi_{N}(\omega)\right) \partial_{x}^{\beta} \varphi_{N}(\omega) \partial_{x}^{\gamma} \varphi_{N}(\omega) d x & =\sum_{\substack{\left|j_{1}\right|,\left|j_{2}\right|,\left|j_{3}\right|,\left|j_{4}\right| \in(0, N] \\
\left|j_{1}+j_{2}\right|>N \\
j_{1}+j_{2}+j_{3}+j_{4}=0}} \frac{\varphi_{j_{1}}(\omega)}{\left|j_{1}\right|^{m+1}} \frac{\varphi_{j_{2}}(\omega)}{\left|j_{2}\right|^{m-\alpha}} \frac{\varphi_{j_{3}}(\omega)}{\left|j_{3}\right|^{m+1-\beta}} \frac{\varphi_{j_{4}}(\omega)}{\left|j_{4}\right|^{m+1-\gamma}}
\end{aligned}
$$

and hence by using the triangular inequality we get

$$
\begin{gathered}
\left\|\int \pi_{>N}\left(\varphi_{N}(\omega) \partial_{x}^{\alpha+1} \varphi_{N}(\omega)\right) \partial_{x}^{\beta} \varphi_{N}(\omega) \partial_{x}^{\gamma} \varphi_{N}(\omega) d x\right\|_{L_{\omega}^{q}} \\
\leq C \sum_{\substack{\left|j_{1}\right|,\left|j_{2}\right|,\left|j_{3}\right|,\left|j_{4}\right| \in(0, N] \\
\left|j_{1}+j_{2}\right|>N \\
j_{1}+j_{2}+j_{3}+j_{4}=0}} \frac{1}{\left|j_{1}\right|^{m+1}\left|j_{2}\right|^{m-\alpha}\left|j_{3}\right|^{m+1-\beta}\left|j_{4}\right|^{m+1-\gamma}}
\end{gathered}
$$

Next we consider three possible cases:

First subcase: $\alpha=1, \beta=\gamma=m$.

In this case we get

$$
\begin{aligned}
\| \int & \left(\pi_{>N} \varphi_{N}(\omega) \partial_{x}^{\alpha+1} \varphi_{N}(\omega)\right) \partial_{x}^{\beta} \varphi_{N}(\omega) \partial_{x}^{\gamma} \varphi_{N}(\omega) d x \|_{L_{\omega}^{q}} \\
& \leq C \sum_{\substack{\left|j_{1}\right|,\left|j_{2}\right|,\left|j_{4}\right| \in(0, N],\left|j_{1}+j_{2}\right|>N}} \frac{1}{\left|j_{1}\right|^{m+1}\left|j_{2}\right|^{m-1}\left|j_{4}\right|} \\
& \leq\left(\sum_{0<\left|j_{4}\right| \leq N} \frac{1}{\left|j_{4}\right|}\right)\left(\sum_{\substack{0<\left|j_{1}\right|,\left|j_{2}\right| \leq N,\left|j_{1}+j_{2}\right|>N}} \frac{1}{\left|j_{1}\right|^{m+1}\left|j_{2}\right|^{m-1}}\right)=O\left(\frac{\ln ^{2} N}{N}\right)
\end{aligned}
$$

where we have used Lemma 10.1.

Second subcase: $\alpha \leq \beta=\gamma<m$.

In this case we get

$$
\begin{aligned}
& \left\|\int\left(\pi_{>N} \varphi_{N}(\omega) \partial_{x}^{\alpha+1} \varphi_{N}(\omega)\right) \partial_{x}^{\beta} \varphi_{N}(\omega) \partial_{x}^{\gamma} \varphi_{N}(\omega) d x\right\|_{L_{\omega}^{q}} \\
& \quad \leq C \sum_{\substack{\left|j_{1}\right|,\left|j_{2}\right|,\left|j_{3}\right|,\left|j_{4}\right| \in(0, N] \\
\left|j_{1}+j_{2}\right|>N}} \frac{1}{\left|j_{1}\right|^{m+1}\left|j_{2}\right|\left|j_{3}\right|^{2}} \\
& \quad \leq C\left(\sum_{0<\left|j_{4}\right| \leq N} \frac{1}{\left|j_{3}\right|^{2}}\right)\left(\sum_{\substack{0<\left|j_{1}\right|,\left|j_{2}\right| \leq N,\left|j_{1}+j_{2}\right|>N}} \frac{1}{\left|j_{1}\right|^{m+1}\left|j_{2}\right|}\right)=O\left(\frac{\ln N}{N}\right)
\end{aligned}
$$

where we have used Lemma 10.1. 
Third subcase: $\alpha \leq \beta<\gamma \leq m$.

In this case we get

$$
\begin{aligned}
\left\|\int\left(\pi_{>N} \varphi_{N}(\omega) \partial_{x}^{\alpha+1} \varphi_{N}(\omega)\right) \partial_{x}^{\beta} \varphi_{N}(\omega) \partial_{x}^{\gamma} \varphi_{N}(\omega) d x\right\|_{L_{\omega}^{q}} & \leq C \sum_{\substack{\left|j_{1}\right|,\left|j_{2}\right|,\left|j_{3}\right|,\left|j_{4}\right| \in(0, N] \\
\left|j_{1}+j_{2}\right|>N}} \frac{1}{\left|j_{1}\right|^{m+1}\left|j_{2}\right|\left|j_{3}\right|^{2}}
\end{aligned}
$$

and we can conclude as in the previous case.

Lemma 11.3. - Let $p(u) \in \mathscr{P}_{4}(u)$ be such that $\tilde{p}(u)=\partial_{x}^{\alpha_{1}} u \partial_{x}^{\alpha_{2}} u \partial_{x}^{\alpha_{3}} u \partial_{x}^{\alpha_{4}} u$ with

$$
\alpha_{1} \leq \alpha_{2} \leq \alpha_{3} \leq \alpha_{4} \leq m \text { and } \alpha_{1}+\alpha_{2}+\alpha_{3}+\alpha_{4}=2 m \text {. }
$$

Then we have

$$
\lim _{N \rightarrow \infty}\left\|\int p_{N}^{*}\left(\pi_{N} u\right) d x\right\|_{L^{q}\left(d \mu_{m+1}\right)}=0, \forall q \in[1, \infty)
$$

Proof. - We shall treat the case $p=\partial_{x}^{\alpha_{1}} u \partial_{x}^{\alpha_{2}} u \partial_{x}^{\alpha_{3}} u \partial_{x}^{\alpha_{4}} u$. The general case follows in a similar way (indeed our argument will be essentially based on the Minkowski inequality and it is not affected in the case when $H$ appears in the expression of $p(u))$ ). Arguing as in Lemma 11.2 it is sufficient to prove that

$$
\lim _{N \rightarrow \infty}\left\|I_{N}\right\|_{L_{\omega}^{q}}=0, \lim _{N \rightarrow \infty}\left\|I I_{N}\right\|_{L_{\omega}^{q}}=0, \lim _{N \rightarrow \infty}\left\|I I I_{N}\right\|_{L_{\omega}^{q}}=0, \lim _{N \rightarrow \infty}\left\|I V_{N}\right\|_{L_{\omega}^{q}}=0
$$

where

$$
\begin{aligned}
I_{N} & =\int \partial_{x}^{\alpha_{1}} \pi_{>N}\left(\varphi_{N}(\omega) \partial_{x} \varphi_{N}(\omega)\right) \partial_{x}^{\alpha_{2}} \varphi_{N}(\omega) \partial_{x}^{\alpha_{3}} \varphi_{N}(\omega) \partial_{x}^{\alpha_{4}} \varphi_{N}(\omega) d x \\
I I_{N} & =\int \partial_{x}^{\alpha_{1}} \varphi_{N}(\omega) \partial_{x}^{\alpha_{2}} \pi_{>N}\left(\varphi_{N}(\omega) \partial_{x} \varphi_{N}(\omega)\right) \partial_{x}^{\alpha_{3}} \varphi_{N}(\omega) \partial_{x}^{\alpha_{4}} \varphi_{N}(\omega) d x \\
I I I_{N} & =\int \partial_{x}^{\alpha_{1}} \varphi_{N}(\omega) \partial_{x}^{\alpha_{2}} \varphi_{N}(\omega) \partial_{x}^{\alpha_{3}} \pi_{>N}\left(\varphi_{N}(\omega) \partial_{x} \varphi_{N}(\omega)\right) \partial_{x}^{\alpha_{4}} \varphi_{N}(\omega) d x \\
I V_{N} & =\int \partial_{x}^{\alpha_{1}} \varphi_{N}(\omega) \partial_{x}^{\alpha_{2}} \varphi_{N}(\omega) \partial_{x}^{\alpha_{3}} \varphi_{N}(\omega) \partial_{x}^{\alpha_{4}} \pi_{>N}\left(\varphi_{N}(\omega) \partial_{x} \varphi_{N}(\omega)\right) d x .
\end{aligned}
$$

We shall treat for simplicity only the term $I V_{N}$ (the other terms can be treated in a similar way). Hence we shall prove that $\lim _{N \rightarrow \infty}\left\|I V_{N}\right\|_{L_{\omega}^{q}}=0$. By the Leibniz rule it follows by the following estimates:

$$
\begin{gathered}
\lim _{N \rightarrow \infty}\left\|\int \partial_{x}^{\alpha_{1}} \varphi_{N}(\omega) \partial_{x}^{\alpha_{2}} \varphi_{N}(\omega) \partial_{x}^{\alpha_{3}} \varphi_{N}(\omega) \pi_{>N}\left(\partial_{x}^{j} \varphi_{N}(\omega) \partial_{x}^{\alpha_{4}-j+1} \varphi_{N}(\omega)\right) d x\right\|_{L_{\omega}^{q}}=0 \\
\forall j=0, \ldots, \alpha_{4} .
\end{gathered}
$$

We shall prove the estimate above for $j=0$ (all the other cases can be treated in a simpler way). Hence we have to show

$$
\lim _{N \rightarrow \infty}\left\|\int \partial_{x}^{\alpha_{1}} \varphi_{N}(\omega) \partial_{x}^{\alpha_{2}} \varphi_{N}(\omega) \partial_{x}^{\alpha_{3}} \varphi_{N}(\omega) \pi_{>N}\left(\varphi_{N}(\omega) \partial_{x}^{\alpha_{4}+1} \varphi_{N}(\omega)\right) d x\right\|_{L_{\omega}^{q}}=0 .
$$


Notice that

$$
\begin{aligned}
\int \partial_{x}^{\alpha_{1}} \varphi_{N}(\omega) \partial_{x}^{\alpha_{2}} \varphi_{N}(\omega) \partial_{x}^{\alpha_{3}} \varphi_{N}(\omega) \pi_{>N}\left(\varphi_{N}(\omega) \partial_{x}^{\alpha_{4}+1} \varphi_{N}(\omega)\right) d x \\
=\sum_{\substack{\left|j_{1}\right|,\left|j_{2}\right|,\left|j_{3}\right|,\left|j_{4}\right|,\left|j_{5}\right| \in(0, N],\left|j_{4}+j_{5}\right|>N \\
j_{1}+j_{2}+j_{3}+j_{4}+j_{5}=0}} \frac{\varphi_{j_{1}}(\omega)}{\left|j_{1}\right|^{m+1-\alpha_{1}}} \frac{\varphi_{j_{2}}(\omega)}{\left|j_{2}\right|^{m+1-\alpha_{2}}} \frac{\varphi_{j_{3}}(\omega)}{\left|j_{3}\right|^{m+1-\alpha_{3}}} \frac{\varphi_{j_{4}}(\omega)}{\left|j_{4}\right|^{m+1}} \frac{\varphi_{j_{5}}(\omega)}{\left|j_{5}\right|^{m-\alpha_{4}}}
\end{aligned}
$$

and hence by the Minkowski inequality and Lemma 10.2 we get

$$
\begin{aligned}
\left\|\int \partial_{x}^{\alpha_{1}} \varphi_{N} \partial_{x}^{\alpha_{2}} \varphi_{N} \partial_{x}^{\alpha_{3}} \varphi_{N} \pi_{>N}\left(\varphi_{N} \partial_{x}^{\alpha_{4}+1} \varphi_{N}\right) d x\right\|_{L_{\omega}^{q}} \\
\qquad C \sum_{\substack{\left|j_{1}\right|,\left|j_{2}\right|,\left|j_{3}\right|,\left|j_{4}\right|,\left|j_{5}\right| \in(0, N],\left|j_{1}+j_{2}+j_{3}\right|>N}} \frac{1}{\left|j_{1}\right|^{2}\left|j_{2}\right|^{2}\left|j_{3}\right|\left|j_{4}\right|}=O\left(\frac{\ln ^{2} N}{N}\right)
\end{aligned}
$$

where we have used the fact that by assumption necessarily $\alpha_{1}, \alpha_{2}<m$.

Lemma 11.4. - Let $p_{j}(u) \in \mathscr{P}_{j}(u)$ with $j \geq 5$ be such that $\tilde{p}_{j}(u)=\prod_{k=1}^{j} \partial_{x}^{\alpha_{k}} u$ where

$$
\alpha_{1} \leq \cdots \leq \alpha_{j} \leq m \text { and } \sum_{k=1}^{j} \alpha_{k} \leq 2 m-1 \text {. }
$$

Then

$$
\lim _{N \rightarrow \infty}\left\|\int p_{N}^{*}\left(\pi_{N} u\right) d x\right\|_{L^{q}\left(d \mu_{m+1}\right)}=0, \forall q \in[1, \infty) .
$$

Proof. - It follows as Lemma 11.3.

Proof of Theorem 1.2. - It follows by combining Lemma 11.1, 11.2, 11.3, 11.4 with Proposition 8.3.

\section{Acknowledgments}

We are grateful to the referee for the valuable remarks and to Farinaz Wigmans for improving the presentation of the paper. The first author is partially supported by a grant from the European Research Council.

\section{REFERENCES}

[1] L. Abdelouhab, J. L. Bona, M. Felland, J.-C. Saut, Nonlocal models for nonlinear, dispersive waves, Phys. D 40 (1989), 360-392.

[2] J. Bourgain, Periodic nonlinear Schrödinger equation and invariant measures, Comm. Math. Phys. 166 (1994), 1-26.

[3] J. Bourgain, Invariant measures for the 2D-defocusing nonlinear Schrödinger equation, Comm. Math. Phys. 176 (1996), 421-445.

[4] D. C. BRydges, G. Slade, Statistical mechanics of the 2-dimensional focusing nonlinear Schrödinger equation, Comm. Math. Phys. 182 (1996), 485-504. 
[5] N. Burq, F. Planchon, On well-posedness for the Benjamin-Ono equation, Math. Ann. 340 (2008), 497-542.

[6] N. Burq, L. Thomann, N. Tzvetkov, Long time dynamics for the one dimensional non linear Schrödinger equation, to appear in Ann. Inst. Fourier.

[7] A. D. Ionescu, C. E. Kenig, Global well-posedness of the Benjamin-Ono equation in low-regularity spaces, J. Amer. Math. Soc. 20 (2007), 753-798.

[8] J. L. Lebowitz, H. A. Rose, E. R. Speer, Statistical mechanics of the nonlinear Schrödinger equation, J. Statist. Phys. 50 (1988), 657-687.

[9] M. Ledoux, M. Talagrand, Probability in Banach spaces, Ergebn. Math. Grenzg. 23, Springer, 1991.

[10] Y. Matsuno, Bilinear transformation method, Mathematics in Science and Engineering 174, Academic Press Inc., 1984.

[11] L. Molinet, Global well-posedness in $L^{2}$ for the periodic Benjamin-Ono equation, Amer. J. Math. 130 (2008), 635-683.

[12] A. R. Nahmod, T. Oh, L. Rey-Bellet, G. Staffilani, Invariant weighted Wiener measures and almost sure global well-posedness for the periodic derivative NLS, J. Eur. Math. Soc. (JEMS) 14 (2012), 1275-1330.

[13] T. TAO, Global well-posedness of the Benjamin-Ono equation in $H^{1}(\mathbf{R})$, J. Hyperbolic Differ. Equ. 1 (2004), 27-49.

[14] N. Tzvetkov, Construction of a Gibbs measure associated to the periodic BenjaminOno equation, Probab. Theory Related Fields 146 (2010), 481-514.

[15] P. E. Zhidkov, Korteweg-de Vries and nonlinear Schrödinger equations: qualitative theory, Lecture Notes in Math. 1756, Springer, 2001.

(Manuscrit reçu le 4 octobre 2011; accepté, après révision, le 25 juin 2012.)

\footnotetext{
Nikolay Tzvetrov

Département de Mathématiques

Université de Cergy-Pontoise

2, avenue Adolphe Chauvin

95302 Cergy-Pontoise Cedex, France and

Institut Universitaire de France

E-mail: nikolay.tzvetkov@u-cergy.fr

Nicola Visciglia Università Degli Studi di Pisa

Dipartimento di Matematica "L. Tonelli"

Largo Bruno Pontecorvo 5 I

56127 Pisa, Italy

E-mail: viscigli@dm.unipi.it
} 